\title{
A PARTITION CALCULUS IN SET THEORY
}

\section{P. ERDÖS AND R. RADO}

1. Introduction. Dedekind's pigeon-hole principle, also known as the box argument or the chest of drawers argument (Schubfachprinzip) can be described, rather vaguely, as follows. If sufficiently many objects are distributed over not too many classes, then at least one class contains many of these objects. In $1930 \mathrm{~F}$. P. Ramsey [12] discovered a remarkable extension of this principle which, in its simplest form, can be stated as follows. Let $S$ be the set of all positive integers and suppose that all unordered pairs of distinct elements of $S$ are distributed over two classes. Then there exists an infinite subset $A$ of $S$ such that all pairs of elements of $A$ belong to the same class. As is well known, Dedekind's principle is the central step in many investigations. Similarly, Ramsey's theorem has proved itself a useful and versatile tool in mathematical arguments of most diverse character. The object of the present paper is to investigate a number of analogues and extensions of Ramsey's theorem. We shall replace the sets $S$ and $A$ by sets of a more general kind and the unordered pairs, as is the case already in the theorem proved by Ramsey, by systems of any fixed number $r$ of elements of $S$. Instead of an unordered set $S$ we consider an ordered set of a given order type, and we stipulate that the set $A$ is to be of a prescribed order type. Instead of two classes we admit any finite or infinite number of classes. Further extension will be explained in $\S \S 2,8$ and 9.

The investigation centres round what we call partition relations connecting given cardinal numbers or order types and in each given case the problem arises of deciding whether a particular partition relation is true or false. It appears that a large number of seemingly unrelated arguments in set theory are, in fact, concerned with just such a problem. It might therefore be of interest to study such relations for their own sake and to build up a partition calculus which might serve as a new and unifying principle in set theory.

In some cases we have been able to find best possible partition relations, in one sense or another. In other cases the methods available to the authors do not seem to lead anywhere near the ultimate

Part of this paper was material from an address delivered by P. Erdös under the title Combinatorial problems in set theory before the New York meeting of the Society on October 24, 1953, by invitation of the Committee to Select Hour Speakers for Eastern Sectional Meetings; received by the editors May 17, 1955. 
truth. The actual description of results must be deferred until the notation and terminology have been given in detail. The most concrete results are perhaps those given in Theorems $25,31,39$ and 43. Of the unsolved problems in this field we only mention the following question. Is the relation $\lambda \rightarrow\left(\omega_{0} 2, \omega_{0} 2\right)^{2}$ true or false? Here, $\lambda$ denotes the order type of the linear continuum.

The classical, Cantorian, set theory will be employed throughout. In some arguments it will be advantageous to assume the continuum hypothesis $2^{\aleph_{0}}=\boldsymbol{N}_{1}$ or to make some even more general assumption. In every such case these assumptions will be stated explicitly.

The authors wish to thank the referee for many valuable suggestions and for having pointed out some inaccuracies.

2. Notation and definitions. Capital letters, except $\Delta$, denote sets, small Greek letters, except possibly $\pi$, order types, briefly: types, and $k, l, m, n, \kappa, \lambda, \mu, \nu$ denote ordinal numbers (ordinals). The letters $r, s$ denote non-negative integers, and $a, b, d$ cardinal numbers (cardinals). No distinction will be made between finite ordinals and the corresponding finite cardinals. Union and intersection of $A$ and $B$ are $A+B$ and $A B$ respectively, and $A \subset B$ denotes inclusion, in the wide sense. For any $A$ and $B, A-B$ is the set of all $x \in A$ such that $x \in B$. No confusion will arise from our using 0 to denote both zero and the empty set. If $p(x)$ is a proposition involving the general element $x$ of a set $A$ then $\{x: p(x)\}$ is the set of all $x \in A$ such that $p(x)$ is true.

$\eta$ and $\lambda$ are the types, under order by magnitude, of the set of all rational and of all real numbers respectively. $\lambda$ will also be used freely as a variable ordinal in places where no confusion can arise. The relation $\alpha \leqq \beta$ means that every set, ordered according to $\beta$, contains a subset of type $\alpha$, and $\alpha \geqq \beta$ is the negation of $\alpha \leqq \beta$. To every type $\alpha$ there belongs the converse type $\alpha^{*}$ obtained from $\alpha$ by replacing every order relation $x<y$ by the corresponding relation $x>y$. We put

$$
[m, n]=\{\nu: m \leqq \nu<n\} \quad \text { for } m \leqq n .
$$

The symbol

$$
\left\{x_{0}, x_{1}, \cdots\right\}<
$$

denotes the set $\left\{x_{0}, x_{1}, \cdots\right\}$ and, at the same time, expresses the fact that $x_{0}<x_{1}<\cdots$. Brackets \{\} are only used in order to define sets by means of a list of their elements. For typographical convenience we write

$$
\sum[x \in A] f(x)
$$


instead of $\sum_{x \in A} f(x)$, and we proceed similarly in the case of products etc. or when the condition $x \in A$ is replaced by some other type of condition.

The cardinal of $S$ is $|S|$, and the cardinal of $\alpha$ is $|\alpha|$. For every cardinal $a$, the symbol $a^{+}$denotes the next larger cardinal. If $a=b^{+}$ for some $b$, then we put $a^{-}=b$, and if $a$ is not of the form $b^{+}$, i.e. if $a$ is zero or a limit cardinal, then we put $a^{-}=a$. Similarly, we put $k^{-}=l$, if $k=l+1$, and $k^{-}=k$, if $k=0$ or if $k$ is a limit ordinal. If $S$ is ordered by means of the order relation $x<y$, then the type of $S$ is denoted by $\bar{S}_{<}$and, if no confusion can arise, by $\bar{S}$. For any $a>1$ we denote by $a^{\prime}$ the least cardinal $|n|$ such that $a$ can be represented in the form $\sum[\nu<n] a_{\nu}$ where $a_{\nu}<a$ for all $\nu<n$. This cardinal $a^{\prime}$, the cofinality cardinal belonging to $a$, is closely related to the cofinality ordinal $c f(\beta)$ of an ordinal $\beta$ introduced by Tarski [17]. A regular cardinal is a cardinal $a$ such that $a^{\prime}=a$. The least ordinal of a given cardinal $a$ is the initial ordinal belonging to $a$. Initial ordinals are the finite ordinals and the infinite ordinals $\omega_{\nu}$ of cardinal $\boldsymbol{\aleph}_{\nu}$. We put

$$
[S]^{a}=\{X: X \subset S ;|X|=a\} .
$$

In particular, $[S]^{a}=0$ if $|S|<a$. The relation

$$
A=\sum^{\prime}[\nu<k] A_{\nu}=A_{0}+{ }^{\prime} A_{1}+{ }^{\prime} \ldots
$$

means, by definition, that $A=\sum[\nu<k] A_{\nu}$ and, also,

$$
A_{\mu} A_{\nu}=0 \quad(\mu<\nu<k) .
$$

Fundamental throughout this paper is the partition relation

$$
a \rightarrow(b, d)^{2}
$$

introduced in [6]. More generally, for any $a, b_{\nu}, k, r$ the relation

$$
a \rightarrow\left(b_{0}, b_{1}, \cdots\right)_{k}^{r}
$$

is said to hold if, and only if, the following statement is true. The cardinals $b_{\nu}$ are defined for $\nu<k$. Whenever

$$
|S|=a ; \quad[S]^{r}=\sum[\nu<k] K_{\nu},
$$

then there are $B C S ; \nu<k$ such that

$$
|B|=b_{\nu} ; \quad[B]^{r} \subset K_{\nu} .
$$

For $k<\omega_{0}$ we also write (1) in the form

$$
a \rightarrow\left(b_{0}, b_{1}, \cdots, b_{k-1}\right)^{r},
$$


and if $k$ is arbitrary, and $b_{\nu}=b$ for all $\nu<k$, then we may write (1) in the form

$$
a \rightarrow(b)_{k}^{r} \text {. }
$$

We also introduce partition relations between types. By definition, the relation

$$
\alpha \rightarrow\left(\beta_{0}, \beta_{1}, \cdots\right)_{k}^{r}
$$

holds if, and only if, $\beta_{\nu}$ is defined for $\nu<k$ and if, whenever a set $S$ is ordered and

$$
\bar{S}=\alpha ; \quad[S]^{r}=\sum[\nu<k] K_{\nu},
$$

there are $B \subset S ; \nu<k$ such that

$$
\bar{B}=\beta_{\nu} ; \quad[B] r \subset K_{\nu} .
$$

If $k<\omega_{0}$, or if all $\beta_{v}$ are equal to each other, we use an alternative notation for (2) analogous to that relating to (1). The negation of (1), and similarly in the case of (2), is denoted by

$$
a \leftrightarrow\left(b_{0}, b_{1}, \cdots\right)_{k}^{r} .
$$

We mention in passing that the gulf between (1) and (2) can be bridged by the introduction of more general partition relations referring to partial orders. These will, however, not be considered here.

If $a \geqq \boldsymbol{\aleph}_{0}$ then, clearly, $a^{\prime}$ is the least cardinal $\boldsymbol{\aleph}_{n}$ such that $a \rightarrow(a)_{\omega_{n}}^{\prime}$. Also, $\boldsymbol{\aleph}_{m}$ is regular if, and only if, $\boldsymbol{\aleph}_{m} \rightarrow\left(\boldsymbol{\aleph}_{m}\right)_{\omega_{n}}^{1}$ for all $n<m$. Finally, the relation $a \rightarrow\left(b_{0}^{+}, b_{1}^{+}, \cdots\right)_{k}^{1}$ is equivalent to $\sum[\nu<k] b_{\nu}<a$.

We now introduce some abbreviations. Let $S$ be ordered. Then, for $x \in S$,

$$
L(x)=\left\{y:\{y, x\}_{<} \subset S\right\} ; \quad R(x)=\{y:\{x, y\}<\subset S\} .
$$

If, in addition, $[S]^{r}=\sum[\nu<k] K_{\nu}$, then, for $B \subset S ; \nu<k$,

$$
\begin{aligned}
F_{\nu}(B) & =\left\{\bar{A}: A \subset B ;[A]^{r} \subset K_{\nu}\right\}, \\
{\left[K_{\nu}\right] } & =F_{\nu}(S) .
\end{aligned}
$$

In the special case $r=2$, we put, for $x \in S ; \nu<k, L_{\nu}(x)=\left\{y:\{y, x\}_{<}\right.$ $\left.\in K_{\nu}\right\} ; R_{\nu}(x)=\left\{y:\{x, y\}_{<} \in K_{\nu}\right\} ; U_{\nu}=L_{\nu}+R_{\nu} . U_{\nu}$ is independent of the order of $S$. If $0 \neq A \subset S$, and if $W(x)$ is any one of the functions $L, R, L_{\nu}, R_{\nu}, U_{\nu}$, then we put

$$
W(A)=\prod[x \in A] W(x) .
$$

Also, $W(0)=S$. If $n<\omega_{0}$, then we write $W\left(x_{0}, \cdots, x_{n-1}\right)$ instead of 
$W\left(\left\{x_{0}, \cdots, x_{n-1}\right\}\right)$. It will always be clear from the context to which ordered set $S$ and to which partition of $[S]^{r}$ these functions refer. We shall occasionally make use of the notation and the calculus of partitions (distributions) summarized in [5, p. 419]. The meaning of canonical partition relations

$$
\alpha \rightarrow *(\beta)^{r}
$$

and that of polarized partition relations

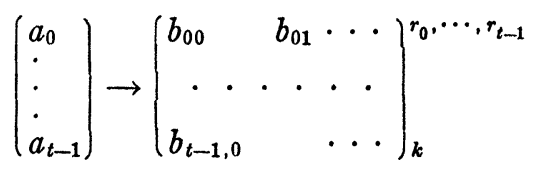

will be given in $\S \S 8$ and 9 respectively. The relation $\alpha \rightarrow(\beta)_{d}^{r}$ will be defined in $\$ 4$.

\section{Previous results.}

THeorem 1. If $k<\omega_{0}$ then $\boldsymbol{\aleph}_{0} \rightarrow\left(\boldsymbol{\aleph}_{0}\right)_{k}^{r}[12$, Theorem A ].

THEOREM 2. If $k, n<\omega_{0}$, then, for some $f=f(k, n, r)<\boldsymbol{\aleph}_{0}$,

$$
f \rightarrow(n)_{k}^{r}
$$

$[12$, Theorem B $]$.

Theorem 3. (i) If $a \geqq \boldsymbol{\aleph}_{0}$, then $a \rightarrow\left(\boldsymbol{\aleph}_{0}, a\right)^{2}$.

(ii) $\boldsymbol{\aleph} \omega_{0} \rightarrow\left(\boldsymbol{\aleph}_{1}, \boldsymbol{\aleph} \omega_{0}\right)^{2}$.

(i) is proved in $[2,5.22]$. This formula will be restated and proved as Theorem 44.

(ii) is in $[3$, p. 366] and will follow from Theorem 36 (iv).

TheOREM 4. (i) If $a \geqq \boldsymbol{\aleph}_{0}$, then $\left(a^{a}\right)^{+} \rightarrow\left(a^{+}\right)_{a}^{2}$.

(ii) If $a \geqq \boldsymbol{\aleph}_{0}$, then $a^{a} \rightarrow(3)_{a}^{2}$.

(iii) If $2^{\boldsymbol{\aleph}_{n}}=\boldsymbol{\aleph}_{n+1}$, then $\boldsymbol{\aleph}_{n+2} \rightarrow\left(\boldsymbol{\aleph}_{n+1}, \boldsymbol{\aleph}_{n+2}\right)^{2}$.

(i) is given in [3] and will be deduced as a corollary of Theorem 39 .

(ii) is in [3, p. 364], and (iii) is [3, Theorem II] and follows from Theorem $7(\mathrm{i}) .^{1}$

Theorem 5. If $\phi \leqq \lambda ;|\phi|>\boldsymbol{\aleph}_{0}$, then, for $\alpha<\omega_{0} 2 ; \beta<\omega_{0}^{2} ; \gamma<\omega_{1}$,

(i) $\phi \rightarrow\left(\omega_{0}, \gamma\right)^{2}$.

(ii) $\phi \rightarrow(\alpha, \beta)^{2}$.

1 The partition relations occurring in (i) and (ii) are to be interpreted in the obvious way. Their formal definition is given in $\$ 4$. 
(i) is [5, Theorem 5], and (ii) is [5, Theorem 7]. Both results will follow from Theorem 31 .

THEOREM 6. $\eta \rightarrow\left(\boldsymbol{\aleph}_{0}, \eta\right)^{2}$.

This relation, a cross between (1) and (2), has, by definition, the following meaning. If $\bar{S}=\eta ;[S]^{2}=K_{0}+K_{1}$, then there is $A \subset S$ such that either

$$
|A|=\aleph_{0} ;[A]^{2} \subset K_{0}
$$

or

$$
\bar{A}=\eta ;[A]^{2} \subset K_{1} \text {. }
$$

This result is $[5$, Theorem 4$]$.

THEOREM 7. If $a \geqq \aleph_{0}$, and if $b$ is minimal such that $a^{b}>a$, then

(i) $a^{+} \rightarrow\left(b, a^{+}\right)^{2}$

(ii) $a^{b} \rightarrow\left(b^{+}, a^{+}\right)^{2}$.

These results are contained in $[6$, p. 437]. (i) will follow from Theorem $34 .^{2}$

THEOREM 8. If $2^{\boldsymbol{N}_{\nu}}=\boldsymbol{\aleph}_{\nu+1}$ for all $\nu$, and if $a$ is a regular limit number ${ }^{3}$ then, for every $b<a, a \rightarrow(b, a)^{2}$.

This result is $[6$, Lemma 3$]$, and will follow from Theorem 34 .

Theorem 9. If $\phi \leqq \lambda ;|\phi|=|\lambda|$, then $\lambda \rightarrow(\phi, \phi)^{1}$.

This result is due to Sierpiński who kindly communicated it to one of us. It will follow from Theorem 29. Our proof of Theorem 29 uses some of Sierpiński's ideas.

Theorem 10. For any $a, a \rightarrow\left(\boldsymbol{\aleph}_{0}, \boldsymbol{\aleph}_{0}\right) \boldsymbol{\aleph}_{0}$.

This is in $[5$, p. 434$]$. The last result justifies our restriction to the case of finite "exponents" $r$.

\section{Simple properties of partition relations.}

THEOREM 11. The two relations
(i) $\alpha \rightarrow\left(\beta_{0}, \beta_{1}, \cdots\right)_{k}^{r}$
(ii) $\alpha^{*} \rightarrow\left(\beta_{0}^{*}, \beta_{1}^{*}, \cdots\right)_{k}^{r}$

are equivalent.

2 By methods similar to those used in [17] one can show that (i) $b \leqq a^{\prime}$ for all $a>1$, (ii) $b=a^{\prime}$ for those $a>1$ for which $d<a$ implies $2^{d} \leqq a$.

${ }^{3}$ It is not known if regular limit numbers $>\boldsymbol{N}_{0}$ exist or not. Cf. [13, p. 224]. 
Proof. Let (i) hold; $\bar{S}_{<}=\alpha^{*} ;[S]^{r}=\sum[\nu<k] K_{\nu}$. Then $\bar{S}_{>}=\alpha$. Hence, by hypothesis, there are $A C S ; \nu<k$ such that $\bar{A}_{>}=\beta_{\nu}$; $[A]^{r} \subset K_{\nu}$. Then $\bar{A}_{<}=\beta_{\nu}^{*}$. This proves (ii), and the theorem follows by reasons of symmetry.

TheOREM 12. Let $\alpha \rightarrow\left(\beta_{0}, \beta_{1}, \cdots\right)_{k}^{r} ; \alpha \leqq \alpha^{(1)} ; k \geqq k^{(1)}$;

Then

$$
\begin{array}{lr}
\beta_{\nu} \geqq \beta_{\nu}^{(1)} & \left(\nu<k^{(1)}\right), \\
\beta_{\nu} \geqq r & \left(k^{(1)} \leqq \nu<k\right) .
\end{array}
$$

$$
\alpha^{(1)} \rightarrow\left(\beta_{0}^{(1)}, \beta_{1}^{(1)}, \cdots\right)_{k}^{r(1)} .
$$

An analogous result holds when the types $\alpha, \beta_{\nu}$ are replaced by cardinals.

PROof. It suffices to consider the case of types. Let

$$
\bar{S}^{(1)}=\alpha^{(1)} ; \quad\left[S^{(1)}\right]^{r}=\sum\left[\nu<k^{(1)}\right] K_{\nu}^{(1)} .
$$

Then there is $S \subset S^{(1)}$ such that $\bar{S}=\alpha$. Then

$$
[S]^{r}=\sum[\nu<k] K_{\nu},
$$

where $K_{\nu}=K_{\nu}^{(1)}[S]^{r}$ for $\nu<k^{(1)}$, and $K_{\nu}=0$ otherwise. By hypothesis, there are $A \subset S ; \nu<k$ such that

$$
\bar{A}=\beta_{\nu} ; \quad[A]^{r} \subset K_{\nu} .
$$

If $\nu \geqq k^{(1)}$, then $|A|=\left|\beta_{\nu}\right| \geqq r ; 0 \neq[A]^{r} \subset K_{\nu}$ which is a contradiction. Hence $\nu<k^{(1)}$. There is $A^{(1)} \subset A$ such that $\bar{A}^{(1)}=\beta_{\nu}^{(1)}$. Then $\left[A^{(1)}\right]^{r}$ $\subset[A]^{r} \subset K_{\nu} \subset K_{\nu}^{(1)}$, and the assertion follows.

THEOREM 13. If $\alpha \rightarrow\left(\beta_{0}, \beta_{1}, \cdots\right)_{k}^{r}$ then

$$
|\alpha| \rightarrow\left(\left|\beta_{0}\right|,\left|\beta_{1}\right|, \cdots\right)_{k}^{r} \text {. }
$$

Proof. Let $|S|=|\alpha| ;[S]^{r}=\sum[\nu<k] K_{\nu}$. We order $S$ so that $\bar{S}=\alpha$. Then there are $A \subset S ; \nu<k$ such that $\bar{A}=\beta_{\nu} ;[A]^{r} \subset K_{\nu}$. Then $|A|=\left|\beta_{\nu}\right|$, and the theorem follows.

THEOREM 14. If $\beta_{\nu}$ is an initial ordinal, for all $\nu<k$, then the two relations

$$
\begin{aligned}
m & \rightarrow\left(\beta_{0}, \beta_{1}, \cdots\right)_{k}^{r}, \\
|m| & \rightarrow\left(\left|\beta_{0}\right|,\left|\beta_{1}\right|, \cdots\right)_{k}^{r}
\end{aligned}
$$

are equivalent.

Proof. By Theorem 13, (3) implies (4). Now suppose that (4) 
holds. Let $\bar{S}=m ;[S]^{r}=\sum[\nu<k] K_{\nu}$. Then $|S|=|m|$, and hence, by (4), there are $A \subset S ; \nu<k$ such that $|A|=\left|\beta_{\nu}\right| ;[A]^{r} \subset K_{\nu}$. Then, as $\beta_{\nu}$ is an initial ordinal, $\bar{A} \geqq \beta_{\nu}$, and there is $B \subset A$ such that $\bar{B}=\beta_{\nu}$. This proves (3).

TheOREM 15. If $1+\alpha \rightarrow\left(1+\beta_{0}, 1+\beta_{1}, \cdots\right)_{k}^{1+r}$, then

$$
\alpha \rightarrow\left(\beta_{0}, \beta_{1}, \cdots\right)_{k}^{r} \text {. }
$$

In this proposition, $1+\alpha$ and $1+\beta_{\nu}$ may be replaced by $\alpha+1$ and $\beta_{\nu}+1$ respectively. Also, the types $\alpha, \beta_{\nu}$ may be replaced by cardinals.

Proof. Let $\bar{S}=\alpha$. Let $x_{0}$ be an object which is not an element of $S$, and put $S_{0}=S+\left\{x_{0}\right\}$. The order of $S$ is extended to an order of $S_{0}$ by stipulating that $x_{0} \in L(S)$. Then $\bar{S}_{0}=1+\alpha$. Now let $[S]^{r}$ $=\sum[\nu<k] K_{\nu}$. Then $\left[S_{0}\right]{ }^{1+r}=\sum[\nu<k] K_{0 \nu}$, where $K_{0 \nu}=\left\{\left\{y_{0}, \cdots\right.\right.$, $\left.\left.y_{r}\right\}_{<:}\left\{y_{1}, \cdots, y_{r}\right\} \in K_{v}\right\}$. If $1+\alpha \rightarrow\left(1+\beta_{0}, 1+\beta_{1}, \cdots\right)_{k}^{1+r}$, then there are $A_{0} \subset S_{0} ; \nu<k$ such that $\bar{A}_{0}=1+\beta_{\nu} ;\left[A_{0}\right]^{1+r} \subset K_{0 v}$. Then $A_{0}=\left\{y_{0}\right\}+A ; y_{0} \in L(A)$;

$$
\bar{A}=\beta_{\nu} ; \quad[A]^{r} \subset K_{\nu} .
$$

This proves the first assertion.

Next, if $\alpha+1 \rightarrow\left(\beta_{0}+1, \cdots\right)_{k}^{r+1}$, then, by Theorem 11 and the result just obtained, we conclude that

$$
\begin{aligned}
& 1+\alpha^{*} \rightarrow\left(1+\beta_{0}^{*}, 1+\beta_{1}^{*}, \cdots\right)_{k}^{1+r}, \\
& \alpha^{*} \rightarrow\left(\beta_{0}^{*}, \cdots\right)_{k}^{r} ; \quad \alpha \rightarrow\left(\beta_{0}, \cdots\right)_{k,}^{r} .
\end{aligned}
$$

Finally, let $1+a \rightarrow\left(1+b_{0}, 1+b_{1}, \cdots\right)_{k}^{1+r}$. Let $\alpha$ and $\beta_{\nu}$ be the initial ordinals belonging to $a$ and $b_{\nu}$ respectively. Then, by Theorems 14 and $13,1+\alpha \rightarrow\left(1+\beta_{0}, \cdots\right)_{k}^{1+r}$,

$$
\alpha \rightarrow\left(\beta_{0}, \cdots\right)_{k}^{r} ; \quad a \rightarrow\left(b_{0}, \cdots\right)_{k}^{r} .
$$

TheOREM 16. If $\alpha \rightarrow\left(\beta_{0}, \beta_{1}, \cdots\right)_{1+k}^{r} ; \beta_{0} \rightarrow\left(\gamma_{0}, \gamma_{1}, \cdots\right)_{l}^{r}$, then

$$
\alpha \rightarrow\left(\gamma_{0}, \gamma_{1}, \cdots, \beta_{1}, \beta_{2}, \cdots\right)_{l+k}^{r} .
$$

In this proposition the types $\alpha, \beta_{\mu}, \gamma_{\nu}$ may be replaced by cardinals.

In formulating the last theorem we use an obvious extension of the symbol (2).

Proof. We consider the case of types. Let $\bar{S}=\alpha$,

$$
[S]^{r}=\sum[\lambda<l] K_{0 \lambda}+\sum[0<\nu<1+k] K_{\nu} .
$$

Put $K_{0}=\sum[\lambda<l] K_{0 \lambda}$. Then, by hypothesis, there are $A \subset S ; \nu<1+k$ 
such that $\bar{A}=\beta_{v} ;[A]^{r} \subset K_{\nu}$. If $\nu>0$, then this is the desired conclusion. If $\nu=0$, then $\bar{A}=\beta_{0} ;[A]^{r} \subset \sum[\lambda<l] K_{0 \lambda}$ and so, by hypothesis, there are $B C A ; \lambda<l$ such that $\bar{B}=\gamma_{\lambda} ;[B]^{r} \subset K_{0 \lambda}$ which, again, is a conclusion of the desired kind. This proves the theorem.

It is clear that, instead of replacing in the relation $\alpha \rightarrow\left(\beta_{0}\right.$, $\left.\beta_{1}, \cdots\right)_{1+k}^{r}$ a single type $\beta_{0}$ by a well-ordered system of types $\gamma_{0}, \gamma_{1}, \cdots$, we could have replaced simultaneously every type $\beta_{\rho}$ by a system of types and in this way obtained a more general form of the transitive property of the partition relation than that given in Theorem 16.

THEOREM 17. If $\alpha \rightarrow\left(\beta_{0}, \beta_{1}, \cdots\right)_{k}^{r} ; \gamma_{\lambda}=\beta_{\rho_{\lambda}}(\lambda<l)$, where $\lambda \rightarrow \rho_{\lambda}$ is a one-one mapping of $[0, l]$ into $[0, k]$ such that $\beta_{\nu} \geqq r$ for $\nu \in[0, k]$ $-\left\{\rho_{\lambda}: \lambda<l\right\}$, then

$$
\alpha \rightarrow\left(\gamma_{0}, \gamma_{1}, \cdots\right)_{l}^{r} \text {. }
$$

In particular, the condition on the mapping $\lambda \rightarrow \rho_{\lambda}$ is satisfied whenever this mapping is on $[0, k]$.

The types $\alpha, \beta_{\nu}$ may be replaced by cardinals.

Proof. Let $N=\left\{\rho_{\lambda}: \lambda<l\right\}$, and let $\nu \rightarrow \sigma_{\nu}$ be the mapping of $N$ on $[0, l]$ which is inverse to the given mapping $\lambda \rightarrow \rho_{\lambda}$. Now let $\bar{S}=\alpha$; $[S]^{r}=\sum[\lambda<l] K_{\lambda}$. Then

$$
[S]^{r}=\sum[\nu \in N] K_{\sigma_{\nu}}+\sum[\nu \in[0, k]-N] 0 .
$$

By hypothesis, there are $A \subset S ; \nu<k$ such that $\bar{A}=\beta_{\nu}$ and either

$$
\nu \in N ; \quad[A]^{r} \subset K_{\sigma_{\nu}}
$$

or

$$
\nu \in N ; \quad[A] r=0 .
$$

In case (ii), $r \neq \bar{A}=\beta_{\nu}$ which contradicts the hypothesis. Hence (i) holds, $\bar{A}=\gamma_{\lambda} ;[A]^{r} \subset K_{\lambda}$, where $\lambda=\sigma_{\nu}<l$, and the result follows.

We note that the hypothesis relating to $\beta_{\nu} \geqq r$ cannot be omitted, as is seen from the pair of the obviously correct relations $4 \rightarrow(1,3,3)^{2}$; $4 \rightarrow(3,3)^{2}$.

CoROllary. If $\alpha \rightarrow(\beta)_{k}^{r} ;|k|=|l|$, then $\alpha \rightarrow(\beta)_{l}^{r}$.

This shows that, as far as $k$ is concerned, the truth of the relation $\alpha \rightarrow(\beta)_{k}^{r}$ depends only on $|k|$. We are therefore able to introduce the relation

$$
\alpha \rightarrow(\beta)_{d}^{r}
$$

which, by definition, holds if, and only if, 


$$
\alpha \rightarrow(\beta)_{k}^{r}
$$

for some, and hence for all, $k$ such that $|k|=d$. A similar remark applies to the relation

$$
a \rightarrow(b)_{d}^{r}
$$

TheOREM 18. Let $k<\omega_{0} ; \alpha \rightarrow\left(\beta_{0}, \beta_{1}, \cdots\right)_{k}^{r}$;

$$
\bar{S}=\alpha ; \quad[S]^{r}=K_{0}+\cdots+K_{k-1} .
$$

Then there are sets $M, N \subset[0, k]$ such that $|M|+|N|>k$,

$$
\beta_{\mu} \in\left[K_{\nu}\right] \quad \text { for } \mu \in M ; \nu \in N .
$$

In the special case $k=2$ we have either

$$
\beta_{0} \in\left[K_{0}\right]\left[K_{1}\right] \quad \text { or } \quad \text { (ii) } \beta_{1} \in\left[K_{0}\right]\left[K_{1}\right]
$$

or

$$
\beta_{0}, \beta_{1} \in\left[K_{0}\right] \quad \text { or } \quad \text { (iv) } \beta_{0}, \beta_{1} \in\left[K_{1}\right] .
$$

Proof. Let

$$
\begin{aligned}
& P_{\nu}=\left\{\mu: \mu<k ; \beta_{\mu} \in\left[K_{\nu}\right]\right\}, \\
& Q_{\nu}=[0, k]-P_{\nu}
\end{aligned}
$$$$
(\nu<k) .
$$

We have to find a set $N \subset[0, k]$ such that $\left|\Pi[\nu \in N] P_{\nu}\right|>k-|N|$ or, what is equivalent, $\left|\sum[\nu \in N] Q_{\nu}\right|<|N|$. If no such $N$ exists, i.e. if $\left|\sum[\nu \in N] Q_{\nu}\right| \geqq|N|$ for all $N \subset[0, k]$, then, by a theorem of P. Hall [8], it is possible to choose numbers $\rho_{\nu} \in Q_{\nu}$ such that $\rho_{\mu} \neq \rho_{\nu}$ $(\mu<\nu<k)$. Then $\beta_{\rho_{\nu}} \notin\left[K_{\nu}\right](\nu<k)$. On the other hand, by Theorem 17 and the hypothesis, $\alpha \rightarrow\left(\beta_{\rho_{0}}, \beta_{\rho_{1}}, \cdots\right)_{k}^{r}$. This is the required contradiction.

THEOREM 19. Let $\alpha \rightarrow(\beta, \gamma)^{2}$, and suppose that $m$ is the initial ordinal belonging to $|\alpha|$. Then at least one of the following four statements holds. ${ }^{4}$
(i) $\beta<\omega_{0}$
(ii) $\gamma<\omega_{0}$
(iii) $\beta, \gamma \leqq \alpha, m$
(iv) $\beta, \gamma \leqq \alpha, m^{*}$.

Proof. Let $S$ be a set ordered by means of the relation $x<y$ and also by means of the relation $x \ll y$, and let the corresponding order types of $S$ be $\bar{S}=\alpha ; \bar{S}_{<<}=m$. Then $[S]^{2}=K_{0}+^{\prime} K_{1}$ where $K_{0}=\{\{x$,

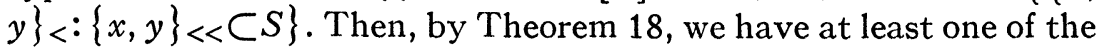
following four cases.

Case 1. $\beta \in\left[K_{0}\right]_{<}\left[K_{1}\right]_{<<}$. Then there are sets $A, B \subset S$ such that $\bar{A}_{<}=\bar{A}_{<<}=\beta ; \bar{B}_{<}=\bar{B}_{>>}=\beta$, and hence $\beta=\bar{A}_{<<} \leqq \bar{S}_{<<}=m$. Then $\beta$ is

4 (iii) means that $\beta \leqq \alpha ; \beta \leqq m ; \gamma \leqq \alpha ; \gamma \leqq m$. 
an ordinal. If $\beta \geqq \omega_{0}$, then the contradiction $\omega_{0}^{*} \leqq \beta^{*}=\bar{B}_{<<\leqq} \bar{S}_{<<}=m$ follows. Hence $\beta<\omega_{0}$.

Case 2. $\gamma \in\left[K_{0}\right]_{<}\left[K_{1}\right]_{<}$. Then, by symmetry, $\gamma<\omega_{0}$.

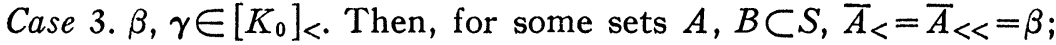
$\bar{B}_{<}=\bar{B}_{<<}=\gamma$, and $\beta, \gamma \leqq \alpha, m$.

Case 4. $\beta, \gamma \in\left[K_{1}\right]_{<}$. Then, similarly, $\bar{A}_{<}=\bar{A}_{>>}=\beta ; \bar{B}_{<}=\bar{B}_{>>}=\gamma ;$ $\beta, \gamma \leqq \alpha, m^{*}$. This proves the theorem.

Corollary. For every $\alpha$,

$$
(r-2)+\alpha \rightarrow\left(\omega_{0},(r-2)+\omega_{0}^{*}\right)^{r} \quad(r \geqq 2) .
$$

For none of the relations (i)-(iv) of Theorem 19 holds if $\beta=\omega_{0}$; $\gamma=\omega_{0}^{*}$. Hence $\alpha \rightarrow\left(\omega_{0}, \omega_{0}^{*}\right)^{2}$, and Theorem 15 yields (5).

The method employed in the proof of Theorem 19, i.e. the definition of a partition of $[S]^{2}$ from two given orders of $S$, seems to have been first used by Sierpiński [15]. In that note Sierpiński proves $\boldsymbol{\aleph}_{1} \rightarrow\left(\boldsymbol{\aleph}_{1}, \boldsymbol{\aleph}_{1}\right)^{2}$. Cf. Theorem 30 .

Theorem 20. (i) If $\beta_{0} \leqq \alpha ;\left|\beta_{0}\right|<r$, then $\alpha \rightarrow\left(\beta_{0}, \beta_{1}, \cdots\right)_{k}^{r}$ holds for any $k, \beta_{1}, \beta_{2}, \cdots$.

(ii) If $\beta_{\nu}=r$ for $\nu<k$, then the two relations

$$
\begin{aligned}
& \alpha \rightarrow\left(\beta_{0}, \beta_{1}, \cdots, \gamma_{0}, \gamma_{1}, \cdots\right)_{k+l}^{r}, \\
& \alpha \rightarrow\left(\gamma_{0}, \gamma_{1}, \cdots\right)_{l}^{r}
\end{aligned}
$$

are equivalent.

Proof of (i). If $\bar{S}=\alpha ;[S]^{r}=\sum[\nu<k] K_{\nu}$, then there is $A \subset S$ such that $\bar{A}=\beta_{0}$. Then $[A]^{r}=0 \subset K_{0}$.

Proof of (ii). By Theorem 12, (6) implies (7). Now suppose that (7) holds. Let $\bar{S}=\alpha$; $[S]^{r}=\sum[\nu<k+l] K_{\nu}$. If there is $\nu<k$ such that $K_{\nu} \neq 0$, then we can choose $A \in K_{\nu}$, and we shall have $A \subset S ; \bar{A}=\beta_{\nu}$; $[A]^{r} \subset K_{\nu}$. If, on the other hand, $K_{\nu}=0$ for all $\nu<k$, then $[S]^{r}$ $=\sum[\lambda<l] K_{k+\lambda}$, and there exist, by (7), $B \subset S ; \lambda<l$ such that $\bar{B}=\gamma_{\lambda} ;[B]^{r} C K_{k+\lambda}$. This proves (6).

Theorem 21. Let

$$
\alpha \rightarrow\left(\beta_{0}, \beta_{1}, \cdots\right)_{k}^{r} \text {. }
$$

Then either (i) there is $\nu_{0}<k$ such that $\beta_{\nu_{0}} \leqq \alpha$; $\left|\beta_{\nu_{0}}\right|<r$, or (ii) $\beta_{\nu} \leqq \alpha$ for all $\nu<k$.

REMARK. If (i) holds then (8) is true trivially. For, let $\bar{S}=\alpha$; $[S]^{r}=\sum[\nu<k] K_{\nu}$. Then we can choose $B C S$ such that $\bar{B}=\beta_{\nu_{0}}$. Then $[B]^{r}=0 \subset K_{\nu_{0}}$. We see, therefore, that the relation (8) need only be 
studied in the case in which $\beta_{\nu} \leqq \alpha$ for all $\nu<k$. In particular, if $\alpha$ is an an ordinal, then we may assume, if we wish, that every $\beta_{\nu}$ is an ordinal.

Proof. Suppose that (ii) is false. Then there is $\nu_{1}<k$ such that $\beta_{\nu_{1}} \neq \alpha$. Let $\bar{S}=\alpha$, and put $[S]^{r}=\sum^{\prime}[\nu<k] K_{\nu}$, where $K_{\nu_{1}}=[S]^{r}$. Then, by (8), there are $B C S ; \nu_{0}<k$ such that $\bar{B}=\beta_{\nu_{0}} ;[B]^{r} \subset K_{\nu_{0}}$. Then $\beta_{\nu_{0}}=\bar{B} \leqq \bar{S}=\alpha ; \nu_{0} \neq \nu_{1} ;[B]^{r}=0 ;\left|\beta_{\nu_{0}}\right|=|B|<r$. Hence (i) holds.

THEOREM 22. The following two tables give information about a number of cases in which the truth or otherwise of any of the relations

$$
\begin{aligned}
& \alpha \rightarrow\left(\beta_{0}, \beta_{1}, \cdots\right)_{k}^{r}, \\
& a \rightarrow\left(b_{0}, b_{1}, \cdots\right)_{k}^{r}
\end{aligned}
$$

can be decided trivially.

$$
k=0 \text { : }
$$

\begin{tabular}{|c|c|c|c|c|c|c|c|c|}
\hline & $\begin{array}{l}\beta_{\nu} \leqq \alpha \\
b_{\nu} \leqq a\end{array}$ & $\begin{array}{l}\beta_{\nu}=\alpha \\
b_{\nu}=a\end{array}$ & $\begin{array}{c}\beta_{\nu} \geqq \alpha ; \beta_{0} \ddagger \alpha \\
b_{\nu} \geqq a ; b_{0}>a\end{array}$ & $\begin{array}{l}\beta_{\nu} \$ \alpha \\
b_{\nu}>a\end{array}$ & $\begin{array}{l}\beta_{0} \geq \alpha \\
b_{0}<a\end{array}$ & $\begin{array}{l}\beta_{0} \leqq \alpha \\
b_{0} \leqq a\end{array}$ & $\begin{array}{l}\beta_{0} \underset{=}{ } \alpha \\
b_{0}>a\end{array}$ & $\begin{array}{l}\beta_{0} \geq r \\
b_{0}<r\end{array}$ \\
\hline$r=0$ & + & & & & & & - & \\
\hline $\begin{array}{l}0<r<|\alpha| \\
0<r<a\end{array}$ & & & & - & & \pm & & + \\
\hline $\begin{array}{l}r=|\alpha|>0 \\
r=a>0\end{array}$ & & + & - & & + & & & \\
\hline $\begin{array}{l}r>|\alpha| \\
r>a\end{array}$ & & & & - & & + & & \\
\hline
\end{tabular}

\begin{tabular}{|l|l|}
\hline$r \leqq|\alpha|$ & + \\
$r \leqq a$ & \\
\hline$r>|\alpha|$ & - \\
$r>a$ & \\
\hline
\end{tabular}

$$
k>0 \text { : }
$$


The proofs may be omitted. When a row or column is headed by two lines of conditions the first line refers to (9) and the second line to (10). Every condition involving the suffix $\nu$ is meant to hold for every $\nu<k$. An entry + means that both, (9) and (10) are true, and an entry - means that both, (9) and (10) are false. The one entry \pm marks the only case worth studying, i.e. the case in which (9) or (10) can be either true or false, and this for nontrivial reasons. In each row the entries are chosen in such a way that all possibilities are covered. In the column headings we may, of course, replace $\beta_{0}$ and $b_{0}$ by $\beta_{\nu_{0}}$ and $b_{\nu_{0}}$ respectively, for any choice of $\nu_{0}<k$. The case $k=0$ has, obviously, only curiosity value but is included for the sake of completeness.

\section{Denumerable order types.}

Theorem 23. If $n<\omega_{0} ; \alpha<\omega_{0} 2$, then

$$
\begin{aligned}
& \omega_{0} n \rightarrow(n, \alpha)^{2}, \\
& \omega_{0} n \rightarrow\left(n+1, \omega_{0}+1\right)^{2} .
\end{aligned}
$$

Proof. We may assume $n>0$.

(a) In order to prove (12), consider the set $S=\{(\nu, \lambda): \nu<n$; $\left.\lambda<\omega_{0}\right\}$, ordered alphabetically: $(\nu, \lambda)<\left(\nu_{1}, \lambda_{1}\right)$ if either (i) $\nu<\nu_{1}$ or (ii) $\nu=\nu_{1} ; \lambda<\lambda_{1}$. Then $[S]^{2}=K_{0}+^{\prime} K_{1}$, where $K_{1}$ is the set of all sets $\left\{(\nu, \lambda),\left(\nu, \lambda_{1}\right)\right\}<C S$. Then, clearly, $\bar{S}=\omega_{0} n ; n+1 \notin\left[K_{0}\right] ; \omega_{0}+1$ $\notin\left[K_{1}\right]$, and (12) follows.

(b) We now prove (11). Let the set $A=\sum[\nu<n] A_{\nu}$ be ordered, $\bar{A}_{\nu}=\omega_{0}$ for $\nu<n$, and $A_{\nu} \subset L\left(A_{\nu+1}\right)$ for $\nu+1<n$. Suppose that $[A]^{2}$ $=K_{0}+K_{1} ; n \notin\left[K_{0}\right] ; \alpha \notin\left[K_{1}\right]$. We want to deduce a contradiction.

By Theorem 1 , there is, for every $\nu<n$, a set $B_{\nu} \in\left[A_{\nu}\right]^{\aleph_{0}}$ such that $\left[B_{\nu}\right]^{2} \subset K_{\rho_{\nu}}$, for some $\rho_{\nu}<2$. Since $n \notin\left[K_{0}\right]$, we have $\rho_{\nu}=1$. Let $\lambda<\mu<n$. We define an operator $p_{\lambda \mu}$ as follows. There is at least one set $B \subset B_{\lambda}+B_{\mu}$ such that $\left|B B_{\lambda}\right|=\boldsymbol{\aleph}_{0} ;[B]^{2} \subset K_{1}$. For instance, $B_{\lambda}$ is such a set $B$. Since $\alpha \notin\left[K_{1}\right]$, we have, for every such $B, \omega_{0} \leqq \bar{B}<\alpha$ $<\omega_{0} 2$. Hence we can choose $B$ such that $\bar{B}$ is maximal. We fix such a $B$ by any suitable convention and put

$$
P_{\lambda \mu}\left(B_{0}, B_{1}, \cdots, B_{n-1}\right)=\left(C_{0}, C_{1}, \cdots, C_{n-1}\right),
$$

where $C_{\lambda}=B B_{\lambda} ; C_{\mu}=B_{\mu}-B$, and $C_{\nu}=B_{\nu}$ for $\nu \neq \lambda, \mu$. Then $\bar{C}_{\nu}=\omega_{0}$; $\left|C_{\lambda} L_{1}(x)\right|<\aleph_{0}$ for $\nu<n ; x \in C_{\mu}$. We now apply, in turn, all

$$
\left(\begin{array}{l}
n \\
2
\end{array}\right)
$$


operators $p_{\lambda \mu}$, corresponding to all choices of $\lambda, \mu$, to the system $\left(B_{0}, \cdots, B_{n-1}\right)$, applying each one of the operators, from the second onwards, to the system obtained by the preceding operator, and obtain, as end product, the system $\left(D_{0}, \cdots, D_{n-1}\right)$. Then $D_{\nu} \subset A_{\nu}$; $\bar{D}_{\nu}=\omega_{0}(\nu<n) ;\left|D_{\nu} L_{1}(x)\right|<\boldsymbol{\aleph}_{0}$ for $\nu<n ; x \in D_{\nu+1}+\cdots+D_{n-1}$. Hence it is possible to choose, in this order, elements $x_{n-1}, x_{n-2}, \cdots, x_{0}$ such that

$$
x_{\nu} \in D_{\nu} L_{0}\left(x_{\nu+1}, x_{\nu+2}, \cdots, x_{n-1}\right) \quad(\nu<n) .
$$

Then, putting $D=\left\{x_{\nu}: \nu<n\right\}$, we have $\bar{D}=n ;[D]^{2} \subset K_{0}$ and therefore $n \in\left[K_{0}\right]$ which is the required contradiction.

THEOREM 24. If $\alpha<\omega_{0} 4$, then

$$
\begin{aligned}
\alpha & \rightarrow\left(3, \omega_{0} 2\right)^{2}, \\
\omega_{0} 4 & \rightarrow\left(3, \omega_{0} 2\right)^{2} .
\end{aligned}
$$

This theorem is a special case of the following theorem.

Theorem 25. Let $2 \leqq m, n<\omega_{0}$, and denote by $l_{0}=l_{0}(m, n)$ the least finite number $l$ possessing the following property. ${ }^{5}$

Property $P_{m n}$. Whenever $\rho(\lambda, \mu)<2$ for $\{\lambda, \mu\}_{\neq} \subset[0, l]$, then there is either $\left\{\lambda_{0}, \cdots, \lambda_{m-1}\right\}_{\star} \subset[0, l]$ such that

$$
\rho\left(\lambda_{\alpha}, \lambda_{\beta}\right)=0 \quad \text { for } \alpha<\beta<m,
$$

or there is $\left\{\lambda_{0}, \cdots, \lambda_{n-1}\right\}_{\neq} \subset[0, l]$ such that

$$
\rho\left(\lambda_{\alpha}, \lambda_{\beta}\right)=1 \quad \text { for }\{\alpha, \beta\}_{\neq} \subset[0, n] .
$$

Then

$$
\begin{aligned}
\omega_{0} l_{0} & \rightarrow\left(m, \omega_{0} n\right)^{2}, & \\
\gamma & \rightarrow\left(m, \omega_{0} n\right)^{2} & \text { for } \gamma<\omega_{0} l_{0} .
\end{aligned}
$$

Moreover, if $l_{1} \rightarrow(m, m, n)^{2}$, then $l_{0} \leqq l_{1}$.

Deduction of Theorem 24 from Theorem 25. We have to prove that $l_{0}(3,2)=4$. (i) By considering the function $\rho$ defined by

$$
\rho(0,1)=\rho(1,2)=\rho(2,0)=0 ; \quad \rho(2,1)=\rho(1,0)=\rho(0,2)=1,
$$

we deduce that 3 does not possess the property $P_{32}$. (ii) Let us assume that 4 does not possess $P_{32}$. Then there is $\rho(\lambda, \mu)$ such that the condition stipulated for $P_{32}$ does not hold, with $l=4$. If

${ }^{5}$ The existence of such a number $l$ follows from Theorem 2. It will follow from Theorem 39 that we may take $l=\left(1+3^{2 m+n-5}\right) / 2$. 


$$
\{\alpha, \beta, \gamma\}_{*} \subset[0,4] ; \quad \rho(\alpha, \beta)=\rho(\alpha, \gamma)=0,
$$

then the assumption $\rho(\beta, \gamma)=0$ would lead to

$$
\rho(\alpha, \beta)=\rho(\beta, \gamma)=\rho(\alpha, \gamma)=0,
$$

i.e. to a contradiction. Hence $\rho(\beta, \gamma)=1$ and, by symmetry, $\rho(\gamma, \beta)$ $=1$. This, again, is a contradiction. This argument proves that

$$
\text { if }\{\alpha, \beta, \gamma\}_{\neq} \subset[0,4] ; \quad \rho(\alpha, \beta)=0, \quad \text { then } \rho(\alpha, \gamma)=1 .
$$

Since at least one of the numbers $\rho(0,1), \rho(1,0)$ is zero, there is a permutation $\alpha, \beta, \gamma, \delta$ of $0,1,2,3$ such that $\rho(\alpha, \beta)=0$. Then repeated application of (17) yields $\rho(\alpha, \gamma)=\rho(\alpha, \delta)=1 ; \rho(\gamma, \alpha)=0 ; \rho(\gamma, \delta)=1$; $\rho(\delta, \alpha)=\rho(\delta, \gamma)=0$, which contradicts (17). This proves $l_{0}(3,2) \leqq 4$ and, in conjunction with (i), $l_{0}(3,2)=4$.

ProOF OF THEOREM 25. 1 . We begin by proving the last clause. Let

$$
l_{1} \rightarrow(m, m, n)^{2} \text {. }
$$

Suppose that $\rho(\lambda, \mu)<2$ for $\{\lambda, \mu\}_{\neq} \subset\left[0, l_{1}\right]$. Then

$$
[S]^{2}=K_{0}+K_{1}+K_{2}
$$

where $S=\left[0, l_{1}\right]$, and $K_{\nu}$ is the set of all $\{\lambda, \mu\}_{<} \subset\left[0, l_{1}\right]$ such that

$$
\begin{array}{ll}
\rho(\lambda, \mu)=0 & (\nu=0), \\
\rho(\lambda, \mu)>\rho(\mu, \lambda) & (\nu=1), \\
\rho(\lambda, \mu)=\rho(\mu, \lambda)=1 & (\nu=2) .
\end{array}
$$

By (18), there is $S_{1}=\left\{\lambda_{0}, \cdots, \lambda_{k-1}\right\}<C S$ such that one of the following three statements holds.

$$
\begin{aligned}
& k=m ; \quad\left[S_{1}\right]^{2} \subset K_{0}, \\
& k=m ; \quad\left[S_{1}\right]^{2} \subset K_{1}, \\
& k=n ; \quad\left[S_{1}\right]^{2} \subset K_{2} \text {. }
\end{aligned}
$$

(19) implies that $\rho\left(\lambda_{\alpha}, \lambda_{\beta}\right)=0$ for $\alpha<\beta<m$;

(20) implies that $\rho\left(\lambda_{m-1-\alpha}, \lambda_{m-1-\beta}\right)=0$ for $\alpha<\beta<m$;

(21) implies that $\rho\left(\lambda_{\alpha}, \lambda_{\beta}\right)=1$ for $\{\alpha, \beta\}_{\neq} \subset[0, n]$.

This shows that $l_{0}(m, n) \leqq l_{1}$.

2. We now prove (15). Let $l=l_{0}(m, n) ; A=\left[0, \omega_{0} l\right] ; N=\left[0, \omega_{0}\right]$;

$$
[A]^{2}=K_{0}+{ }^{\prime} K_{1}
$$

(partition $\Delta$ ).

We use the notation of the partition calculus given in detail in $[4$, p. 419] which can be summarized as follows. If $\Delta$ is an equivalence 
relation on a set $M$ or a partition of $M$ into disjoint classes then $|\Delta|$ denotes the cardinal of the set of nonempty classes, and the relation

$$
x \equiv y(\cdot \Delta)
$$

expresses the fact that $x$ and $y$ belong to $M$ and lie in the same class of $\Delta$. If, for $\rho \in R, \Delta_{\rho}$ is a partition of $M$, and if $t \rightarrow f_{\rho}(t)$ is a mapping of a set $T$ into $M$, then the formula

$$
\Delta^{\prime}(t)=\prod[\rho \in R] \Delta_{\rho}\left(f_{\rho}(t)\right)
$$

defines that partition $\Delta^{\prime}$ of $T$ for which

$$
s \equiv t\left(\cdot \Delta^{\prime}\right)
$$

if, and only if,

$$
f_{\rho}(s) \equiv f_{\rho}(t)(\cdot \Delta) \quad \text { for } \rho \in R .
$$

We continue the proof of (15) by putting

$$
\Delta^{\prime}(\{\sigma, \tau\})=\prod[\lambda, \mu<l] \Delta\left(\left\{\omega_{0} \lambda+\sigma, \omega_{0} \mu+\tau\right\}\right) \quad\left(\sigma<\tau<\omega_{0}\right) .
$$

By Theorem 1 there is $N^{\prime} \in[N]^{\aleph_{0}}$ such that $\left|\Delta^{\prime}\right|=1$ in $\left[N^{\prime}\right]^{2}$. Then, by definition of $\Delta^{\prime}$, there is $\rho(\lambda, \mu)<2$ such that

$$
\left\{\omega_{0} \lambda+\sigma, \omega_{0} \mu+\tau\right\} \in K_{\rho(\lambda, \mu)} \quad \text { for } \lambda, \mu<l ;\{\sigma, \tau\}_{<} \subset N^{\prime} .
$$

By definition of $l$ this implies that there is a set $\left\{\lambda_{0}, \cdots, \lambda_{k-1}\right\}_{\neq}$ $C[0, l]$ such that either

$$
k=m ; \quad \rho\left(\lambda_{\alpha}, \lambda_{\beta}\right)=0 \quad \text { for } \alpha<\beta<m
$$

or

$$
k=n ; \quad \rho\left(\lambda_{\alpha}, \lambda_{\beta}\right)=1 \quad \text { for }\{\alpha, \beta\}_{\neq} \subset[0, n] .
$$

If (22) holds, then we put

$$
A^{\prime}=\left\{\omega_{0} \lambda_{\alpha}+\sigma_{\alpha}: \alpha<m\right\},
$$

where $\sigma_{\alpha}$ is chosen such that $\left\{\sigma_{0}, \sigma_{1}, \cdots, \sigma_{m-1}\right\}<C N^{\prime}$. Then $\left[A^{\prime}\right]^{2} \subset K_{0}$, so that the desired conclusion $m \in\left[K_{0}\right]$ is reached.

If we now assume that $m \notin\left[K_{0}\right]$, then $\rho(\lambda, \lambda)=1$ for $\lambda<l$, and, furthermore, (23) holds. Then we put $N^{\prime}=\left\{\sigma_{0}, \sigma_{1}, \cdots\right\}<\bar{A}^{\prime \prime}$ $=\left\{\omega_{0} \lambda_{\alpha}+\sigma_{\alpha+t n}: \alpha<n ; t<\omega_{0}\right\}$. We find that $\left[A^{\prime \prime}\right]^{2}<K_{1} ; \omega_{0} n=A^{\prime \prime}$ $\in\left[K_{1}\right]$. This proves (15).

3. Finally, let $\gamma<\omega_{0} l_{0}$. Then there is $l<l_{0}$ such that $\omega_{0} l$ $\leqq \gamma<\omega_{0}(l+1)$. Then, by definition of $l_{0}$, there is $\rho(\lambda, \mu)<2$ for $\{\lambda, \mu\}_{\circledast} \subset[0, l]$ such that, whenever $\left\{\lambda_{0}, \cdots, \lambda_{m-1}\right\}_{\star} \subset[0, l]$, then

$$
\rho\left(\lambda_{\alpha}, \lambda_{\beta}\right) \neq 0
$$


for some $\{\alpha, \beta\}_{<} \subset[0, m]$, and, whenever $\left\{\lambda_{0}, \cdots, \lambda_{n-1}\right\}_{*} \subset[0, l]$, then

$$
\rho\left(\lambda_{\alpha}, \lambda_{\beta}\right) \neq 1
$$

for some $\{\alpha, \beta\}_{*} \subset[0, n]$. Then, if $A=[0, \gamma]$, we have $[A]^{2}=K_{0}+^{\prime} K_{1}$, where $K_{0}$ is the set of all $\left\{\omega_{0} \lambda+\sigma, \omega_{0} \mu+\tau\right\}$ such that $\{\lambda, \mu\}_{\star} \subset[0, l]$; $\sigma<\tau<\omega_{0} ; \rho(\lambda, \mu)=0$. If, now, $A^{\prime} \in[A]^{m} ;\left[A^{\prime}\right]^{2} \subset K_{0}$, then

$$
\begin{array}{rlrl}
A^{\prime} & =\left\{\omega_{0} \lambda_{\alpha}+\sigma_{\alpha}: \alpha<m\right\} ; & \sigma_{0}<\cdots<\sigma_{m-1}<\omega_{0} ; \\
\left\{\lambda_{0}, \cdots, \lambda_{m-1}\right\}_{*} & \subset[0, l] ; & & \text { for } \alpha<\beta<m, \\
\rho\left(\lambda_{\alpha}, \lambda_{\beta}\right) & =0 & & \text { for }
\end{array}
$$

which contradicts (24). If, on the other hand,

$$
A^{\prime \prime} \subset A ; \quad \bar{A}^{\prime \prime}=\omega_{0} n ; \quad\left[A^{\prime \prime}\right]^{2} \subset K_{1},
$$

then there is $\left\{\lambda_{0}, \cdots, \lambda_{n-1}\right\}<C[0, l]$ such that $\bar{B}_{\alpha}=\omega_{0}$ for $\alpha<n$, where $B_{\alpha}=A^{\prime \prime}\left[\omega_{0} \lambda_{\alpha}, \omega_{0}\left(\lambda_{\alpha}+1\right)\right] \quad(\alpha<n)$. Then $\rho\left(\lambda_{\alpha}, \lambda_{\beta}\right)=1$ for $\{\alpha, \beta\}_{\star} \subset[0, n]$, which contradicts (25). Hence neither $A^{\prime}$ nor $A^{\prime \prime}$ exist, with the properties stated, so that (16) follows. This completes the proof of Theorems 24 and 25.

6. The linear continuum. Our object is to investigate relations of the form

$$
\lambda \rightarrow\left(\alpha_{0}, \alpha_{1}, \cdots\right)_{k}^{r}
$$

and their negatives. It turns out ${ }^{6}$ that every positive relation we were able to prove holds not only for the particular type $\lambda$ of the set of all real numbers but for every type $\phi$ such that

$$
|\phi|>\aleph_{0} ; \quad \omega_{1}, \omega_{1}^{*} \$ \phi .
$$

This fact seems to suggest that, given any type $\phi$ satisfying (26), there always exists $\lambda_{1}$ such that

$$
\lambda_{1} \leqq \lambda, \phi ; \quad\left|\lambda_{1}\right|>\boldsymbol{\aleph}_{0},
$$

i.e., that every nondenumerable type which does not "contain" $\omega_{1}$ or $\omega_{1}^{*}$ contains a nondenumerable type which is embeddable in the real continuum. This conjecture has, as far as the authors are aware, neither been proved nor disproved. ${ }^{7}$

Throughout this section $S$ denotes the set of all real numbers $x$ such that $0<x<1$, ordered by magnitude. The letters $x, y, z$ denote elements of $S$, and $\lambda=\bar{S}$.

6 Cf. Theorems 31, 32.

${ }^{7}$ Since this paper was submitted E. Specker has disproved this conjecture. 
THEOREM 26.

$$
\begin{aligned}
& \lambda+\left(\omega_{1}\right)_{k}^{r} \\
& \lambda+(r+1)_{\omega_{0}}^{r}
\end{aligned}
$$

$$
\text { for } r \geqq 0 ; k>0 .
$$

Proof. (i) is trivial, in view of $\omega_{1} \leq \lambda$. In order to prove (ii) it suffices, by Theorem 15, to consider the case $r=2$. Let $\left\{x_{\nu}: \nu<\omega_{0}\right\}$ be the set of all rational numbers in $S$, and denote, for $n<\omega_{0}$, by $K_{n}$ the set of all $\{x, y\}<$ such that the least $\nu$ satisfying $x<x_{\nu}<y$ is $\nu=n$. Then $[S]^{2}=\sum\left[\nu<\omega_{0}\right] K_{\nu}$. Also, if $\left[\{x, y, z\}_{<}\right]^{2} \subset K_{n}$, then the contradiction $x<x_{n}<y<x_{n}<z$ follows. Hence $3 \notin\left[K_{n}\right]$, and Theorem 26 is proved.

THEOREM 27. $\lambda \rightarrow\left(\omega_{0}, \omega_{0}+2\right)^{r}$ for $r \geqq 3$.

Proof. By Theorem 15, we need only consider the case $r=3$. We have $[S]^{3}=K_{0}+K_{1}$, where $K_{0}=\left\{\{x, y, z\}_{<}: y-x<z-y\right\}$.

Assumption 1. Let $\left[\left\{x_{0}, x_{1}, \cdots\right\}<\right]^{3} \subset K_{0}$. Then $\lim x_{\nu}=u$ as $\nu \rightarrow \infty$, and we have, for $0<m<\omega_{0}$,

$$
\left\{x_{0}, x_{m}, x_{m+1}\right\} \in K_{0} ; \quad x_{m}-x_{0}<x_{m+1}-x_{m} .
$$

If $m \rightarrow \infty$, then the contradiction $u-x_{0} \leqq u-u$ follows.

Assumption 2. Let $A \subset S ; \bar{A}=\omega_{0}+2 ;[A]^{3} \subset K_{1}$. Then $A$ $=B+\{y, z\}_{<; B} B=\left\{x_{0}, x_{1}, \cdots\right\}_{<} \subset L(y) ; \lim x_{\nu}=u$ as $\nu \rightarrow \infty$, and we have, for $m<\omega_{0},\left\{x_{m}, x_{m+1}, z\right\} \in K_{1} ; x_{m+1}-x_{m} \geqq z-x_{m+1}$. If $m \rightarrow \infty$, then the contradiction $u-u \geqq z-u$ follows. This proves Theorem 27 .

THEOREM 28. $\lambda \rightarrow\left(r+1, \omega_{0}+2\right)^{r}$ for $r \geqq 4$.

Proof. It suffices to consider the case $r=4$. We have $[S]^{4}$ $=K_{0}+^{\prime} K_{1}$, where $K_{0}=\left\{\left\{x_{0}, x_{1}, x_{2}, x_{3}\right\}<: x_{2}-x_{1}<x_{3}-x_{2}, x_{1}-x_{0}\right\}$.

Assumption 1. Let $\left[\left\{x_{0}, x_{1}, x_{2}, x_{3}, x_{4}\right\}_{<}\right]^{4} \subset K_{0}$. Then $\left\{x_{0}, x_{1}, x_{2}, x_{3}\right\}$ $\in K_{0}$, and hence $x_{2}-x_{1}<x_{3}-x_{2}$. Also, $\left\{x_{1}, x_{2}, x_{3}, x_{4}\right\} \in K_{0}$, and hence $x_{3}-x_{2}<x_{2}-x_{1}$. This is a contradiction.

Assumptron 2. Let $A \subset S ; \bar{A}=\omega_{0}+2 ;[A]^{4} \subset K_{1}$. We define $B, y$, $z, x_{\nu}, u$ as in the proof of Theorem 27. Then there is $m_{0}<\omega_{0}$ such that, for $m_{0} \leqq m<\omega_{0}, u-x_{m}<x_{m}-x_{0}$. Then, for $m_{0} \leqq m<\omega_{0},\left\{x_{0}, x_{m}, x_{m+1}, z\right\}$ $\in K_{1} ; x_{m+1}-x_{m}<u-x_{m}<x_{m}-x_{0} ; x_{m+1}-x_{m} \geqq z-x_{m+1}$, and if $m \rightarrow \infty$, then the contradiction $u-u \geqq z-u$ follows. This proves Theorem 28 .

The next two theorems are extensions of results due to Sierpinski.

Theorem 29. If $2 \leqq|k| \leqq|\lambda| ;\left|\alpha_{\nu}\right| \geqq|\lambda|(\nu<k)$, then

$$
\lambda \rightarrow\left(\alpha_{0}, \alpha_{1}, \cdots\right)_{k}^{1} \text {. }
$$

Sierpinski proved that $\lambda \leftrightarrow(\alpha, \alpha)^{1}$ if $\alpha \leqq \lambda ;|\alpha|=|\lambda|$ (Theorem 9). 
Proof.

Case 1. There is $\mu<k$ such that $\alpha_{\mu} \ddagger \lambda$. We consider the partition $S=\sum^{\prime}[\nu<k] K_{\nu}$, where $K_{\mu}=S$. We have $\alpha_{\mu} \ddagger \lambda=\bar{K}_{\mu}$ and, for $\nu \neq \mu$, $\alpha_{\nu} \$ 0=\bar{K}_{\nu}$. Hence $\alpha_{\nu} \$ \bar{K}_{\nu}(\nu<k)$.

Case 2. $\alpha_{\nu} \leqq \lambda(\nu<k)$. We choose a fixed set $A_{0} \subset S$ such that $\bar{A}_{0}=\alpha_{0}$. Generally, the letter $A$ denotes sets such that $A \subset S ; \bar{A}=\alpha_{0}$. Corresponding to every $A$, there is a real function $f_{A}(x)$, defined and strictly increasing for $x \in A_{0}$, such that $x \rightarrow f_{A}(x)$ is a mapping of $A_{0}$ on $A$. We extend the definition of $f_{A}$ by putting $f_{A}(x)=0$ for $x \in L\left(A_{0}\right)$ and

$$
f_{A}(x)=\sup \left[y \leqq x ; y \in A_{0}\right] f_{A}(y) \quad \text { for } x \notin L\left(A_{0}\right) .
$$

Then $f_{A}(x)$ is nondecreasing in $S$. The set $A$ is uniquely determined by the function $f_{A}$ and the set $A_{0}$. Let $D(A)$ be the set of those $x_{0}$ for which $f_{A}(x)$ is discontinuous at $x=x_{0}$. Then $|D(A)| \leqq \aleph_{0}$. The function $f_{A}$ is uniquely determined by (i) the set $D(A)$ and (ii) the values of $f_{A}(x)$ for $x \in D(A)$ and (iii) the values of $f_{A}(x)$ for all rational $x$. Therefore

$$
\left|\sum\{A\}\right|=\left|\sum\left\{f_{A}\right\}\right| \leqq|\lambda| 3 \aleph_{0}=|\lambda| \leqq\left|\sum\{A\}\right|,
$$

and $\left|\sum\{A\}\right|=|\lambda|=\boldsymbol{N}_{n}$, say. Now we can write $\sum\{A\}=\left\{A_{0_{\rho}}\right.$ : $\left.\rho<\omega_{n}\right\}$. By symmetry, we have, for every $\nu<k$, a set $\left\{A_{\nu \rho}: \rho<\omega_{n}\right\}$ whose elements are all subsets of $S$ of type $\alpha_{\nu}$.

The set $N=\left\{(\nu, \rho): \nu<k ; \rho<\omega_{n}\right\}$ satisfies $|N|=|k| \boldsymbol{\aleph}_{n}=\boldsymbol{\aleph}_{n}$. We order $N$ in such a way that $\bar{N}=\omega_{n}$. Then we can find, inductively, $x_{\nu \rho}$ such that, for $(\nu, \rho) \in N$,

$$
x_{\nu \rho} \in A_{\nu \rho}-\left\{x_{\mu \sigma}:(\mu, \sigma)<(\nu, \rho)\right\} .
$$

For, $|\{(\mu, \sigma):(\mu, \sigma)<(\nu, \rho)\}|<|N| \leqq\left|A_{\nu \rho}\right|$. We have $x_{\nu \rho} \neq x_{\mu \sigma}$ for $(\nu, \rho) \neq(\mu, \sigma)$. Now, $S=\sum[\nu<k] K_{\nu}$, where $K_{\nu}=S-\left\{x_{\nu \rho}: \rho<\omega_{n}\right\}$. For, if $x \in S$, then, since $k \geqq 2$, there is $\nu<k$ such that $x \in K_{\nu}$. If, now, $\alpha_{\nu} \leqq \bar{K}_{\nu}$ for some $\nu<k$, then there is $\rho$ such that $x_{\nu \rho} \in A_{\nu \rho} \subset K_{\nu}$, which is the required contradiction. This proves Theorem 29.

THEOREM 30. $|\lambda| \rightarrow\left(\boldsymbol{\aleph}_{1}, \boldsymbol{\aleph}_{1}\right)^{r}$ for $r \geqq 2$.

Proof. The substance of this theorem is due to Sierpiński [15]. By Theorem 15 we need only consider the case $r=2$. Let $x<y$ be, as throughout this section, the order of $S$ by magnitude, and let $x \ll y$ be an order of $S$ such that $\bar{S}_{<<}=\omega_{n}$, where $|\lambda|=\boldsymbol{\aleph}_{n}$. Then $[S]^{2}$ $=K_{0}+{ }^{\prime} K_{1}$, where $K_{0}=\left\{\{x, y\}_{<}: x \ll y\right\}$. Now let $A \subset S ;[A]^{2} \subset K_{\nu}$.

If $\nu=0$, then $\bar{A}_{<} \leqq \bar{S}_{<}=\lambda ; \bar{A}_{<}=\bar{A}_{<<} \leqq \bar{S}_{<<}=\omega_{n}$, and hence $\bar{A}_{<}<\omega_{1}$;

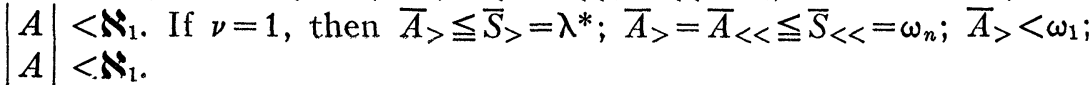


This proves Theorem 30 . We note that this theorem is, in fact, an easy corollary of [5, Example $4 \mathrm{~A}]$.

7. The general case. We shall consider relations involving certain types of cardinal $\boldsymbol{\aleph}_{1}$ as well as relations between types of any cardinal. We begin by proving a lemma. We establish this lemma in a form which is more general than will later be required, but in this form it seems to possess some interest of its own. We recall that $a^{\prime}$ denotes the cofinality cardinal belonging to $a$ which was defined in $\$ 2$.

LeммA 1. Let $S$ be an ordered set, and $|S|^{\prime}=\aleph_{n} ; \omega_{n}, \omega_{n}^{*} \bar{S}$. Then, corresponding to every rational number $t$, there is $S_{t} \subset S$ such that $\left|S_{t}\right|=|S| ; S_{t} \subset L\left(S_{u}\right)$ for $t<u$.

Sierpiński, in a letter to one of us, had already noted the weaker result that, if $|S|=\aleph_{1} ; \omega_{1}, \omega_{1}^{*} \$ \bar{S}$, then $\eta \leqq \bar{S}$.

Proof. Case 1. There is $A \subset S$ such that

$$
|A L(x)|<|A|=|S| \quad(x \in A) \text {. }
$$

Then we define $x_{\nu}$ for $\nu<\omega_{n}$ inductively as follows. Let $\nu_{0}<\omega_{n}$; $x_{\nu} \in A\left(\nu<\nu_{0}\right)$. Then, by definition of $n$,

$$
\left|\sum\left[\nu<\nu_{0}\right]\left(A L\left(x_{\nu}\right)+\left\{x_{\nu}\right\}\right)\right|<|A| \text {, }
$$

and hence there is $x_{\nu_{0}} \in A-\sum\left[\nu<\nu_{0}\right]\left(L\left(x_{0}\right)+\left\{x_{\nu}\right\}\right)$. Then $x_{\mu}<x_{\nu}$ $\left(\mu<\nu<\omega_{n}\right)$ and so $\omega_{n} \leqq \bar{S}$, which is false.

Case 2. There is $A \subset S$ such that

$$
|A R(x)|<|A|=|S| \quad(x \in A) .
$$

Then, by symmetry, the contradiction $\omega_{n}{ }^{*} \leqq \bar{S}$ follows.

Case 3. There is $A \subset S$ such that

$$
\min (|A L(x)|,|A R(x)|)<|A|=|S| \quad(x \in A) .
$$

Then we put

$$
\begin{aligned}
& A_{0}=\{x: x \in A ;|A L(x)|<|A|\}, \\
& A_{1}=\{x: x \in A ;|A R(x)|<|A|\} .
\end{aligned}
$$

Then $A=A_{0}+A_{1}$.

Case 3.1. $\left|A_{0}\right|=|S|$. Then $\left|A_{0} L(x)\right| \leqq|A L(x)|<|S|=\left|A_{0}\right|$ $\left(x \in A_{0}\right)$, and hence, by Case 1 , we find a contradiction.

Case 3.2. $\left|A_{0}\right| \neq|S|$. Then $\left|A_{1}\right|=|S|$ and, by symmetry, a contradiction follows.

We have so far proved that, if $A \subset S ;|A|=|S|$, there is $z \in A$ such that $|A L(z)|=|A R(z)|=|S|$. Then $A=A^{\prime}+A^{\prime \prime}$, where $A^{\prime}=A L(z)$; 
$\left|A^{\prime}\right|=\left|A^{\prime \prime}\right|=|S| ; A^{\prime} \subset L\left(A^{\prime \prime}\right)$. By applying this result to $A^{\prime}$ we find a partition $A=A(0)+A(1)+A(2)$ such that

$$
|A(\nu)|=|S|(\nu<3) ; A(\nu) \subset L(A(\nu+1)) \quad(\nu<2) .
$$

Repeated application leads to sets

$$
A\left(\lambda_{0}, \lambda_{1}, \cdots, \lambda_{k-1}\right) \quad\left(k<\omega_{0} ; \lambda_{\nu}<3\right)
$$

such that

$$
\begin{aligned}
\left|A\left(\lambda_{0}, \cdots, \lambda_{k-1}\right)\right| & =|S| \\
A\left(\lambda_{0}, \cdots, \lambda_{k-1}\right) & =\sum[\nu<3] A\left(\lambda_{0}, \cdots, \lambda_{k-1}, \nu\right) ; \\
A\left(\lambda_{0}, \cdots, \lambda_{k-1}, \nu\right) & \subset L\left(A\left(\lambda_{0}, \cdots, \lambda_{k-1}, \nu+1\right)\right) \quad(\nu<2) .
\end{aligned}
$$

Let $N$ be the set of all systems $\left(\lambda_{0}, \cdots, \lambda_{k}\right)$ such that $k<\omega_{0}$; $\lambda_{\nu} \in\{0,2\}(\nu<k) ; \lambda_{k}=1$, ordered alphabetically. More accurately, if $p=\left(\lambda_{0}, \cdots, \lambda_{k}\right)$ and $q=\left(\mu_{0}, \cdots, \mu_{l}\right)$ are elements of $N$, then we put $p<q$ if $\sum[\nu<k] \lambda_{\nu} 3^{-\nu}<\sum[\nu<l] \mu_{\nu} 3^{-\nu}$. Then we have $A\left(\lambda_{0}, \cdots, \lambda_{k}\right)$ $C L\left(A\left(\mu_{0}, \cdots, \mu_{l}\right)\right)$, if $\left(\lambda_{0}, \cdots\right)<\left(\mu_{0}, \cdots\right)$. It now suffices to show that $N$ is dense in itself. In fact, if $\left\{\left(\lambda_{0}, \cdots, \lambda_{k}\right),\left(\mu_{0}, \cdots, \mu_{l}\right)\right\}<\subset$, then

$$
\left(\lambda_{0}, \cdots, \lambda_{k}\right)<\left(\mu_{0}, \cdots, \mu_{l-1}, 0,2,2, \cdots, 2,1\right)<\left(\mu_{0}, \cdots, \mu_{l}\right),
$$

provided only that the inner bracket contains a sufficiently large number of two's. Lemma 1 is proved.

THEOREM 31. Suppose that $\phi$ is a type such that

$$
|\phi|>\aleph_{0} ; \quad \omega_{1}, \omega_{1}^{*} \$ \phi .
$$

Let $\alpha<\omega_{0} 2 ; \beta<\omega_{0}^{2} ; \gamma<\omega_{1}$. Then

$$
\begin{aligned}
& \phi \rightarrow(\alpha, \alpha, \alpha)^{2}, \\
& \phi \rightarrow(\alpha, \beta)^{2}, \\
& \phi \rightarrow\left(\omega_{0}, \gamma\right)^{2}, \\
& \phi \rightarrow(4, \alpha)^{3} .
\end{aligned}
$$

TheOREM 32. Let $\phi, \alpha, \gamma$ be as in Theorem 31. Let $S$ be an ordered set, $\bar{S}=\phi$, and $[S]^{2}=K_{0}+K_{1}$. Then

(a) there is $V \subset S$ such that either

$$
\text { (i) } \bar{V}=\alpha ; \quad[V]^{2} \subset K_{0} \text {, }
$$

or

(ii) $\bar{V}=\gamma ; \quad[V]^{2} \subset K_{1}$, 
or

$$
\text { (iii) } \bar{V}=\omega_{0} \gamma^{*} ; \quad[V]^{2} \subset K_{1} \text {, }
$$

and

(b) there is $W \subset S$ such that either

$$
\text { (i) } \bar{W}=\omega_{0}+\stackrel{*}{\omega_{0}} ; \quad[W]^{2} \subset K_{0}
$$

or

$$
\text { (ii) } \bar{W}=\gamma ; \quad[W]^{2} \subset K_{1} \text {, }
$$

or

$$
\text { (iii) } \bar{W}=\gamma^{*} ; \quad[W]^{2} \subset K_{1} \text {. }
$$

In proving Theorems 31 and 32 we may assume that $|\phi|=\boldsymbol{\aleph}_{1}$. There is $m$ such that

$$
4 \leqq m<\omega_{0} ; \quad \alpha \leqq \omega_{0}+m ; \quad \beta \leqq \omega_{0} m .
$$

Let $\bar{S}=\phi$. The letters $A, B, P, Q$ denote subsets of $S$, and we shall always suppose, in the proofs of the last two theorems, that

$$
|A|=|B|=\boldsymbol{N}_{1} ; \quad \bar{P}=\bar{Q}=\omega_{0} .
$$

Proof of Theorem 31, (29). Let $[S]^{2}=K_{0}+K_{1}$, and

$$
\omega_{0} \notin\left[K_{0}\right] \text {. }
$$

We want to deduce that

$$
\gamma \in\left[K_{1}\right]
$$

There is $B$ such that

$$
\left|B R_{0}(x)\right| \leqq \boldsymbol{N}_{0}(x \in B)
$$

For otherwise there would be elements $x_{\nu}$ such that

$$
\begin{array}{llrl}
x_{0} \in S ; & \left|R_{0}\left(x_{0}\right)\right| & =\aleph_{1}, \\
x_{1} \in R_{0}\left(x_{0}\right) ; & \left|R_{0}\left(x_{0}, x_{1}\right)\right| & =\aleph_{1},
\end{array}
$$

generally, $x_{\nu} \in R_{0}\left(x_{0}, \cdots, x_{\nu-1}\right)$,

$$
\left|R_{0}\left(x_{0}, \cdots, x_{\nu}\right)\right|=\aleph_{1} \quad\left(\nu<\omega_{0}\right) .
$$

Then $\left[\left\{x_{0}, x_{1}, \cdots\right\}\right]_{<}^{2} \subset K_{0}$, and hence $\omega_{0} \in\left[K_{0}\right]$ which contradicts (31).

By hypothesis, $\left.\omega_{1}, \omega_{1}^{*}\right\rfloor \bar{B}$. Hence, by Lemma 1 , 


\section{$\sum[t$ rational $] B(t) \subset B$}

for some sets $B(t)$ such that $B(t) \subset L(B(u))(t<u)$.

There is a set $T$ of rational numbers which, if ordered by magnitude, is of type $\gamma$. Let $T=\left\{t_{\mu}: \mu<\gamma\right\}$ where $t_{\mu}<t_{\nu}$ for $\mu<\nu<\gamma$. We define inductively elements $x_{\nu}(\nu<\gamma)$ as follows. Let $\nu_{0}<\gamma$, and suppose that $x_{\nu} \in B\left(\nu<\nu_{0}\right)$. Then, by (33),

$$
\left|\sum\left[\nu<\nu_{0}\right] B R_{0}\left(x_{\nu}\right)\right| \leqq \aleph_{0}<\left|B\left(t_{\nu_{0}}\right)\right|,
$$

and therefore we can choose $x_{\nu_{0}} \in B\left(t_{\nu_{0}}\right)-\sum\left[\nu<\nu_{0}\right] R_{0}\left(x_{\nu}\right)$. Put $X=\left\{x_{\nu}: \nu<\gamma\right\}$. Then $\bar{X}=\gamma ;[X]^{2} \subset K_{1}$. Hence (32) holds, and (29) follows.

Proof of Theorem 32 (a). Let the hypotheses be satisfied but suppose that (a) is false, i.e. that

$$
\alpha \notin\left[K_{0}\right] ; \quad \gamma \in\left[K_{1}\right] ; \quad \omega_{0} \gamma^{*} \notin\left[K_{1}\right] .
$$

Assumption. If $A \subset S$, then there is $x_{0} \in A$ such that

$$
\left|A L_{0}\left(x_{0}\right)\right|>\boldsymbol{\aleph}_{0} .
$$

Then there are $x_{\nu}, A_{\nu}(\nu \leqq m)$ such that

$$
x_{0} \in A_{0}=S ; \quad A_{0} L_{0}\left(x_{0}\right)=A_{1} ; \quad x_{1} \in A_{1} ; \quad A_{1} L_{0}\left(x_{1}\right)=A_{2}
$$

and so on, up to

$$
A_{m}=A_{m-1} L_{0}\left(x_{m-1}\right)=A_{0} L_{0}\left(x_{0}, x_{1}, \cdots, x_{m-1}\right) .
$$

Then, by (29), $\bar{A}_{m} \rightarrow\left(\omega_{0}, \gamma\right)^{2} ; \gamma \notin F_{1}\left(A_{m}\right)$, and hence $\omega_{0} \in F_{0}\left(A_{m}\right)$. There is $P \subset A_{m}$ such that $[P]^{2} \subset K_{0}$. Then $\left[P+\left\{x_{0}, \cdots, x_{m-1}\right\}\right]^{2}$ $\subset K_{0}$ which contradicts (34). Hence our assumption is false, and there is $A$ such that

$$
\left|A L_{0}(x)\right| \leqq \boldsymbol{\aleph}_{0} \quad(x \in A) .
$$

By Lemma 1 , there is $B(t) \subset A$, for rational $t$, such that $B(t) \subset L(B(u))$ $(t<\mu)$. There are rational numbers $t_{\nu}(\nu<\gamma)$ such that $t_{\mu}>t_{\nu}(\mu<\nu<\gamma)$. We define sets $P_{\nu}(\nu<\gamma)$ as follows. Let $\nu_{0}<\gamma$, and suppose that $P_{\nu} \subset B\left(t_{\nu}\right)\left(\nu<\nu_{0}\right)$. Then we may put, by (35),

$$
B^{\prime}=B\left(t_{\nu_{0}}\right) L_{1}\left(\sum\left[\nu<\nu_{0}\right] P_{\nu}\right) \text {. }
$$

Then, by (29), $\bar{B}^{\prime} \rightarrow\left(\alpha, \omega_{0}\right)^{2} ; \alpha \notin F_{0}\left(B^{\prime}\right) ; \omega_{0} \in F_{1}\left(B^{\prime}\right)$, and there is $P_{\nu_{0}} \subset B^{\prime}$ such that $\left[P_{\nu_{0}}\right]^{2} \subset K_{1}$. This defines $P_{\nu}$ for $\nu<\gamma$. Put $\sum[\nu<\gamma] P_{\nu}$ $=X$. Then $\bar{X}=\omega_{0} \gamma^{*} ;[X]^{2} \subset K_{1}$. But this contradicts (34), and so (a) is proved.

Proof of Theorem 32 (b). Let the hypotheses be satisfied but (b) be false. Then 


$$
\omega_{0}+\omega_{0}^{*} \notin\left[K_{0}\right] ; \quad \gamma \notin\left[K_{1}\right] ; \quad \gamma^{*} \notin\left[K_{1}\right] .
$$

Choose any $A$.

Assumption. $\left|A R_{0}(x)\right| \leqq \boldsymbol{\aleph}_{0}(x \in A)$.

Then, by Lemma 1 , there are sets $B(t) \subset A$, for rational $t$, such that $B(t) \subset L(B(u))(t<u)$. There are rational numbers $t_{\nu}(\nu<\gamma)$ such that $t_{\mu}<t_{\nu}(\mu<\nu<\gamma)$. We define $x_{\nu}(\nu<\gamma)$ as follows. Let $\nu_{0}<\gamma$, and suppose that $x_{\nu} \in B\left(t_{\nu}\right)\left(\nu<\nu_{0}\right)$. Then, by our assumption, there is

$$
x_{\nu_{0}} \in B\left(t_{\nu_{0}}\right)-\sum\left[\nu<\nu_{0}\right] R_{0}\left(x_{\nu}\right) .
$$

Then the set $X=\left\{x_{\nu}: \nu<\gamma\right\}$ satisfies $\bar{X}=\gamma ;[X]^{2} \subset K_{1}$ which is a contradiction against (36). Hence our assumption is false, i.e., given any $A$, there is $x \in A$ such that $\left|A R_{0}(x)\right|=\boldsymbol{\aleph}_{1}$. By symmetry, it follows that there also is $y \in A$ such that $\left|A L_{0}(y)\right|=\boldsymbol{\aleph}_{1}$. By alternate applications of these two results we obtain elements $x_{\nu}, y_{\nu}$ and sets $A_{\nu}, B_{\nu}\left(\nu<\omega_{0}\right)$ such that the following conditions are satisfied.

$x_{0} \in S ; \quad y_{0} \in R_{0}\left(x_{0}\right)=B_{0} ; \quad x_{1} \in B_{0} L_{0}\left(y_{0}\right)=A_{1} ; \quad y_{1} \in A_{1} R_{0}\left(x_{1}\right)=B_{1} ;$

generally, for $\nu<\omega_{0}$,

$$
x_{\nu+1} \in B_{\nu} L_{0}\left(y_{\nu}\right)=A_{\nu+1} ; \quad y_{\nu+1} \in A_{\nu+1} R_{0}\left(x_{\nu+1}\right)=B_{\nu+1} .
$$

Then the set $\sum\left[\nu<\omega_{0}\right]\left\{x_{\nu}, y_{\nu}\right\}=D$ satisfies $\bar{D}=\omega_{0}+\omega_{0}^{*} ;[D]^{2} \subset K_{0}$. This contradiction against (36) completes the proof of Theorem 32.

Proof of Theorem 31, (27). Let $[S]^{2}=K_{0}+K_{1}+K_{2}$,

$$
\alpha \notin\left[K_{\nu}\right]
$$

Our aim is to deduce a contradiction. We shall reduce the general case to more and more special cases. For the sake of convenience of notation we shall use the same notation for the sets in question at each stage.

We put $K_{12}=K_{1}+K_{2}$. The functions $F_{12}, L_{12}, R_{12}$ refer to $K_{12}$ in the same way as the functions $F_{\nu}, L_{\nu}, R_{\nu}$ refer to $K_{\nu}$.

Let $A \subset S$. By Lemma 1 , there are sets $A_{0}, A_{1} \subset A$ such that $A_{0} \subset L\left(A_{1}\right)$. Let $x_{0} \in A_{1}$. Then $\left|A L\left(x_{0}\right)\right|=\boldsymbol{\aleph}_{1}$, and there is $\nu_{0}<3$ such that $\left|A L_{\nu_{0}}\left(x_{0}\right)\right|=\boldsymbol{\aleph}_{1}$. By repeating this argument we find numbers $\nu_{\rho}<3$ and elements $x_{\rho}\left(\rho<\omega_{0}\right)$ such that

$$
\begin{array}{rlrl}
x_{\rho} & \in S L_{\nu_{0}}\left(x_{0}\right) L_{\nu_{1}}\left(x_{1}\right) \cdots L_{\nu_{p-1}}\left(x_{\rho-1}\right), & \\
\left|S L_{\nu_{0}}\left(x_{0}\right) \cdots L_{p_{\rho}}\left(x_{\rho}\right)\right| & =\boldsymbol{N}_{1} \quad\left(\rho<\omega_{0}\right) .
\end{array}
$$

There are $\rho_{0}<\rho_{1}<\cdots \rho_{m-1}<\omega_{0}$ such that $\nu_{\rho_{0}}=\cdots=\nu_{\rho_{m-1}}$. We may assume $\nu_{\rho_{0}}=0$. Put $L_{0}\left(x_{\rho_{0}}, \cdots, x_{\rho_{m-1}}\right)=A_{0}$.

Assumption 1. $\omega_{0} \in F_{0}\left(A_{0}\right)$. 
Then there is $P \subset A_{0}$ such that $[P]^{2} \subset K_{0}$. Then $\alpha \leqq \bar{C} ;[C]^{2} C K_{0}$, where $C=P+\left\{x_{\rho_{\nu}}: \nu<m\right\}$, which contradicts (37). Hence the Assumption 1 is false, and we have $\omega_{0} \notin F_{0}\left(A_{0}\right)$. We may assume that

$$
\omega_{0} \notin\left[K_{0}\right] .
$$

For a later application we remark that in what follows we may replace $S$ by any nondenumerable subset of $S$ without any of the conclusions becoming invalid.

Now let $A \subset S$. Then, by (29), $\bar{A} \rightarrow\left(\omega_{0}, \alpha\right)^{2}$. Also, $\omega_{0} \rightarrow\left(\omega_{0}, \omega_{0}\right)^{2}$. Therefore, by Theorem $16, \bar{A} \rightarrow\left(\omega_{0}, \omega_{0}, \alpha\right)^{2}$. Hence at least one of the following three relations holds.
(i) $\omega_{0} \in F_{0}(A)$,
(ii) $\omega_{0} \in F_{1}(A)$,
(iii) $\alpha \in F_{2}(A)$.

Since (i) and (iii) are false, it follows that

$$
\omega_{0} \in F_{1}(A)
$$

By symmetry,

$$
\omega_{0} \in F_{2}(A) \quad(A \subset S) .
$$

Assumption 2. There are $x_{\nu}, A_{\nu}\left(\nu<\omega_{0}\right)$ such that $x_{0} \in A_{0} ; A_{0} R_{0}\left(x_{0}\right)$ $=A_{1} ; x_{1} \in A_{1} ; A_{1} R_{0}\left(x_{1}\right)=A_{2} ; x_{2} \in A_{2}$, etc.

Then $\left[\left\{x_{0}, x_{1}, \cdots\right\}<\right]^{2} \subset K_{0}$ which contradicts (38). Hence the Assumption 2 is false, and there are $\nu_{0}<\omega_{0} ; x_{\nu} \in S\left(\nu<\nu_{0}\right)$ such that we may put $A=R_{0}\left(x_{0}, \cdots, x_{\nu_{0}-1}\right)$ and we then have $\left|A R_{0}(x)\right| \leqq \boldsymbol{\aleph}_{0}$ $(x \in A)$. We may assume that

$$
\left|R_{0}(x)\right| \leqq \boldsymbol{\aleph}_{0} \quad(x \in S) .
$$

By Lemma 1, there are sets $A, B$ such that $A \subset L(B)$. By (39), there is $P \subset A$ such that $[P]^{2} \subset K_{1}$. For a later application we remark that at this stage we might have applied (40) in place of (39) and in this way could have interchanged the roles of $K_{1}$ and $K_{2}$. By (41), $\left|\sum[x \in P] R_{0}(x)\right| \leqq \boldsymbol{\aleph}_{0}$, and hence $\left|B R_{12}(P)\right|=\boldsymbol{\aleph}_{1}$. Therefore we may assume

$$
[P]^{2} \subset K_{1} ; \quad P \subset L_{12}(S-P) .
$$

Assumption 3. If $Q \subset P ; A \subset S$, then there is $x \in A$ such that

$$
\left|Q L_{1}(x)\right|=\boldsymbol{\aleph}_{0} .
$$

Now we argue as follows. By Lemma 1 , there are sets $A_{\nu} \subset S-P$ such that $A_{\mu} \subset L\left(A_{\nu}\right)\left(\mu<\nu<\omega_{0} m\right)$. We define inductively $x_{\nu}, P_{\nu}\left(\nu<\omega_{0} m\right)$ as follows. There is $x_{0} \in A_{0}$ such that, if $P_{0}=P$, we have $\left|P_{0} L_{1}\left(x_{0}\right)\right|$ $=\boldsymbol{\aleph}_{0}$. Let $0<\nu_{0}<\omega_{0} m$, and suppose that 


$$
\begin{gathered}
x_{\nu} \in A_{\nu} ; \quad P_{\nu} \subset P \\
\left|P_{\nu}-P_{\mu}\right|<\aleph_{0}
\end{gathered}
$$$$
\left(\nu<\nu_{0}\right) \text {, }
$$$$
\left(\mu<\nu<\nu_{0}\right) \text {. }
$$

Then we can write $\left[0, \nu_{0}\right]=\left\{\rho_{\lambda}: \lambda<\omega_{0}\right\}$. We can choose $y_{\lambda}$ such that

$$
y_{\lambda} \in P_{\rho_{0}} P_{\rho_{1}} \cdots P_{\rho_{\lambda}}-\left\{y_{0}, \cdots, y_{\lambda-1}\right\} \quad\left(\lambda<\omega_{0}\right) .
$$

By (41) and Assumption 3, there is $x_{\nu_{0}} \in A_{\nu_{0}}-\sum\left[\nu<\nu_{0}\right] R_{0}\left(x_{\nu}\right)$ such that $\left|\left\{y_{0}, y_{1}, \cdots\right\} L_{1}\left(x_{\nu_{0}}\right)\right|=\boldsymbol{\aleph}_{0}$. We put $P_{\nu_{0}}=\left\{y_{0}, y_{1}, \cdots\right\} L_{1}\left(x_{\nu_{0}}\right)$. Then, if $\nu<\nu_{0}$, there is $\lambda<\omega_{0}$ such that $\nu=\rho_{\lambda}$. Then $\left|P_{\nu_{0}}-P_{\nu}\right|$ $\leqq\left|\left\{y_{0}, y_{1}, \cdots\right\}-P_{p_{\lambda}}\right|<\aleph_{0}$. This completes the definition of $x_{\nu}, P_{\nu}\left(\nu<\omega_{0} m\right)$.

We have $\left|P_{\nu}-P_{\mu}\right|<\boldsymbol{\aleph}_{0}\left(\mu<\nu<\omega_{0} m\right) ; P_{\nu} \subset P L_{1}\left(x_{\nu}\right) \quad\left(\nu<\omega_{0} m\right)$. Put $X=\left\{x_{\nu}: \nu<\omega_{0} m\right\}$. Then, by (11), $[X]^{2} \subset K_{1}+K_{2} ; X=\omega_{0} m \rightarrow(m, \alpha)^{2}$.

Case 1. There is $D=\left\{x_{\mu_{0}}, \cdots, x_{\mu_{m-1}}\right\}<\subset X$ such that $[D]^{2} \subset K_{1}$. Then we put $P^{\prime}=P_{\mu_{m-1}}$ and have, for $\tau<m$,

$$
\left|P^{\prime}-L_{1}\left(x_{\mu_{\tau}}\right)\right| \leqq\left|P^{\prime}-P_{\mu_{\tau}}\right|+\left|P_{\mu_{\tau}}-L_{1}\left(x_{\mu_{\tau}}\right)\right|<\aleph_{0}+0 .
$$

By summing over $\tau$ we obtain $\left|P^{\prime}-L_{1}(D)\right|<\boldsymbol{\aleph}_{0}$. Hence we may put $P^{\prime} L_{1}(D)=Q$, and we then have $\overline{Q+D} \geqq \alpha ;[Q+D]^{2} \subset K_{1}$ which contradicts (37).

Case 2. There is $D \subset X$ such that $\bar{D}=\alpha ;[D]^{2} \subset K_{2}$. This, again, contradicts (37). Hence the Assumption 3 is false, i.e., there are $P^{\prime} \subset P ; A^{\prime} \subset S$ such that

$$
\left|P^{\prime} L_{1}(x)\right|<\aleph_{0} \quad\left(x \in A^{\prime}\right) .
$$

Then there is $A^{\prime \prime} \subset A^{\prime}$ such that the set $P^{\prime} L_{1}(x)$ is constant for $x \in A^{\prime \prime}$. Then there is $P^{\prime \prime}$ such that $P^{\prime} L_{2}(x)=P^{\prime \prime}\left(x \in A^{\prime \prime}\right)$. We have therefore proved that there is $P^{\prime \prime}, A^{\prime \prime}$ such that

$$
\left[P^{\prime \prime}\right]^{2} \subset K_{1} ; \quad P^{\prime \prime} \subset L_{2}\left(A^{\prime \prime}\right) \text {. }
$$

The whole argument from (38) onwards remains valid if $S$ is replaced by any set $A$. Hence it follows from (43) that if $A \subset S$, then there are $P, A^{\prime} \subset A$ such that

$$
[P]^{2} \subset K_{1} ; \quad P \subset L_{2}\left(A^{\prime}\right) .
$$

By Lemma 1 , there are $A_{0}, B_{0}$ such that $A_{0} \subset L\left(B_{0}\right)$. By repeated application of (44) we obtain sets $P_{\nu}, A_{\nu}^{\prime} \quad\left(\nu<\omega_{0}\right)$ such that

$$
\begin{array}{lll}
P_{0}+A_{0}^{\prime} \subset A_{0} ; & {\left[P_{0}\right]^{2} \subset K_{1} ;} & P_{0} \subset L_{2}\left(A_{0}^{\prime}\right), \\
P_{1}+A_{1}^{\prime} \subset A_{0}^{\prime} ; & {\left[P_{1}\right]^{2} \subset K_{1} ;} & P_{1} \subset L_{2}\left(A_{1}^{\prime}\right),
\end{array}
$$

generally, $P_{\nu}+A_{\nu}^{\prime} \subset A_{\nu-1} ;\left[P_{\nu}\right]^{2} \subset K_{1} ; P_{\nu} \subset L_{2}\left(A_{\nu}^{\prime}\right)\left(0<\nu<\omega_{0}\right)$. Then $P_{\nu} \subset A_{0} \subset L\left(B_{0}\right)\left(\nu<\omega_{0}\right)$, 


$$
P_{\mu} \subset L_{2}\left(A_{\mu}^{\prime}\right) \subset L_{2}\left(A_{\nu-1}^{\prime}\right) \subset L_{2}\left(P_{\nu}\right) \quad\left(\mu<\nu<\omega_{0}\right) .
$$

We put $B_{1}=B_{0} R_{12}\left(P_{0}+P_{1}+\cdots\right)$. Then we have the result that there are sets $P_{\nu}, B_{1}\left(\nu<\omega_{0}\right)$ such that

$$
\left\{\begin{array}{lr}
{\left[P_{\nu}\right]^{2} \subset K_{1} ; \quad P_{\nu} \subset L_{12}\left(B_{1}\right)} & \left(\nu<\omega_{0}\right), \\
P_{\mu} \subset L_{-2}\left(P_{\nu}\right) & \left(\mu<\nu<\omega_{0}\right) .
\end{array}\right.
$$

Now let $\nu_{0}<\omega_{0} ; B_{2} \subset B_{1} ; P^{\prime} \subset P_{\nu_{0}}$.

Assumption 4. $\left|P^{\prime} L_{2}(x)\right|<\aleph_{0}\left(x \in B_{2}\right)$.

Then there is $B_{3} \subset B_{2}$ such that the set $D=P^{\prime} L_{2}(x)$ is constant for $x \in B_{3}$. By (39), there is $Q \subset B_{3}$ such that $[Q]^{2} \subset K_{1}$. Then $\left[\left(P^{\prime}-D\right)\right.$ $+Q]^{2} \subset K_{1} ; \omega_{0} 2 \in\left[K_{1}\right]$ which contradicts (37). Hence the Assumption 4 is false, i.e.

$$
\begin{gathered}
\text { if } \nu_{0}<\omega_{0} ; \quad P^{\prime} \subset P_{\nu_{0}} \text {, then } \\
\left|\left\{x: x \in B_{1} ;\left|P^{\prime} L_{2}(x)\right|<\aleph_{0}\right\}\right| \leqq \aleph_{0} .
\end{gathered}
$$

To $B_{1}$ the same argument applies as to $S$, from (38) onwards. The only change we make is that, after (41), we apply (40) instead of (39), so that now the roles of $K_{1}$ and $K_{2}$ are interchanged. We find sets $Q_{\nu}, B_{2} \subset B_{1}$ such that, in analogy to (45), (46), the following statements are true.

$$
\begin{array}{cr}
{\left[Q_{\nu}\right]^{2} \subset K_{2} ; \quad \begin{array}{cr}
Q_{\nu} \subset L_{12}\left(B_{2}\right) \\
Q_{\mu} \subset L_{1}\left(Q_{\nu}\right)
\end{array}} \\
\text { If } \nu_{0}<\omega_{0} ; Q^{\prime} \subset Q_{\nu}, \text { then } & \left(\mu<\nu<\omega_{0}\right), \\
\left|\left\{x: x \in B_{2} ;\left|Q^{\prime} L_{1}(x)\right|<\aleph_{0}\right\}\right| \leqq \boldsymbol{\aleph}_{0} . &
\end{array}
$$

By Lemma 1, there is $B_{\nu}^{\prime} \subset B_{2}\left(\nu<\omega_{0}\right)$ such that $B_{\mu}^{\prime} \subset L\left(B_{\nu}^{\prime}\right)$ $\left(\mu<\nu<\omega_{0}\right)$. Let $P_{\nu}^{\prime} \subset P_{\nu} ; Q_{\nu}^{\prime} \subset Q_{\nu}\left(\nu<\omega_{0}\right)$. Then, by (46), (48), there are at most $\boldsymbol{\aleph}_{0}$ elements $x \in B_{2}$ such that at least one of the relations

$$
\left|P_{\nu}^{\prime} L_{2}(x)\right|<\boldsymbol{\aleph}_{0} ; \quad\left|Q_{\nu}^{\prime} L_{1}(x)\right|<\boldsymbol{\aleph}_{0}
$$

holds. By using this result repeatedly we find elements $x_{\lambda}\left(\lambda<\omega_{0}\right)$ such that, for all $\nu<\omega_{0}$,

$$
\begin{aligned}
x_{0} \in B_{0}^{\prime} ; & \left|P_{\nu} L_{2}\left(x_{0}\right)\right| & =\left|Q_{\nu} L_{1}\left(x_{0}\right)\right|=\boldsymbol{N}_{0}, \\
x_{1} \in B_{1}^{\prime} ; & \left|P_{\nu} L_{2}\left(x_{0}, x_{1}\right)\right| & =\left|Q_{\nu} L_{1}\left(x_{0}, x_{1}\right)\right|=\boldsymbol{\aleph}_{0},
\end{aligned}
$$

generally, $x_{\lambda} \in B_{\lambda}^{\prime}$;

$$
\left|P_{\nu} L_{2}\left(x_{0}, \cdots, x_{\lambda}\right)\right|=\left|Q_{\nu} L_{1}\left(x_{0}, \cdots, x_{\lambda}\right)\right|=\boldsymbol{\aleph}_{0} \quad\left(\nu, \lambda<\omega_{0}\right) .
$$

Since $\omega_{0} \rightarrow\left(\omega_{0}\right)_{3}^{2}$, there is a number $\nu<3$ and a sequence $\lambda_{0}<\lambda_{1}<\cdots$; 
$\lambda_{\rho}<\omega_{0}$, such that $\left[\left\{x_{\lambda_{0}}, x_{\lambda_{1}}, \cdots\right\}<\right]^{2} \subset K_{\nu}$. By (38), $\nu \neq 0$. We can choose $y_{\mu}, z_{\mu}$ such that, for $\mu<\omega_{0}$,

$$
y_{\mu} \in P_{\mu} L_{2}\left(x_{0}, x_{1}, \cdots, x_{\lambda_{m-1}}\right) ; \quad z_{\mu} \in Q_{\mu} L_{1}\left(x_{0}, \cdots, x_{\lambda_{m-1}}\right) .
$$

Put $X=\left\{x_{\lambda_{\rho}}: \rho<m\right\} ; Y=\left\{y_{\mu}: \mu<\omega_{0}\right\} ; Z=\left\{z_{\mu}: \mu<\omega_{0}\right\}$.

Case 1. $\nu=1$. Then $[Z+X]^{2} \subset K_{1} ; \alpha \in\left[K_{1}\right]$.

Case 2. $\nu=2$. Then $[Y+X]^{2} C K_{2} ; \alpha \in\left[K_{2}\right]$. In either case, a contradiction against (37) follows. This proves (27).

Proof of TheOREM 31 , (28). If $[S]^{2}=K_{0}+K_{1}$ and if we put $\gamma=\omega_{0} m$ then we have, by Theorem 32 (a), either (i) $\alpha \in\left[K_{0}\right]$ or (ii) $\beta \leqq \gamma \in\left[K_{1}\right]$ or (iii) $\beta \leqq \omega_{0} m \leqq \omega_{0} \gamma^{*} \notin\left[K_{1}\right]$. This proves (28).

Proof of Theorem 31, (30). Let $[S]^{3}=K_{0}+{ }^{\prime} K_{1}$,

$$
4 \notin\left[K_{0}\right] ; \quad \alpha \notin\left[K_{1}\right] .
$$

We shall deduce a contradiction.

By Theorem 2, there is $n<\omega_{0}$ such that $n \rightarrow(m, m)^{3}$, and $p$ such that

$$
(n-1)(1+m+m(m-1) / 2)<p<\omega_{0} .
$$

By Lemma 1, there is $z_{0} \in S$ such that

$$
\left|L\left(z_{0}\right)\right|,\left|R\left(z_{0}\right)\right|>\boldsymbol{N}_{0}
$$

and then there is $C \subset R\left(z_{0}\right)$ such that $\bar{C}=\eta$.

The following diagram shows the relative position in $S$ and the inclusion relations between the various sets to be considered in the argument that follows. It might be of help to the reader.

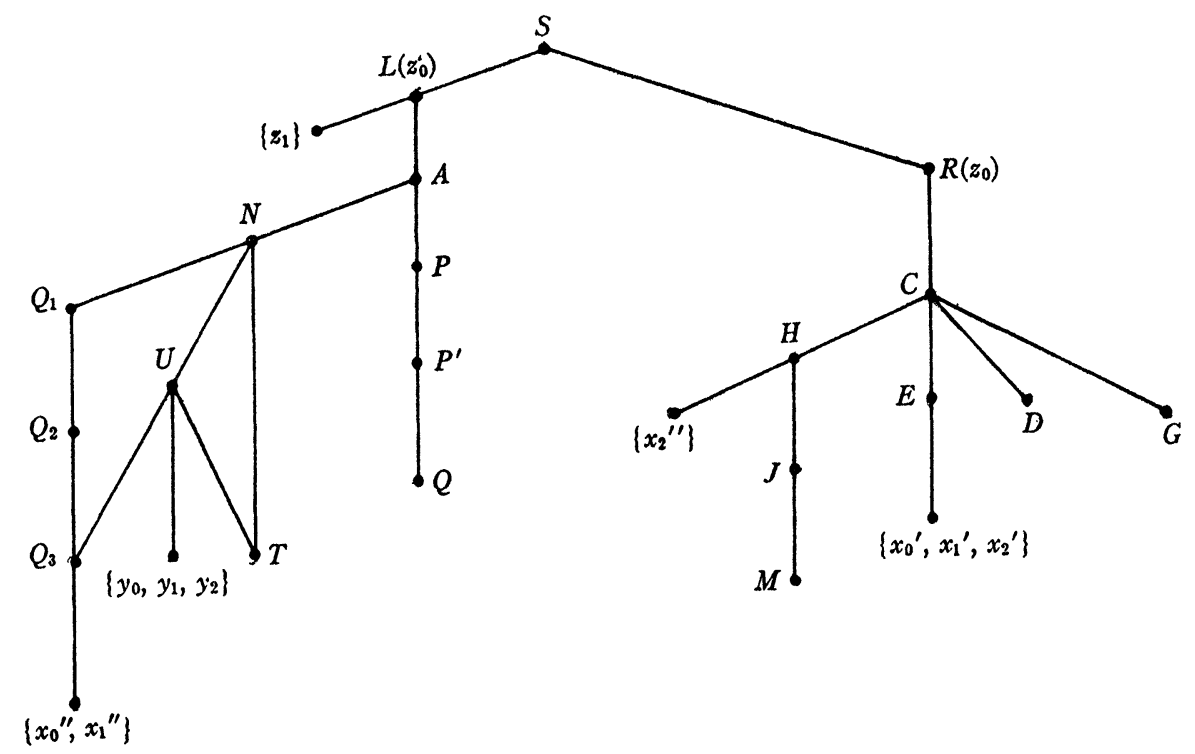


Assumption. If $D \in[C]^{p}$, then $\mid \prod\left[x_{1}, x_{2} \in D\right]\left\{x_{0}: x_{0}<z_{0} ;\left\{x_{0}, x_{1}\right.\right.$, $\left.\left.x_{2}\right\} \notin K_{0}\right\} \mid \leqq \aleph_{0}$. Then there is $z_{1}<z_{0}$ such that

(51) if $D \in[C]^{p}$, then $\left\{z_{1}, x_{1}, x_{2}\right\} \in K_{0} \quad$ for some $x_{1}, x_{2} \in D$.

Then $[C]^{2}=K_{0}^{\prime}+K_{1}^{\prime}$, where

$$
K_{\nu}^{\prime}=\left\{\left\{x_{1}, x_{2}\right\}: x_{1}, x_{2} \in C ;\left\{z_{1}, x_{1}, x_{2}\right\} \in K_{\nu}\right\} \quad(\nu<2) .
$$

By (11), $\bar{C}=\eta \geqq \omega_{0} p \rightarrow\left(\omega_{0}+m, p\right)^{2}$. Hence there are two cases.

Case 1. There is $E \subset C$ such that $\bar{E}=\omega_{0}+m ;[E]^{2} \subset K_{0}^{\prime}$. Then, since, by (49), $\bar{E}=\omega_{0}+m \notin\left[K_{1}\right]$, there are $x_{0}^{\prime}, x_{1}^{\prime}, x_{2}^{\prime} \in E$ such that $\left\{x_{0}^{\prime}, x_{1}^{\prime}, x_{2}^{\prime}\right\} \in K_{0}$. Then $\left[\left\{z_{1}, x_{0}^{\prime}, x_{1}^{\prime}, x_{2}^{\prime}\right\}_{*}\right]^{3} \subset K_{0}$ which contradicts (49).

Case 2. There is $G \in[C]^{p}$ such that $[G]^{2} \subset K_{1}^{\prime}$. Then $\left\{z_{1}, x_{1}, x_{2}\right\} \notin K_{0}$ for all $x_{1}, x_{2} \in G$, which is a contradiction against (51).

It follows that our assumption is false, and that there are $H \in[C]^{p}$ and $A \subset L\left(z_{0}\right)$ such that

$\left\{x_{0}, x_{1}, x_{2}\right\} \notin K_{0} \quad$ for $x_{0} \in A ; x_{1}, x_{2} \in H$

Put

$$
V\left(x_{0}, x_{1}\right)=\left\{x_{2}: x_{2} \in H ;\left\{x_{0}, x_{1}, x_{2}\right\} \in K_{1}\right\} \quad \text { for } x_{0}, x_{1} \in A .
$$

Then $[A]^{2}=K_{0}^{\prime \prime}+^{\prime} K_{1}^{\prime \prime}$, where $K_{0}^{\prime \prime}$ is the set of all $\left\{x_{0}, x_{1}\right\}_{<} \subset A$ such that $\left|V\left(x_{0}, x_{1}\right)\right| \geqq n$. By $(28), \bar{A} \rightarrow\left(\omega_{0}, \omega_{0}+m\right)^{2}$. Hence there are two cases.

Case 1. There is $P \subset A$ such that $[P]^{2} \subset K_{0}^{\prime \prime}$. Then

$$
\begin{aligned}
& \left|V\left(x_{0}, x_{1}\right)\right| \geqq n \quad \text { for }\left\{x_{0}, x_{1}\right\}<\subset P, \\
& {[P]^{2}=\sum[W \subset H] K_{W}^{(3)},}
\end{aligned}
$$

where $K_{W}^{(3)}=\left\{\left\{x_{0}, x_{1}\right\}_{<}: x_{0}, x_{1} \in P ; V\left(x_{0}, x_{1}\right)=W\right\}$. The number $k$ of sets $W$ is finite, and $\omega_{0} \rightarrow\left(\omega_{0}\right)_{k}^{2}$, by Theorem 1 . Hence there are $P^{\prime} \subset P ; J \subset H$ such that $\left[P^{\prime}\right]^{2} \subset K_{J}^{(3)}$,

$$
V\left(x_{0}, x_{1}\right)=J \quad \text { for }\left\{x_{0}, x_{1}\right\}<\subset P^{\prime} .
$$

Then

$$
\left\{x_{0}, x_{1}, x_{2}\right\} \in K_{1}|J| \geqq n, \quad \text { for }\left\{x_{0}, x_{1}\right\}<\subset P^{\prime} ; x_{2} \in J .
$$

Since $\left[P^{\prime}\right]^{3} \subset K_{0}+K_{1}$ and, by Theorem $1, \omega_{0} \rightarrow\left(\omega_{0}, \omega_{0}\right)^{3}$, there are $Q \subset P^{\prime} ; \nu<2$ such that $[Q]^{3} \subset K_{\nu}$. By (49), $\omega_{0} \notin\left[K_{0}\right]$. Hence $\nu=1$; $[Q]^{3} \subset K_{1}$.

Furthermore, $[J]^{3} \subset K_{0}+K_{1} ; \bar{J}=n \rightarrow(m, m)^{3}$. Hence there are 
$M \in[J]^{m} ; \rho<2$ such that $[M]^{3} \subset K_{\rho}$. Since $m \geqq 4 \notin\left[K_{0}\right]$, we have $\rho=1$. Then, in view of $Q \subset P^{\prime} \subset P \subset A ; M \subset J \subset H$,

$$
[Q+M]^{3} \subset K_{1} ; \quad \omega_{0}+m=\overline{Q+M} \in\left[K_{1}\right]
$$

which contradicts (49).

Case 2. There is $N \subset A$ such that $\bar{N}=\omega_{0}+m ;[N]^{2} \subset K_{1}^{\prime \prime}$. Then

$$
\left|V\left(x_{0}, x_{1}\right)\right| \leqq n-1 \quad \text { for } x_{0}, x_{1} \in N .
$$

Then

$$
N=Q_{1}+T ; \quad Q_{1} \subset L(T) ; \quad|T|=m .
$$

We have $\left[Q_{1}\right]^{2}=\sum^{\prime}\left[\kappa<k_{1}\right] K_{\kappa}^{(1)}$, where $K_{\kappa}^{(1)} \neq 0 \quad\left(\kappa<k_{1}\right)$, and two elements $Z_{0}, Z_{1}$ of $\left[Q_{1}\right]^{2}$ belong to the same $K_{\kappa}^{(1)}$ if, and only if, for every $x_{2} \in H$, the sets $Z_{0}+\left\{x_{2}\right\}$ and $Z_{1}+\left\{x_{2}\right\}$ belong to the same class $K_{\nu}$. Then $k_{1}<\omega_{0}$ and, by $\omega_{0} \rightarrow\left(\omega_{0}\right)_{k_{1}}^{2}$, there are $Q_{2} \subset Q_{1} ; k_{2}<k_{1}$ such that $\left[Q_{2}\right]^{2} \subset K_{k_{2}}^{(1)}$. This means that, for some $\rho\left(x_{2}\right)<2$,

$$
\left\{x_{0}, x_{1}, x_{2}\right\} \in K_{\rho\left(x_{2}\right)} \quad \text { for }\left\{x_{0}, x_{1}\right\}<\subset Q_{2} ; x_{2} \in H .
$$

Similarly, we have

$$
Q_{2}=\sum^{\prime}\left[\kappa<k_{2}\right] K_{\kappa}^{(2)}, \text { where } K_{\kappa}^{(2)} \neq 0 \quad\left(\kappa<k_{2}\right),
$$

and two elements $x_{00}$ and $x_{01}$ of $Q_{2}$ belong to the same $K_{k}^{(2)}$ if, and only if, for every $x_{1} \in T ; x_{2} \in H$, the two sets $\left\{x_{00}, x_{1}, x_{2}\right\}$ and $\left\{x_{01}\right.$, $\left.x_{1}, x_{2}\right\}$ belong to the same class $K_{\nu}$. Then $k_{2}<\omega_{0}$ and, by $\omega_{0} \rightarrow\left(\omega_{0}\right)_{k_{2}}^{1}$, there are $Q_{3} \subset Q_{2} ; \kappa_{3}<k_{2}$ such that $Q_{3} \subset K_{\kappa_{3}}^{(2)}$. This means that, for some $\sigma\left(x_{1}, x_{2}\right)<2$,

$$
\left\{x_{0}, x_{1}, x_{2}\right\} \in K_{\sigma\left(x_{1}, x_{2}\right)} \quad \text { for } x_{0} \in Q_{3} ; x_{1} \in T ; x_{2} \in H .
$$

Put $U=Q_{3}+T$, and choose $\left\{x_{0}^{\prime \prime}, x_{1}^{\prime \prime}\right\}_{<} \subset Q_{3}$.

Consider any $x_{2} \in H$ such that there are $x_{0}, x_{1} \in U$ satisfying $\left\{x_{0}, x_{1}, x_{2}\right\}<\in K_{1}$. If $x_{0}, x_{1} \in Q_{3}$, then $\rho\left(x_{2}\right)=1 ;\left\{x_{0}^{\prime \prime}, x_{1}^{\prime \prime}, x_{2}\right\} \in K_{1}$; $x_{2} \in V\left(x_{0}^{\prime \prime}, x_{1}^{\prime \prime}\right)$. If $x_{0} \in Q_{3} ; x_{1} \in T$, then $\sigma\left(x_{1}, x_{2}\right)=1 ;\left\{x_{0}^{\prime \prime}, x_{1}, x_{2}\right\} \in K_{1}$; $x_{2} \in V\left(x_{0}^{\prime \prime}, x_{1}\right)$. If $x_{0}, x_{1} \in T$, then $\left\{x_{0}, x_{1}, x_{2}\right\} \in K_{1} ; x_{2} \in V\left(x_{0}, x_{1}\right)$. Hence, in any case,

$$
x_{2} \in V\left(x_{0}^{\prime \prime}, x_{1}^{\prime \prime}\right)+\sum[x \in T) V\left(x_{0}^{\prime \prime}, x\right)+\sum[\{x, y\}<C T] V(x, y)
$$

and therefore, in view of the definition of $x_{2}$ and the relations $|T|=m$ and (50),

$$
\begin{aligned}
& \left|\sum\left[x_{0}, x_{1} \in U\right]\left\{x_{2}: x_{2} \in H ;\left\{x_{0}, x_{1}, x_{2}\right\}_{<} \in K_{1}\right\}\right| \\
& \quad \leqq(n-1)+(n-1)\left(\begin{array}{c}
m \\
1
\end{array}\right)+(n-1)\left(\begin{array}{c}
m \\
2
\end{array}\right)<p=|H| .
\end{aligned}
$$


We deduce the existence of $x_{2}^{\prime \prime} \in H$ such that

$$
\left\{x_{0}, x_{1}, x_{2}^{\prime \prime}\right\} \in K_{1} \quad \text { for all } x_{0}, x_{1} \in U .
$$

Since $\bar{U}=\omega_{0}+m \notin\left[K_{1}\right]$, there are $y_{0}, y_{1}, y_{2} \in U$ such that $\left\{y_{0}, y_{1}, y_{2}\right\}$ $\in K_{0}$. But then $\left[\left\{y_{0}, y_{1}, y_{2}, x_{2}^{\prime \prime}\right\}_{\neq}\right]^{3} \subset K_{0}$ which contradicts (49). This proves (30) and thus completes the proof of Theorems 31 and 32 .

Theorem 33. Let $\alpha<\omega_{0} 2$. Then $\omega_{1} \rightarrow(\alpha, \alpha)^{2}$.

Proof. Let $\bar{S}=\omega_{1} ;[S]^{2}=K_{0}+^{\prime} K_{1} ; 2 \leqq m<\omega_{0} ; \alpha \leqq \omega_{0}+m$,

$$
\alpha \in\left[K_{\nu}\right] \quad(\nu<2) .
$$

We have to deduce a contradiction. Let the conventions concerning the use of the letters $A, B, P, Q$ be the same as in the proofs of Theorems 31 and 32. Choose any $P$.

Assumption. Let $[P]^{2} \subset K_{0}$. Suppose that, if $P^{\prime} \subset P$, then there is $A$ such that

$$
\left|P^{\prime} L_{0}(x)\right|=\mathbf{\aleph}_{0} \quad(x \in A) .
$$

Then we define $x_{\nu}, P_{\nu}\left(\nu<\omega_{1}\right)$ as follows. There is $x_{0}$ such that $\left|P L_{0}\left(x_{0}\right)\right|=\aleph_{0}$. Put $P_{0}=P L_{0}\left(x_{0}\right)$. Now let $0<\nu_{0}<\omega_{1}$, and suppose that $x_{\nu} \in S ; P_{\nu} \subset P L_{0}\left(x_{\nu}\right)\left(\nu<\nu_{0}\right)$;

$$
\left|P_{\nu}-P_{\mu}\right|<\aleph_{0} \quad\left(\mu<\nu<\nu_{0}\right) .
$$

Then we can write $\left[0, \nu_{0}\right]=\left\{\mu_{\lambda}: \lambda<\omega_{0}\right\}$. We can choose elements $y_{\lambda}\left(\lambda<\omega_{0}\right)$ such that $y_{\lambda} \in P_{\mu_{0}} P_{\mu_{1}} \cdots P_{\mu_{\lambda}}-\left\{y_{\rho}: \rho<\lambda\right\} \quad\left(\lambda<\omega_{0}\right)$. Put $P^{\prime}=\left\{y_{\lambda}: \lambda<\omega_{0}\right\}$. Then, by our assumption, there is $A$ such that $\left|P^{\prime} L_{0}(x)\right|=\boldsymbol{\aleph}_{0}(x \in A)$. We can choose

$$
x_{\nu_{0}} \in A-\sum\left[\nu<\nu_{0}\right]\left(\left\{x_{\nu}\right\}+L\left(x_{\nu}\right)\right) .
$$

We put $P_{\nu_{0}}=P^{\prime} L_{0}\left(x_{\nu_{0}}\right)$. If, now, $\nu_{1}<\nu_{0}$, then there is $\lambda<\omega_{0}$ such that $\nu_{1}=\mu_{\lambda}$. Then

$$
\left|P_{\nu_{0}}-P_{\nu_{1}}\right| \leqq\left|\left\{y_{0}, y_{1}, \cdots\right\}-P_{\mu_{\lambda}}\right|<\boldsymbol{\aleph}_{0} .
$$

Also, $P_{\nu_{0}} \subset P L_{0}\left(x_{v_{0}}\right)$. This completes the inductive definition of $x_{\nu}, P_{\nu}\left(\nu<\omega_{1}\right)$ such that

$$
P_{\mu} \subset P L_{0}\left(x_{\mu}\right) ; \quad\left|P_{\nu}-P_{\mu}\right|<\aleph_{0} \quad\left(\mu<\nu<\omega_{1}\right) .
$$

Put $X=\left\{x_{\nu}: \nu<\omega_{1}\right\}$. Then, by Theorem 23, $\bar{X}=\omega_{1}>\omega_{0} m \rightarrow\left(m, \omega_{0}\right.$ $+m)^{2}$. Since, by (52), $\omega_{0}+m \in F_{1}(X)$, we have $m \in F_{0}(X)$, and there is $D \in[X]^{m}$ such that $[D]^{2} \subset K_{0}$. Let $x_{\rho}=\max [x \in D] x$. Then, for any $x_{\nu} \in D,\left|P_{\rho}-L_{0}\left(x_{\nu}\right)\right| \leqq\left|P_{\rho}-P_{\nu}\right|+\left|P_{\nu}-L_{0}\left(x_{\nu}\right)\right|<\boldsymbol{\aleph}_{0}$. Hence we may put $Q=P_{\rho} L_{0}(D)$, and then we have $\overline{Q+D}=\omega_{0}+m \geqq \alpha ;[Q+D]^{2}$ 
$C K_{0}$. This is a contradiction against (52). Therefore our assumption is false.

Now let $A \subset S$. Then, by Theorem 3, $|A|=\boldsymbol{\aleph}_{1} \rightarrow\left(\boldsymbol{\aleph}_{0}, \boldsymbol{\aleph}_{1}\right)^{2}$ and hence, by Theorem 14, $\bar{A}=\omega_{1} \rightarrow\left(\omega_{0}, \omega_{1}\right)^{2}$. Since $\omega_{1} \in F_{1}(A)$, we conclude that $\omega_{0} \in F_{0}(A)$, so that there is $P \subset A$ such that $[P]^{2} \subset K_{0}$. As the assumption made above is false, there is $P^{\prime} \subset P$ such that there are at most $\boldsymbol{\aleph}_{0}$ elements $x$ such that $\left|P^{\prime} L_{0}(x)\right|=\boldsymbol{\aleph}_{0}$. Then there is $A^{\prime} \subset A$ such that $\left|P^{\prime} L_{0}(x)\right|<\boldsymbol{\aleph}_{0}\left(x \in A^{\prime}\right)$. Hence there are at most $\aleph_{0}$ distinct sets $P^{\prime} L_{0}(x)$ for varying values $x \in A^{\prime}$, and there is $A^{\prime \prime} \subset A^{\prime}$ and $E$ such that

$$
P^{\prime} L_{0}(x)=E \quad\left(x \in A^{\prime \prime}\right) .
$$

Since $|E|<\boldsymbol{\aleph}_{0}$, we may put $P^{\prime \prime}=P^{\prime}-E$, and since $\bar{A}^{\prime \prime}=\omega_{1} ; \bar{P}^{\prime \prime}=\omega_{0}$, we may put $A^{\prime \prime \prime}=A^{\prime \prime} R\left(P^{\prime \prime}\right)$. Now let $x \in A^{\prime \prime \prime} ; y \in P^{\prime \prime}$. Then

$$
x \in A^{\prime \prime \prime} \subset A^{\prime \prime} ; \quad y \notin E=P^{\prime} L_{0}(x) ; \quad y \notin L_{0}(x) .
$$

Also,

$$
x \in A^{\prime \prime \prime} \subset R\left(P^{\prime \prime}\right) \subset R(y) ; x>y .
$$

Hence

$$
y \in L_{1}(x) ; \quad P^{\prime \prime} \subset L_{1}\left(A^{\prime \prime \prime}\right) .
$$

So far we have proved that, given any $A$, there are sets $P^{\prime \prime \prime}, A^{\prime \prime} \subset A$ such that $P^{\prime \prime} \subset L_{1}\left(A^{\prime \prime \prime}\right) ;\left[P^{\prime \prime}\right]^{2} \subset K_{0}$, and, moreover, there are at most $\boldsymbol{\aleph}_{0}$ elements $x$ such that $\left|P^{\prime \prime} L_{0}(x)\right|=\boldsymbol{\aleph}_{0}$.

By applying the last result repeatedly, starting with $A=S$, we obtain sets $P_{\nu}\left(\nu<\omega_{0}\right)$ such that

$$
\left[P_{\mu}\right]^{2} \subset K_{0} ; \quad P_{\mu} \subset L_{1}\left(P_{\nu}\right) \quad\left(\mu<\nu<\omega_{0}\right) .
$$

There is $Q_{v}$ such that

$$
\left|P_{\nu} L_{0}(x)\right|<\boldsymbol{\aleph}_{0} \quad\left(\nu<\omega_{0} ; x \in S-Q_{\nu}\right) .
$$

We can choose $B \subset S-\sum\left[\nu<\omega_{0}\right] Q_{\nu}$ such that $P_{\nu} \subset L(B) \quad\left(\nu<\omega_{0}\right)$. Then, by Theorem $23, \bar{B}=\omega_{1}>\omega_{0} m \rightarrow\left(\omega_{0}+m, m\right)^{2}$;

$$
\omega_{0}+m \in F_{0}(B) ; \quad m \in F_{1}(B),
$$

and there is $D \in[B]^{m}$ such that $[D]^{2} \subset K_{1}$. Then, for every $\nu<\omega_{0}$, $\left|\sum[x \in D] P_{\nu} L_{0}(x)\right|<\boldsymbol{\aleph}_{0}$. Therefore we can choose $y_{v} \in P_{\nu} L_{1}(D)$ $\left(\nu<\omega_{0}\right)$. If we put $Q=\left\{y_{\nu}: \nu<\omega_{0}\right\}$ then $\overline{Q+D}=\omega_{0}+m \geqq \alpha ;[Q+D]^{2}$ $\subset K_{1}$. This contradiction against (52) completes the proof of Theorem 33.

The next theorem, while perhaps appearing to be of a rather special 
and complicated nature, is of interest in that it implies Theorem 7 (i) and Theorem 8. It may well be capable of further worthwhile applications.

Theorem 34. Let $\alpha, \beta, \gamma$ be ordinals, and $\alpha \rightarrow(\beta, \gamma)^{2}$. Then there are ordinals $\alpha_{\lambda}\left(\lambda<\beta^{-}\right)$such that, if

then

$$
\left|k_{\mu}\right|=\prod[\lambda<\mu]\left|\alpha_{\lambda}\right| \quad\left(\mu<\beta^{-}\right),
$$

$$
\alpha \rightarrow\left(\alpha_{0}+1, \alpha_{1}+1, \cdots\right)_{\beta^{-} ;}^{1} ; \quad \alpha_{\lambda} \rightarrow(\gamma)_{k_{\lambda}}^{1} \quad\left(\lambda<\beta^{-}\right) .
$$

We begin by deducing (i) of Theorem 7 or, rather, a slightly stronger proposition, from Theorem 34 .

Corollary 1. Let $m$ and $n$ be such that $\boldsymbol{\aleph}_{n}^{d} \leqq \boldsymbol{\aleph}_{n}\left(d<\boldsymbol{\aleph}_{m}\right)$. Then $\omega_{n+1} \rightarrow\left(\omega_{m}+1, \omega_{n+1}\right)^{2}$.

This implies, a fortiori, $\omega_{n+1} \rightarrow\left(\omega_{m}, \omega_{n+1}\right)^{2}$ which, in its turn, by Theorem 14, is equivalent to Theorem 7 (i).

Deduction of Corollary 1 from Theorem 34. Let us suppose that $\omega_{n+1} \rightarrow\left(\omega_{m}+1, \omega_{n+1}\right)^{2}$. Then, by Theorem 34 , there are ordinals $\alpha_{\lambda}, k_{\lambda}$ such that $\left|k_{\mu}\right|=\prod[\lambda<\mu]\left|\alpha_{\lambda}\right|\left(\mu<\omega_{m}\right)$;

$$
\begin{aligned}
\omega_{n+1} \rightarrow\left(\alpha_{0}+1, \alpha_{1}+1, \cdots\right)_{\omega_{m}}^{1}, & \\
\alpha_{\mu} \rightarrow\left(\omega_{n+1}\right)_{k_{\mu}}^{1} & \left(\mu<\omega_{m}\right) .
\end{aligned}
$$

Then, for $\lambda<\omega_{m}$,

$$
\alpha_{\lambda}<\omega_{n+1} .
$$

For, let $\mu<\omega_{m}$, and suppose that (55) holds for $\lambda<\mu$. Then, using $|\mu|<\boldsymbol{\aleph}_{m}$ and the hypothesis, we find that $\left|k_{\mu}\right| \leqq \boldsymbol{N}_{n}^{|\mu|} \leqq \boldsymbol{\aleph}_{n}$. Now, by (54), we can write $\left|\alpha_{\mu}\right|=\sum\left[\nu<k_{\mu}\right]\left|\rho_{\nu}\right|$, where $\rho_{\nu}<\omega_{n+1} \quad\left(\nu<k_{\mu}\right)$. Hence $\left|\alpha_{\mu}\right| \leqq \boldsymbol{\aleph}_{n}\left|k_{\mu}\right| \leqq \boldsymbol{\aleph}_{n}$. This proves (55) for all $\lambda<\omega_{m}$. Now, by (53) and the obvious relation $m \leqq n$,

$$
\left|\omega_{n+1}\right| \leqq \sum\left[\lambda<\omega_{m}\right]\left|\alpha_{\lambda}\right| \leqq \boldsymbol{\aleph}_{n} \boldsymbol{\aleph}_{m}=\boldsymbol{\aleph}_{n}
$$

which is the required contradiction.

CoRollary 2. Let $\boldsymbol{\aleph}_{n}^{\prime}=\boldsymbol{\aleph}_{n} ; 2^{\boldsymbol{\aleph}_{\nu}}<\boldsymbol{\aleph}_{n}$ for all $\nu<n$. Then

$$
\omega_{n} \rightarrow\left(\beta, \omega_{n}\right)^{2} \quad\left(\beta<\omega_{n}\right) .
$$

By Theorem 14, this proposition implies Theorem 8.

Deduction of Corollary 2 from Theorem 34. Let $\beta<\omega_{n}$. Suppose that $\omega_{n} \rightarrow\left(\beta, \omega_{n}\right)^{2}$. Then, by Theorem 34, there are ordinals $\alpha_{\lambda}, k_{\lambda}$ such that $\left|k_{\mu}\right|=\prod[\lambda<\mu]\left|\alpha_{\lambda}\right|\left(\mu<\beta^{-}\right)$; 


$$
\omega_{n} \rightarrow\left(\alpha_{0}+1, \cdots\right)_{\beta^{-} ;}^{1} ; \quad \alpha_{\mu} \rightarrow\left(\omega_{n}\right)_{k_{\mu}}^{1} \quad\left(\mu<\beta^{-}\right) .
$$

Let us assume that, for some $\mu<\beta^{-}$, we have $\alpha_{\lambda}<\omega_{n}(\lambda<\mu)$. Then, putting $\quad d=\sum[\lambda<\mu]\left|\alpha_{\lambda}\right|, \quad d<\aleph_{n} ; \quad\left|k_{\mu}\right| \leqq d^{|\mu|} \leqq 2^{d|\mu|}<\boldsymbol{\aleph}_{n} ; \quad\left|\alpha_{\mu}\right|$ $=\sum\left[\nu<k_{\mu}\right]\left|\rho_{\nu}\right|$, where $\rho_{\nu}<\omega_{n}$. Then $\left|\alpha_{\mu}\right|<\boldsymbol{N}_{n}$. This proves, by induction, that $\alpha_{\lambda}<\omega_{n}\left(\lambda<\beta^{-}\right)$. Now, $\left|\omega_{n}\right|=\sum\left[\lambda<\beta^{-}\right]\left|\sigma_{\lambda}\right|$, where $\left|\sigma_{\lambda}\right| \leqq\left|\alpha_{\lambda}\right|$. Therefore $\left|\omega_{n}\right|<\aleph_{n}$ which is the required contradiction.

The proof of Theorem 34 depends on a lemma.

LEMma 2. Let $T$ be a well ordered set, and $[T]^{2}=K_{0}+K_{1}$. Then there $i^{8}$ a set $B=B(T) \subset T$ which has the following properties. We have $[B]^{2} \subset K_{1}$. If $x \in T-B$, then there is $y \in B$ such that $\{y, x\}<\in K_{0}$.

Proof. We may assume $T \neq 0$. Choose $l$ such that $|l|>|T|$. We define, inductively, $y_{\lambda}(\lambda<l)$ as follows. Let $\mu<l ; y_{\lambda} \in T(\lambda<\mu)$.

Case 1 . There is $y \in T$ such that $\left\{y_{\lambda}, y\right\} \in K_{1}(\lambda<\mu)$. Then we take as $y_{\mu}$ the first element $y$ of this kind.

Case 2. If $y \in T$, then there is $\lambda<\mu$ such that $\left\{y_{\lambda}, y\right\} \in K_{1}$. Then we have $\mu>0$. We put $y_{\mu}=y_{0}$.

Let $B=\left\{y_{\lambda}: \lambda<l\right\}$. Then there is $m<l$ such that

$$
B=\left\{y_{\lambda}: \lambda<m\right\} ; \quad\left\{y_{\lambda}, y_{\mu}\right\}_{<} \in K_{1} \quad(\lambda<\mu<m) .
$$

For, $m$ is the least $\mu$ such that $0<\mu<l ; y_{\mu}=y_{0}$. We have $[B]^{2} \subset K_{1}$. Now let $x \in T-B$. Then, by definition of $y_{m}$, there is a least $\mu<m$ such that $\left\{y_{\mu}, x\right\} \in K_{0}$. Then $\left\{y_{\lambda}, x\right\} \in K_{1}(\lambda<\mu)$ and hence, by definition of $y_{\mu}, x>y_{\mu}$. This proves Lemma 2 .

Proof of Theorem 34 . There is an ordered set $S$ such that

$$
\bar{S}=\alpha ; \quad[S]^{2}=K_{0}+K_{1} ; \quad \beta \in\left[K_{0}\right] ; \quad \gamma \notin\left[K_{1}\right] .
$$

We choose an ordinal $\rho$ such that $|\rho|>|\alpha|$. Let $x \in S$. We define $f_{\mu}(x)(\mu<\rho)$ as follows. Let $\nu<\rho$, and suppose that

$$
\begin{aligned}
& f_{\mu}(x) \in S(\mu<\nu), \\
&\left\{f_{\mu}(x), x\right\}_{<} \in K_{0} \text { if } \mu<\nu ; f_{\mu}(x) \neq x .
\end{aligned}
$$

Then we define $f_{\nu}(x)$ by the following rule. If $f_{\mu}(x)=x$ for some $\mu<\nu$, then put $f_{\nu}(x)=x$. Now let $f_{\mu}(x) \neq x(\mu<\nu)$. Let $T$ be the set of all $y \in S$ such that $\left\{f_{\mu}(x), y\right\}<\in K_{0}(\mu<\nu)$. Then $x \in T$. Let $B=B(T)$ be the set given in Lemma 2. Then $B \subset T ;[B]^{2} \subset K_{1} ; \bar{B}<\gamma$. If $x \in B$, then put $f_{\nu}(x)=x$. Now let $x \in B$. Then, by Lemma 2 , there is a first element $z \in B$ such that $\{z, x\}<\in K_{0}$. We put $f_{\nu}(x)=z$. We have now defined $f_{\nu}(x)$ for $\nu<\rho ; x \in S$, and we have

${ }^{8}$ In fact, there is exactly one such set. 


$$
\begin{aligned}
\left\{f_{\mu}(x), f_{\nu}(x)\right\}_{<} \in K_{0} & & \left(\mu<\nu<\rho ; f_{\mu}(x) \neq x\right) ; \\
f_{\nu}(x) \leqq x & & (\nu<\rho ; x \in S) .
\end{aligned}
$$

If, for some $x, f_{\nu}(x)<x(\nu<\rho)$, then the contradiction

$$
|\rho|=\left|\left\{f_{\nu}(x): \nu<\rho\right\}\right| \leqq|S|=|\alpha|
$$

follows. Hence, given $x \in S$, there is $\sigma(x)<\rho$ such that

$$
f_{\nu}(x)<x \quad(\nu<\sigma(x)) ; \quad f_{\sigma(x)}(x)=x \quad(x \in S) .
$$

Then, for fixed $x,\left[\left\{f_{v}(x): \nu \leqq \sigma(x)\right\}\right]^{2} \subset K_{0}$,

$$
\sigma(x)+1<\beta ; \quad \sigma(x)<\beta^{-} .
$$

Put $M_{\nu}=\left\{f_{\nu}(x): \sigma(x) \geqq \nu\right\}(\nu<\rho)$. Then $M_{0} \subset B(S) ; \bar{M}_{0}<\gamma$;

$$
S=\sum\left[\nu<\beta^{-}\right] M_{\nu} ; \quad \alpha \leftrightarrow\left(\bar{M}_{0}+1, \bar{M}_{1}+1, \cdots\right)_{\beta^{-}}^{1} .
$$

Let $0<\nu<\beta^{-}$. Then

$$
M_{\nu}=\sum\left[y_{\mu} \in M_{\mu} \text { for } \mu<\nu\right]\left\{f_{\nu}(x): \sigma(x) \geqq \nu ; f_{\lambda}(x)=y_{\lambda} \text { for } \lambda<\nu\right\} \text {. }
$$

Now, for every choice of $y_{\mu} \in M_{\mu}(\mu<\nu)$, the corresponding term in the last sum is a set contained in some set of the form $B(T)$. In order to see this, consider an element $x$ such that $\sigma(x) \geqq \nu ; f_{\mu}(x)=y_{\mu}(\mu<\nu)$. We shall have $f_{\mu}(x)<x(\mu<\sigma(x))$ and hence $f_{\mu}(x)<x(\mu<\nu)$. Let $T$ be the set used above in the inductive definition of $f_{\nu}(x)$, i.e. the set of all $y \in S$ such that $\left\{f_{\mu}(x), y\right\}<\in K_{0}(\mu<\nu)$. Then, by definition of $f_{\nu}(x), x \in T$ and either

$$
x \in B(T) ; \quad f_{\nu}(x)=x \in B(T)
$$

or

$$
x \notin B(T) ; \quad f_{\nu}(x)=z \in B(T) .
$$

In either case, $f_{\nu}(x) \in B(T)$. In fact, the set $T$ does not depend on $x$ since $T$ is the set of all $y \in S$ such that

$$
\left\{y_{\mu}, y\right\}<\in K_{0}
$$

All this proves that, given $y_{\mu} \in M_{\mu}(\mu<\nu)$, there is $T$ such that, whenever

$$
x \in S ; \quad \sigma(x) \geqq \nu ; \quad f_{\mu}(x)=y_{\mu} \quad(\mu<\nu),
$$

then

$$
f_{\nu}(x) \in B(T) .
$$

By definition of $B(T)$, we have $[B(T)]^{2} C K_{1}$ and therefore $\overline{B(T)}<\gamma$. 
Hence $M_{\nu}$ is a sum of $\Pi[\mu<\nu]\left|M_{\mu}\right|$ sets each of type less than $\gamma$. This shows that $\bar{M}_{\nu} \rightarrow(\gamma)_{k_{\nu}}^{1}$, when $k_{\nu}$ is any ordinal such that $\left|k_{\nu}\right|$ $=\prod[\mu<\nu]\left|M_{\mu}\right|$. It now follows that the conclusion of Theorem 34 holds for $\alpha_{\lambda}=\bar{M}_{\lambda}\left(\lambda<\beta^{-}\right)$.

THEOREM 35. Suppose that $\beta \geqq r \geqq 3 ; \beta, \beta^{*} \neq \alpha ; s>(r-1)^{2}$. Then, for any type $\phi$ such that $|\phi|=|\alpha|$,

$$
\phi \rightarrow(s, \beta)^{r} \text {. }
$$

CoROllaRy. If $r \geqq 3 ; s>(r-1)^{2}$, then

$$
\begin{gathered}
\eta \nrightarrow\left(s, \omega_{0}+1\right)^{r}, \\
\phi \mapsto\left(s, \omega_{1}\right)^{r},
\end{gathered}
$$

where $\phi$ is any type such that $|\phi|=|\lambda|$.

The negative results (57) and (58) are not too far from the ultimate truth as is seen by comparing them with the following positive results. By Theorem 1,

$$
\omega_{0} \rightarrow\left(\omega_{0}, \omega_{0}, \cdots, \omega_{0}\right)_{k}^{r} \quad\left(k<\omega_{0}\right) .
$$

By Theorem 31,

$$
\phi \rightarrow\left(\omega_{0}, \gamma\right)^{2} \quad\left(\gamma<\omega_{1}\right),
$$

where $\phi$ is any type such that $|\phi|>\boldsymbol{N}_{0} ; \omega_{1}, \omega_{1}^{*} \ddagger \phi$.

Proof of Theorem 35. The corollary follows by applying the theorem to the following two cases.

(i) $\beta=\omega_{0}+1 ; \alpha=\omega_{0} ; \phi=\eta$,

(ii) $\beta=\omega_{1} ; \alpha=\lambda$.

The proof of the theorem depends on the following lemma due to Erdös and Szekeres [7]. Throughout, we put

$$
s=(r-1)^{2}+1 .
$$

Lemma 3. If $S$ is an ordered set, $r>0$, and if $z(\sigma) \in S(\sigma<s)$, then there is $\left\{\sigma_{0}, \sigma_{1}, \cdots, \sigma_{r-1}\right\}<\subset[0, s]$ such that either

$$
z\left(\sigma_{\rho}\right) \leqq z\left(\sigma_{\rho+1}\right) \quad(\rho+1<r)
$$

or

$$
z\left(\sigma_{\rho}\right) \geqq z\left(\sigma_{\rho+1}\right) \quad(\rho+1<r) .
$$

We now prove the theorem. Let $\bar{S}_{<}=\phi ; \bar{S}_{<<}=\alpha$. Then

$$
[S]^{r}=K_{0}+{ }^{\prime} K_{1} ; \quad K_{1}=K_{10}+K_{11},
$$


where

$$
\begin{aligned}
& K_{10}=\left\{\left\{x_{0}, \cdots, x_{r-1}\right\}_{<:}:\left\{x_{0}, \cdots, x_{r-1}\right\}_{<<} \subset S\right\}, \\
& K_{11}=\left\{\left\{x_{0}, \cdots, x_{r-1}\right\}_{<:}:\left\{x_{0}, \cdots, x_{r-1}\right\}_{\gg>\subset S\} .}\right.
\end{aligned}
$$

Case 1. There is $A \in[S]^{s}$ such that $[A]^{r} \subset K_{0}$. Then, if $A=\{z(\sigma)$ : $\sigma<s\}$, an application of Lemma 3 shows the existence of $B \in[A]^{r}$ such that $B \in K_{1}$, which is a contradiction.

Case 2. There is $A \subset S$ such that $\bar{A}_{<}=\beta ;[A]^{r} \subset K_{1}$. We shall prove that one of the two relations

$$
\begin{aligned}
& {[A]^{r} \subset K_{10},} \\
& {[A]^{r} \subset K_{11}}
\end{aligned}
$$

holds. If both (61) and (62) are false, then there are sets $X, Y \subset A$ such that

$$
\begin{aligned}
& X=\left\{x_{0}, \cdots, x_{r-1}\right\}_{<}=\left\{x_{0}, \cdots, x_{r-1}\right\}_{<<,} \\
& Y=\left\{y_{0}, \cdots, y_{r-1}\right\}_{<}=\left\{y_{0}, \cdots, y_{r-1}\right\}_{\gg>}
\end{aligned}
$$

Then there is $\sigma<r$ such that $x_{\rho}=y_{\rho}(\rho<\sigma) ; x_{\sigma} \neq y_{\sigma}$. We choose $X$ and $Y$ such that $\sigma$ is as large as possible. We may assume that $x_{\sigma}<y_{\sigma}$. Then, if we suppose that $\sigma+1<r$, we find that $\left\{y_{0}, \cdots, y_{\sigma-1}, x_{\sigma}\right.$, $\left.y_{\sigma}, \cdots, y_{r-2}\right\}_{<}$and therefore, by the maximum property of $\sigma$, $\left\{y_{0}, \cdots, y_{\sigma-1}, x_{\sigma}, y_{\sigma}, \cdots, y_{r-2}\right\}<$. Hence $\left\{y_{0}, \cdots, y_{\sigma-1}, y_{\sigma}, \cdots\right.$, $\left.y_{r-2}\right\}_{<<}$and therefore, since $r-2>0, y_{0} \ll y_{r-2}$ which contradicts (64). Therefore $\sigma+1=r$, and $x_{0}=y_{0} \gg y_{1}=x_{1}$. But this contradicts (63). This shows that at least one of the relations (61), (62) holds. Now (61) implies $\beta=\bar{A}_{<}=\bar{A}_{<<} \leqq \bar{S}_{<<}=\alpha$, and (62) implies $\beta^{*}=\bar{A}_{>}$ $=\bar{A}_{<<} \leqq \bar{S}_{<<}=\alpha$. Both conclusions contradict the hypothesis. We have proved that neither Case 1 nor Case 2 is possible, so that (56) is established. This proves Theorem 35.

Theorem 36. (i) If $a_{\nu}<d(\nu<n)$, then $\sum[\nu<n] a_{\nu} \rightarrow(|n|+, d)^{2}$.

(ii) $a \rightarrow\left(a^{\prime+}, a\right)^{2}$ for $a>1$.

(iii) $a b \rightarrow\left(a^{+}, b^{+}\right)^{2}$ for any $a, b$.

(iv) $\aleph_{n} \rightarrow\left(|n|+, \aleph_{n}\right)^{2}$, if $n=n^{-}>0$.

Proof of (i). Let $\left|A_{\nu}\right|=a_{\nu}(\nu<n) ; S=\sum^{\prime}[\nu<n] A_{\nu}$;

$$
[S]^{2}=K_{0}+{ }^{\prime} K_{1} ; \quad K_{1}=\sum[\nu<n]\left[A_{\nu}\right]^{2} .
$$

Then $[X]^{2} \subset K_{0}$ implies $\left|X A_{\nu}\right| \leqq 1(\nu<n) ;|X| \leqq|n|$, and $[X]^{2} \subset K_{1}$ implies the existence of $\nu<n$ such that $X \subset A_{\nu} ;|X| \leqq\left|A_{\nu}\right|<d$. This proves (i).

Proof of (ii). By definition of $a^{\prime}$, the hypothesis of (i) holds for 
some $a_{v}, n$, with $|n|=a^{\prime} ; d=a=\sum a_{v}$. Hence (ii) follows from (i).

ProOF OF (iii). Let $|n|=a ; a_{\nu}=b(\nu<n)$. Then, by (i),

$$
a b=\sum a_{\nu} \rightarrow\left(a^{+}, b^{+}\right)^{2} .
$$

PROOF OF (iv). Since $\boldsymbol{\aleph}_{n}=\sum[\nu<n] \boldsymbol{\aleph}_{\nu}$, (iv) follows from (i). This proves Theorem 36 .

THEOREM 37. (i) Let $a \geqq \boldsymbol{N}_{0}$, and let $b$ be minimal such that $a^{b}>a$. Let $a<\boldsymbol{\aleph}_{k}^{\prime} \leqq \boldsymbol{\aleph}_{k} \leqq a^{b}$. Then

$$
\boldsymbol{\aleph}_{\omega_{k}} \rightarrow\left(b^{+}, \boldsymbol{\aleph}_{\omega_{k}}\right)^{2} .
$$

$A$ possible value for $\boldsymbol{\aleph}_{k}$ is $a^{+}$.

(ii) $\boldsymbol{\aleph}_{\omega_{m+1}} \rightarrow\left(\boldsymbol{\aleph}_{m+1}, \boldsymbol{\aleph}_{\omega_{m+1}}\right)^{2}$ for all $m$.

(iii) If $\boldsymbol{\aleph}_{0}<\boldsymbol{\aleph}_{k}^{\prime} \leqq \boldsymbol{\aleph}_{k} \leqq 2^{\boldsymbol{\aleph}_{0}}$, then $\boldsymbol{\aleph}_{\omega_{k}} \rightarrow\left(\boldsymbol{\aleph}_{1}, \boldsymbol{\aleph}_{\omega_{k}}\right)^{2}$. A possible value is $k=1$.

Deduction of (ii) from (i). Let $a=\boldsymbol{\aleph}_{m}$, and let $b$ be minimal such that $a^{b}>a$. Then, if $k=m+1$, we have $a<\boldsymbol{\aleph}_{k}^{\prime}=\boldsymbol{\aleph}_{k} \leqq a^{b}$ and therefore, by (i), $\boldsymbol{\aleph}_{\omega_{m+1}} \rightarrow\left(b^{+}, \boldsymbol{\aleph}_{\omega_{m+1}}\right)^{2}$. This implies (ii).

Deduction of (iii) from (i). Put, in (i), $a=b=\boldsymbol{\aleph}_{0}$. Then (iii) follows.

Before proving (i) we establish a lemma. For the sake of further applications later on the lemma is more general than is needed for the present purpose.

Lemma 4. If $s \geqq 2 ; \beta_{0}, \beta_{1}^{*} \ddagger \alpha_{0} ;\left|\alpha_{0}\right|=\left|\alpha_{1}\right|$, then

$$
\alpha_{1} \rightarrow\left(\beta_{0}, \beta_{1}, s+1, s+1, \cdots, s+1\right)_{s !}^{s} \text {. }
$$

Proof. Let $\bar{S}_{<}=\alpha_{1} ; \bar{S}_{<<}=\alpha_{0}$. Then to every set $X \in[S]^{\text {s }}$ there belongs a permutation $\pi(X): \lambda \rightarrow \sigma(\lambda)$ defined by

$$
X=\left\{x_{0}, x_{1}, \cdots, x_{s-1}\right\}_{<}=\left\{x_{\sigma(0)}, x_{\sigma(1)}, \cdots, x_{\sigma(s-1)}\right\}_{<<}
$$

Let $\pi_{\lambda}(\lambda<s !)$ be all permutations of $[0, s]$ and, in particular,

$$
\pi_{0}: \lambda \rightarrow \lambda ; \quad \pi_{1}: \lambda \rightarrow s-1-\lambda \quad(\lambda<s) .
$$

Then $[S]^{s}=\sum[\nu<s !] K_{\nu}$, where $K_{\nu}=\left\{X: X \in[S]^{s} ; \pi(X)=\pi_{\nu}\right\}$. Now suppose that $A \subset S ; \nu<s ! ;[A]^{\circ} \subset K_{\nu}$. We shall deduce a contradiction in each of the three cases that follow and so establish the lemma.

Case 1. $\nu=0 ; \bar{A}_{<}=\beta_{0}$. Then the contradiction $\beta_{0}=\bar{A}_{<<} \leqq \bar{S}_{<<}=\alpha_{0}$ follows.

Case 2. $\nu=1 ; \bar{A}_{<}=\beta_{1}$. Then the contradiction $\beta_{1}^{*}=\bar{A}_{<<\leqq} \bar{S}_{<<}=\alpha_{0}$ follows.

Case 3. $2 \leqq \nu<s ! ; \bar{A}_{<}=s+1$. Let $\pi_{\nu}: \lambda \rightarrow \sigma(\lambda)$. Then $A=\left\{x_{0}\right.$, $\left.x_{1}, \cdots, x_{s}\right\}<$ and therefore, putting $y_{\lambda}=x_{1+\lambda}(\lambda<s)$, we have 


$$
\begin{aligned}
\left\{x_{0}, x_{1}, \cdots, x_{s-1}\right\}_{<} & =\left\{x_{\sigma(0)}, x_{\sigma(1)}, \cdots, x_{\sigma(s-1)}\right\}_{<<,} \\
\left\{x_{1}, x_{2}, \cdots, x_{s}\right\}_{<} & =\left\{y_{0}, y_{1}, \cdots, y_{s-1}\right\}_{<} \\
& =\left\{y_{\sigma(0)}, y_{\sigma(1)}, \cdots, y_{\sigma(s-1)}\right\}_{<<} \\
& =\left\{x_{1+\sigma(0)}, x_{1+\sigma(1)}, \cdots, x_{1+\sigma(s-1)}\right\}<<
\end{aligned}
$$

If $x_{0} \ll x_{1}$, then alternate applications of (66) and (67) lead to $x_{0} \ll x_{1} \ll x_{2} \ll \cdots \ll x_{s}$ and so to the contradiction $\pi_{\nu}=\pi_{0}$, while, similarly, the assumption $x_{0} \gg x_{1}$ leads to the contradiction $\pi_{\nu}=\pi_{1}$. This proves the lemma.

Proof of Theorem 37, (i). Let $a=\boldsymbol{\aleph}_{m} ; b=\boldsymbol{\aleph}_{l}$, and let $F$ be the set of all mappings $\lambda \rightarrow h(\lambda)$ of $\left[0, \omega_{l}\right]$ into $\left[0, \omega_{m}\right]$. We order $F$ by putting, for $h_{0}, h_{1} \in F, h_{0} \ll h_{1}$ if, and only if, there is $\lambda_{0}<\omega_{l}$ such that

$$
h_{0}(\lambda)=h_{1}(\lambda) \text { for } \lambda<\lambda_{0} ; \quad h_{0}\left(\lambda_{0}\right)<h_{1}\left(\lambda_{0}\right) .
$$

Then $|F|=a^{b}$, and we have, by ${ }^{9}$ Lemma 2 of [6], if $a=\boldsymbol{N}_{m} ; b=\boldsymbol{N}_{l}$,

$$
\omega_{m+1}, \omega_{l+1}^{*} \$ \bar{F}_{<<}=\bar{F} \text {, say. }
$$

We can choose a set $X \in[F]{ }_{k}$. Let $x \rightarrow f(x)$ be a one-one mapping of $X$ on $\left[0, \omega_{k}\right]$, and $S=\left\{(x, \nu): x \in X ; \nu<\omega_{f(x)}\right\}$. We order $S$ alphabetically, by means of a relation $u<v$, and put $\bar{S}_{<}=\bar{S}=\phi$. Then

$$
|S|=\sum[x \in X] \boldsymbol{\aleph}_{f(x)} \leqq \sum\left[\lambda<\omega_{k}\right] \boldsymbol{\aleph}_{\omega_{k}}=\boldsymbol{\aleph}_{\omega_{k}} \text {. }
$$

On the other hand, if $d<\boldsymbol{\aleph}_{\omega_{k}}$, then $d<\boldsymbol{\aleph}_{n}$, for some $n<\omega_{k}$, and there is $x_{0} \in X$ such that $f\left(x_{0}\right)>n$. Then $|S| \geqq \boldsymbol{\aleph}_{f\left(x_{0}\right)}>d$. Hence

$$
|\phi|=|S|=\boldsymbol{N}_{\omega_{k}} \text {. }
$$

1. Let $S_{1} \subset S$, and suppose that $\bar{S}_{1}$ is an ordinal. Put $X_{1}=\sum\left[\nu<\omega_{k}\right]$ - $\left\{x:(x, \nu) \in S_{1}\right\}$. Then $\bar{X}_{1}$ is an ordinal, and $\bar{X}_{1} \leqq \bar{F}$. Hence, by (68), $\bar{X}_{1}<\omega_{m+1} ;\left|X_{1}\right| \leqq \boldsymbol{N}_{m}=a<\boldsymbol{N}_{k}^{\prime}$.

$$
\begin{gathered}
\sum\left[x \in X_{1}\right]|f(x)|<\boldsymbol{\aleph}_{k} ; \quad \nu_{1}=\sum\left[x \in X_{1}\right] f(x)<\omega_{k} ; \\
\left|S_{1}\right|=\sum\left[x \in X_{1}\right]\left|\left\{\nu:(x, \nu) \in S_{1}\right\}\right| \leqq \sum\left[x \in X_{1}\right] \boldsymbol{\aleph}_{f(x)} \\
\leqq \boldsymbol{\aleph}_{\nu_{1}}\left|X_{1}\right| \leqq \boldsymbol{\aleph}_{\nu_{1}} \boldsymbol{\aleph}_{m}<\boldsymbol{\aleph}_{\omega_{k}} ; \\
\omega_{\omega_{k}} \leqq \phi .
\end{gathered}
$$

2. Let $S_{2} \subset S$, and suppose that $\left(\bar{S}_{2}\right)^{*}$ is an ordinal. Put $X_{2}=\sum\left[\nu<\omega_{k}\right]\left\{x:(x, \nu) \in S_{2}\right\}$. Then $\left(\bar{X}_{2}\right)^{*}$ is an ordinal, and $\left(\bar{X}_{2}\right)^{*}$ $\leqq \bar{F}$. Hence, by (68), $\left(\bar{X}_{2}\right)^{*}<\omega_{l+1} ;\left|X_{2}\right| \leqq \aleph_{l}$. Put, for $x \in X_{2}, N(x)$ $=\left\{\nu:(x, \nu) \in S_{2}\right\}$. Then $\overline{N(x)}$ is an ordinal. On the other hand,

9 The authors are indebted to G. Kurepa for pointing out that the result of this lemma had already been obtained by F. Hausdorff, [9, Satz 14]. 
$(\overline{N(x)}) *$ is an ordinal, since $(\overline{N(x)})$ $\leqq\left(\bar{S}_{2}\right) *$. Hence $\overline{N(x)}<\omega_{0}$;

$$
\begin{gathered}
\left|S_{2}\right|=\sum\left[x \in X_{2}\right]|N(x)| \leqq \aleph_{0}\left|X_{2}\right| \leqq \aleph_{l} ; \quad\left(\bar{S}_{2}\right)^{*}<\omega_{l+1} ; \\
\omega_{l+1}^{*} \leqq \phi .
\end{gathered}
$$

3. We now apply Lemma 4 to the case

$$
s=2 ; \quad \alpha_{0}=\phi ; \quad \alpha_{1}=\omega_{\omega_{k}} ; \quad \beta_{0}=\omega_{\omega_{k}} ; \quad \beta_{1}=\omega_{l+1} .
$$

Its hypotheses are satisfied, by (70), (71), (69). We obtain $\omega_{\omega_{k}}$ $\rightarrow\left(\omega_{\omega_{k}}, \omega_{l+1}\right)^{2}$. This implies (65), by Theorem 14, and completes the proof of Theorem 37.

REMARK. If $a \geqq \boldsymbol{\aleph}_{0}$ and $\boldsymbol{\aleph}_{k}=a^{+}$, then (i) of Theorem 37 yields a stronger result then (ii) of Theorem 36 . For, first of all, we note that the hypothesis of (i) of Theorem 37 holds, since $a<a^{+}=a^{+^{\prime}}=\boldsymbol{\aleph}_{k}^{\prime}$ $=\boldsymbol{\aleph}_{k} \leqq a^{b}$. Hence the latter theorem gives

$$
\boldsymbol{\aleph}_{\omega_{k}} \rightarrow\left(b^{+}, \boldsymbol{\aleph}_{\omega_{k}}\right)^{2} \text {. }
$$

On the other hand, (ii) of Theorem 36 gives

$$
\boldsymbol{\aleph}_{\omega_{k}} \rightarrow\left(\boldsymbol{\aleph}_{\omega_{k}}^{\prime+}, \boldsymbol{\aleph}_{\omega_{k}}\right)^{2}
$$

It is known that, for any $m$,

$$
\boldsymbol{\aleph}_{\omega_{m}}^{\prime}=\boldsymbol{\aleph}_{m}^{\prime} \text {. }
$$

Hence $\boldsymbol{\aleph}_{\omega_{k}}^{\prime+}>\boldsymbol{\aleph}_{\omega_{k}}^{\prime}=\boldsymbol{\aleph}_{k}^{\prime}=a^{+\prime}=a^{+} \geqq b^{+}$, and (72) is stronger than (73).

Since we were not able to find a reference for (74) we give, for the sake of completeness, a proof now.

Case 1. Let $\boldsymbol{\aleph}_{\omega_{m}}^{\prime}=\boldsymbol{\aleph}_{n}<\boldsymbol{\aleph}_{m}^{\prime}$. Then $\boldsymbol{\aleph}_{\omega_{m}}=\sum\left[\nu<\omega_{n}\right] \boldsymbol{\aleph}_{\lambda_{\nu}}$, for some $\lambda_{\nu}<\omega_{m}$. Put $\lambda=\sum\left[\nu<\omega_{n}\right] \lambda_{\nu}$. Then, since $\left|\omega_{n}\right|<\boldsymbol{\aleph}_{m}^{\prime} ;\left|\lambda_{\nu}\right|<\boldsymbol{\aleph}_{m}$ we conclude that

$$
\begin{aligned}
& |\lambda|=\sum\left[\nu<\omega_{n}\right]\left|\lambda_{\nu}\right|<\boldsymbol{\aleph}_{m} ; \quad \boldsymbol{\aleph}_{n}<\boldsymbol{\aleph}_{m}^{\prime} \leqq \boldsymbol{\aleph}_{m} \leqq \boldsymbol{\aleph}_{\omega_{m}} ; \\
& \boldsymbol{\aleph}_{\omega_{m}} \leqq \sum\left[\nu<\omega_{n}\right] \boldsymbol{\aleph}_{\lambda}=\boldsymbol{\aleph}_{\lambda} \boldsymbol{\aleph}_{n}<\boldsymbol{\aleph}_{\omega_{m}}
\end{aligned}
$$

which is the desired contradiction.

Case 2. Let $\boldsymbol{\aleph}_{\omega_{m}}^{\prime}>\boldsymbol{\aleph}_{n}=\boldsymbol{\aleph}_{m}^{\prime}$. Then $m>0$, and $\boldsymbol{\aleph}_{m}=\sum\left[\nu<\omega_{n}\right] \boldsymbol{\aleph}_{\lambda_{\nu}}$ for some $\lambda_{\nu}<m$. Then $\sum\left[\nu<\omega_{n}\right] \boldsymbol{\aleph}_{\omega_{\lambda_{\nu}}}=\boldsymbol{\aleph}_{l}$ for some $l<\omega_{m} ; \boldsymbol{\aleph}_{\lambda_{\nu}}=\left|\omega_{\lambda_{\nu}}\right|$ $\leqq|l| ; \quad \boldsymbol{\aleph}_{m}=\sum\left[\nu<\omega_{n}\right] \boldsymbol{\aleph}_{\lambda_{\nu}} \leqq|l| \boldsymbol{\aleph}_{n} ; \quad m \leqq n ; \quad \boldsymbol{\aleph}_{\omega_{m}}=\sum\left[\mu<\omega_{m}\right] \boldsymbol{\aleph}_{\mu} ;$ $\boldsymbol{\aleph}_{\omega_{m}}^{\prime} \leqq\left|\omega_{m}\right| \leqq \boldsymbol{\aleph}_{n}$ which, again, is a contradiction. This proves (74).

Theorem 38. If $\boldsymbol{\aleph}_{m} \rightarrow\left(\left|\beta_{0}\right|,\left|\beta_{1}\right|, \cdots\right)_{\boldsymbol{k}}^{r}$, then

$$
\omega_{m+1} \rightarrow\left(\beta_{0}+1, \beta_{1}+1, \cdots\right)_{k}^{r+1} \text {. }
$$


We give some applications of this theorem.

(i) If $|\beta|=|\gamma|=\aleph_{m+1}$, then

$$
\omega_{m+2} \rightarrow(\beta+1, \gamma+1)^{3} \text {. }
$$

For, let $a=\boldsymbol{\aleph}_{m}$, and let $b$ be minimal such that $a^{b}>a$. Then, by Theorem 7, $a^{b} \rightarrow\left(a^{+}, b^{+}\right)^{2}$ and therefore $\aleph_{m+1} \rightarrow(|\beta|,|\gamma|)^{2}$. Now (76) follows from Theorem 38 .

(ii) If $\boldsymbol{\aleph}_{n}^{\prime}=\boldsymbol{\aleph}_{m} ;|\beta|=\boldsymbol{\aleph}_{m+1} ;|\gamma|=\boldsymbol{\aleph}_{\omega_{n}}$, then

$$
\omega_{\omega_{n}+1} \rightarrow(\beta+1, \gamma+1)^{3} \text {. }
$$

In order to prove (77), we apply Theorem 36 (ii) to $a=\boldsymbol{N}_{\omega_{n}}$. We note that, by (74), $a^{\prime}=\boldsymbol{\aleph}_{n}^{\prime}=\boldsymbol{\aleph}_{m} ; a^{\prime+}=\boldsymbol{\aleph}_{m+1}$. Hence, by Theorem 36 , $\aleph_{\omega_{n}} \rightarrow(|\beta|,|\gamma|)^{2}$, and (77) follows from Theorem 38 .

(iii) If $|\beta|=\aleph_{k+1} ;|\gamma|=\aleph_{\omega_{k+1}}$, then

$$
\omega_{\omega_{k+1}+1}+(\beta+1, \gamma+1)^{3} \text {. }
$$

This follows immediately from Theorem 37 (ii) and an application of Theorem 38 .

We note that on putting $n=k+1$ in (ii) above one obtains a result which is weaker than (78). For, (ii) becomes: if $\boldsymbol{N}_{k+1}=\boldsymbol{N}_{m} ;|\beta|=\boldsymbol{N}_{k+2}$; $|\gamma|=\mathbf{N}_{\omega_{k+1}}$, then (78) holds.

The proof of Theorem 38 depends on a lemma.

Lemma 5. Let $\alpha$ be an ordinal. Suppose that $\beta_{\nu}(\nu<k)$ and $r$ are such that, whenever $\beta<\alpha$, then $\beta \rightarrow\left(\beta_{0}, \beta_{1}, \cdots\right)_{k}^{r}$. Then

$$
\alpha \rightarrow\left(\beta_{0}+1, \beta_{1}+1, \cdots\right)_{k}^{r+1} \text {. }
$$

Proof. Let $\bar{S}=\alpha ; x \in S$. Then $\overline{L(x)}<\alpha$, and hence, by hypothesis, there is a partition $[L(x)]^{r}=\sum[\nu<k] K_{\nu}(x)$ such that, whenever $X \subset L(x) ; \quad[X] r \subset K_{\nu}(x)$, then $\bar{X}<\beta_{\nu}$. Put $K_{\nu}=\{A+\{x\}: x \in S$; $\left.A \in K_{\nu}(x)\right\}(\nu<k)$. Then $[S]^{r+1}=\sum[\nu<k] K_{\nu}$. If we now assume that

$$
S^{\prime} \subset S ; \quad\left[S^{\prime}\right]^{r+1} \subset K_{\nu} ; \quad \bar{S}^{\prime}=\beta_{\nu}+1,
$$

then $S^{\prime}=S^{\prime \prime}+\left\{x^{\prime}\right\} ; S^{\prime \prime} \subset L\left(x^{\prime}\right) ; \bar{S}^{\prime \prime}=\beta_{\nu} ;\left[S^{\prime \prime}\right] r \subset K_{\nu}\left(x^{\prime}\right)$ which contradicts the definition of $K_{\nu}\left(x^{\prime}\right)$. Hence (80) is impossible, and (79) follows.

ProOF of TheOREM 38. If $\beta<\omega_{m+1}$, then $|\beta| \leqq \aleph_{m},|\beta| \rightarrow\left(\left|\beta_{0}\right|\right.$, $\left.\left|\beta_{1}\right|, \cdots\right)_{k}^{r}$. By Theorem 13, this implies $\beta \rightarrow\left(\beta_{0}, \beta_{1}, \cdots\right)_{k}^{r}$. Now (75) follows from Lemma 5.

THEOREM 39. (i) If

$$
l \rightarrow\left(\alpha_{0}, \alpha_{1}, \cdots\right)_{k}^{r}
$$




$$
|m|>\sum[\lambda<l]|k|^{|\lambda|^{r}}
$$

then

$$
m \rightarrow\left(\alpha_{0}+1, \alpha_{1}+1, \cdots\right)_{k}^{r+1} \text {. }
$$

(ii) If $r>0 ; \omega_{n} \rightarrow\left(\alpha_{0}, \alpha_{1}, \cdots\right)_{k}^{r}$, and

$$
2^{\aleph_{\nu}} \leqq \aleph_{n}
$$

$$
\text { for } \nu<n \text {, }
$$

then

$$
\omega_{n+1} \rightarrow\left(\alpha_{0}+1, \alpha_{1}+1, \cdots\right)_{k}^{r+1} \text {. }
$$

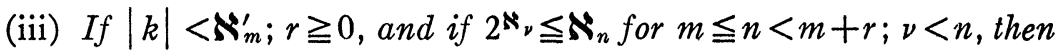

$$
\begin{aligned}
& \omega_{m+r} \rightarrow\left(\omega_{m}+r\right)_{k}^{r+1}, \\
& \omega_{m+r} \rightarrow\left(\omega_{m}+r\right)_{k}^{r} .
\end{aligned}
$$

Deduction of (ii) from (i). Let the hypothesis of (ii) hold. If $\left|\alpha_{\nu}\right|<r$ for some $\nu<k$, then the assertion is trivial, by Theorem 22. Hence we may assume that $\left|\alpha_{\nu}\right| \geqq r(\nu<k)$. Next, suppose that $\left|\alpha_{\nu}\right|=r$ for some $\nu<k$. Then, by Theorem 17, we may apply a suitable permutation to the system $\alpha_{0}, \alpha_{1}, \cdots$ so that for the new system, again denoted by $\alpha_{0}, \alpha_{1}, \cdots, \alpha_{\nu}, \cdots(\nu<k)$, we have

$$
\alpha_{\nu}=r \quad\left(\nu<k_{0}\right) ; \quad\left|\alpha_{\nu}\right|>r \quad\left(k_{0} \leqq \nu<k\right) .
$$

Here $k_{0}$ is some ordinal, $0<k_{0} \leqq k$, and we can write $k=k_{0}+k_{1}$. Then, by Theorem 20 , (ii), the hypothesis implies $\omega_{n} \rightarrow\left(\alpha_{k_{0}}, \alpha_{k_{0+1}}, \cdots\right)_{k_{1}}^{r}$, and the assertion is implied by

$$
\omega_{n+1} \rightarrow\left(\alpha_{k_{0}}+1, \alpha_{k_{0}+1}+1, \cdots\right)_{k_{1}}^{r+1} \text {. }
$$

This shows that we may assume, without loss of generality, that $\left|\alpha_{\nu}\right|>r$ for $\nu<k$. Let us, now, suppose that $|k| \geqq \boldsymbol{\aleph}_{n}$. Then, if $A=\left[0, \omega_{n}\right]$, we can write $[A] r=\sum[\nu<k] K_{\nu}$, where $\left|K_{\nu}\right| \leqq 1$ for $\nu<k$. Since $\bar{A} \rightarrow\left(\alpha_{0}, \cdots\right)_{k}^{r}$, there are $X \subset A, \nu<k$ such that $\bar{X}=\alpha_{\nu}$; $[X]^{r} \subset K_{\nu}$. Then $|X|=\left|\alpha_{\nu}\right|>r ; 1<\left|[X]^{r}\right| \leqq\left|K_{\nu}\right|$ which is a contradiction. This proves that $|k|<\boldsymbol{N}_{n}$.

Put

$$
\sum\left[\lambda<\omega_{n}\right]|k|^{|\lambda|^{r}}=a .
$$

It suffices to show that $\left|\omega_{n+1}\right|>a$.

If $n=0$, then $a<\aleph_{0}$. If $n>0$, then

$$
a \leqq \sum\left[\lambda<\omega_{n}\right] 2^{|k||\lambda|^{r}} \leqq \sum\left[\lambda<\omega_{n}\right] 2^{\wedge \nu_{\lambda}},
$$

for some $\nu_{\lambda}<n$. Hence, by (84), $a \leqq \sum\left[\lambda<\omega_{n}\right] \boldsymbol{\aleph}_{n}=\boldsymbol{\aleph}_{n}$. 
Deduction of (iii) from (ii). By definition of $\boldsymbol{\aleph}_{m}^{\prime}, \omega_{m} \rightarrow\left(\omega_{m}\right)_{k}^{1}$. Hence, by $r$ applications of (ii), (85) follows. Now (86) follows from Theorem 15.

Proof of (i). Let $\bar{S}=m ;[S]^{r+1}=\sum^{\prime}[\nu<k] K_{\nu}$. We choose $n$ such that $|n|>|m|$. Throughout this proof the letters $\kappa, \lambda, \rho, \sigma$ denote ordinals less than $n$, and $x, y$ and $z$ elements of $S$. The relation

$$
\left\{x_{0}, \cdots, x_{r}\right\} \equiv\left\{y_{0}, \cdots, y_{r}\right\}
$$

expresses, by definition, the fact that, for some $\nu<k$,

$$
\left\{x_{0}, \cdots, x_{r}\right\},\left\{y_{0}, \cdots, y_{r}\right\} \in K_{r} .
$$

We define $f_{\kappa}(x) \in S$ as follows. Let $x$ be fixed, and suppose that, for some fixed $\lambda$, the elements $f_{\kappa}=f_{\kappa}(x)$ have already been defined for all $\kappa<\lambda$. Then we put $f_{\lambda}(x)=x$, if $f_{\kappa}=x$ for some $\kappa<\lambda$. If, on the other hand, $f_{\kappa} \neq x$ for $\kappa<\lambda$, then we define $f_{\lambda}$ to be the first element $y$ of $S-\left\{f_{\kappa}: \kappa<\lambda\right\}$ such that

$$
\left\{f_{\kappa_{0}}, \cdots, f_{\kappa_{r-1}}, y\right\} \equiv\left\{f_{\kappa_{0}}, \cdots, f_{\kappa_{r-1}}, x\right\} \text { for } \kappa_{0}<\cdots<\kappa_{r-1}<\lambda .
$$

This defines $f_{\kappa}$ for all $\kappa$. We now prove that

$$
f_{\lambda}<f_{\mu}
$$

if

$$
\lambda<\mu ; \quad f_{\lambda} \neq x .
$$

First of all, (87) holds for $y=x$. Hence, by (89) and the definition of $f_{\lambda}$, we have $f_{\lambda}<x$. This proves (88) in the case when $f_{\mu}=x$. Now suppose that $f_{\mu} \neq x$. Then (87) holds for $y=f_{\mu}$ and, again, (88) follows. By (88) and $|n|>|m|$, there is $\rho(x)$ such that

$$
f_{\kappa}(x)<f_{\lambda}(x)=x, \quad \text { if } \kappa<\rho(x) \leqq \lambda .
$$

Let, for $\kappa_{0}<\cdots<\kappa_{r-1}<\rho(x)$,

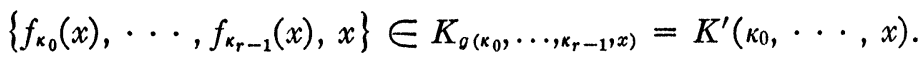

We now show that if $x$ and $z$ are such that

$$
\begin{aligned}
& \quad \rho(x)=\rho(z), \\
& K^{\prime}\left(\kappa_{0}, \cdots, \kappa_{r-1}, x\right) \\
& =K^{\prime}\left(\kappa_{0}, \cdots, \kappa_{r-1}, z\right) \text { for } \kappa_{0}<\cdots<\kappa_{r-1}<\rho(x),
\end{aligned}
$$

then $x=z$. Let $\lambda \leqq \rho(x)$, and suppose that

$$
f_{k}(x)=f_{k}(z) \quad \text { for } \kappa<\lambda .
$$


Then $f_{\lambda}(x)$ is the first element $y$ of $S-\left\{f_{\kappa}(x): \kappa<\lambda\right\}$ such that (87) holds, i.e.

$$
\left\{f_{\kappa_{0}}(x), \cdots, f_{\kappa_{r-1}}(x), y\right\} \in K^{\prime}\left(\kappa_{0}, \cdots, \kappa_{r-1}, x\right) \text { for } \kappa_{0}<\cdots<\kappa_{r-1}<\lambda .
$$

Now, $f_{\lambda}(z)$ is defined by the same property, with $z$ in place of $x$, and (90) and (91) show that $f_{\lambda}(x)=f_{\lambda}(z)$. We have thus proved, by induction, that $f_{\kappa}(x)=f_{\kappa}(z)$ for all $\kappa \leqq \rho(x)$. In particular, by $(90)$,

$$
x=f_{\rho(x)}(x)=f_{\rho(z)}(z)=z \text {. }
$$

We next prove that $\rho\left(x_{0}\right) \geqq l$ for at least one $x_{0}$. Let us suppose, on the contrary, that $\rho(x)<l$ for all $x$. Let $\sigma<l$. Then the cardinal $a(\sigma)$ of the set $\{x: \rho(x)=\sigma\}$ is at most equal to the cardinal of the set of all functions $h\left(\kappa_{0}, \cdots, \kappa_{r-1}\right)<k$, defined for $\kappa_{0}<\cdots<\kappa_{r-1}<\sigma$. Hence

$$
\begin{aligned}
a(\sigma) & \leqq|k||\sigma|^{r} ; \quad|m|=|S|=\left|\sum[\sigma<l]\{x: \rho(x)=\sigma\}\right| \\
& \leqq \sum[\sigma<l]|k|^{\left.|| \sigma\right|^{r}},
\end{aligned}
$$

which contradicts $(82)$. This proves that $\rho\left(x_{0}\right) \geqq l$ for some suitable $x_{0}$. Put $S_{0}=\left\{f_{x}\left(x_{0}\right): \kappa<l\right\}$. Then $\bar{S}_{0}=l ;\left[S_{0}\right] r=\sum[\nu<k] K_{\nu}^{\prime \prime}$, where $K_{\nu}^{\prime \prime}=\left\{A: A \in\left[S_{0}\right] r ; A+\left\{x_{0}\right\} \in K_{\nu}\right\} \quad(\nu<k)$. By (81), there are $S_{1} \subset S_{0} ; \nu<k$ such that $\bar{S}_{1}=\alpha_{\nu} ;\left[S_{1}\right]^{r} \subset K_{\nu}^{\prime \prime}$. Then the set $S_{2}=S_{1}+\left\{x_{0}\right\}$ satisfies $\bar{S}_{2}=\alpha_{\nu}+1 ;\left[S_{2}\right]^{r+1} \subset K_{\nu}$, and (83) follows. This completes the proof of Theorem 39 .

Corollary of Theorem 39. Given any $r$ and any ordinals $k, \beta_{\nu}$, there always exists an ordinal $\alpha$ such that

$$
\alpha \rightarrow\left(\beta_{0}, \beta_{1}, \cdots\right)_{k}^{r} \text {. }
$$

For, if $r \leqq 1$, then any $\alpha$ can be taken such that $|\alpha|>\sum[\nu<k]\left|\beta_{\nu}\right|$, and the result for $r=2,3, \cdots$ is obtained by applications of Theorem 39 . The relation (5) shows that the last proposition becomes false if $\alpha$ and $\beta_{\nu}$, instead of being ordinals, are allowed to be any order types. Later on (Theorem 45) the corollary will be extended, for $r=1$, to the case of arbitrary types $\beta_{v}$.

We mention, without proof, the following further applications of Theorem 39 (i).

(a) $\left(2^{a}\right)^{+} \rightarrow(a)_{k}^{2}$ if $|k|<a^{\prime}$.

(b) $a^{+} \rightarrow(a)_{k}^{2} ; \omega_{n+1} \rightarrow\left(\omega_{n}+1\right)_{k}^{2}$, if $a=\boldsymbol{\aleph}_{n} ;|k|<a^{\prime}$, and $2^{b} \leqq a$ for all $b<a$.

(c) $\left(k^{\alpha_{0}+\cdots+\alpha_{k-1}-2 k+1}+k-2\right)(k-1)^{-1} \rightarrow\left(\alpha_{0}, \cdots, \alpha_{k-1}\right)_{k}^{2}$, if $2 \leqq k$, $\alpha_{0}, \cdots, \alpha_{k-1}<\omega_{0}$

If in Theorem 39 we take as $k, l, \alpha_{\nu}$ finite numbers we obtain a result which implies Theorem 1 of [5]. Denote, in this special case, by 


$$
\rho_{k}\left(r ; \alpha_{0}, \alpha_{1}, \cdots, \alpha_{k-1}\right)
$$

the least number $n$ such that

$$
n \rightarrow\left(\alpha_{0}, \cdots, \alpha_{k-1}\right)_{k}^{r} \text {. }
$$

Without loss of generality, we restrict ourselves to the case $k \geqq 2$; $0<r \leqq \alpha_{\nu}$. In [5, Theorem 1], an explicit upper estimate was given for the number $\rho_{k}(r ; \alpha, \alpha, \cdots, \alpha)$, which, in that paper, was denoted by $R(k, r, \alpha)$.

Clearly, $\rho_{k}\left(1 ; \alpha_{0}, \cdots, \alpha_{k-1}\right)=1+\alpha_{0}+\cdots+\alpha_{k+1}-k$. By Theorem 39,

$$
\begin{aligned}
\rho_{k}\left(r+1 ; \alpha_{0}+1, \alpha_{1}+1, \cdots, \alpha_{k-1}+1\right) \\
\leqq 1+\sum\left[\lambda<\rho_{k}\left(r ; \alpha_{0}, \cdots, \alpha_{k-1}\right)\right] k^{k^{r}} .
\end{aligned}
$$

It is easily proved that, for $l<\omega_{0}$,

$$
1+\sum[\lambda<l] k^{\lambda^{r}} \leqq k^{l^{r}}
$$

For, (94) holds for $l=0$, and if $0<m<\omega_{0}$, and (94) holds for $l=m-1$, then

$$
1+\sum[\lambda<m] k^{\lambda^{r}} \leqq k^{(m-1)^{r}}+k^{(m-1)^{r}} \leqq k^{1+(m-1)^{r}} \leqq k^{m^{r}},
$$

so that (94) holds for $l=m$. We have thus proved the following recurrence relation.

THEOREM 40. If $2 \leqq k<\omega_{0} ; 0<r \leqq \alpha_{\nu}<\omega_{0}(\nu<k)$, and if $\rho_{k}$ is defined as above, then

$$
\rho_{k}\left(r+1 ; \quad \alpha_{0}+1, \cdots, \alpha_{k-1}+1\right) \leqq k^{\rho_{k}\left(r ; \alpha_{0}, \cdots, \alpha_{k-1}\right)^{r} .}
$$

In particular, we have, using the notation of $[5]$,

$$
R(k, r+1, \alpha+1) \leqq k^{R(k, r, \alpha)^{r}}(k \geqq 2 ; 0<r \leqq \alpha) .
$$

This is precisely the recurrence relation established in [5], from which the explicit estimate is deduced at once. This is no coincidence, as the method of proof of the present Theorem 39 is related to that used for proving Theorem 1 of [5].

Theorem 39 implies Theorem 4 (i), i.e.

$$
\left(2 \aleph_{\eta}\right)^{+} \rightarrow\left(\aleph_{n+1}\right)_{\omega_{n}}^{2} \text {. }
$$

For, clearly, $\boldsymbol{\aleph}_{n+1} \rightarrow\left(\boldsymbol{\aleph}_{n+1}\right)_{\omega_{n}}^{1}$, and therefore $\omega_{n+1} \rightarrow\left(\omega_{n+1}\right)_{\omega_{n}}^{1}$. Also,

$$
\sum\left[\lambda<\omega_{n+1}\right]\left|\omega_{n}\right|^{|\lambda|} \leqq \aleph_{n}^{\aleph_{n}} \aleph_{n+1}=2^{\aleph_{n}}=\aleph_{m_{0}},
$$

say. Hence, by Theorem 39 (i), $\omega_{m_{0+1}} \rightarrow\left(\omega_{n+1}+1\right)_{\omega_{n}}^{2}$, and (95) follows. 
THEOREM 41. If $r \geqq 3$, then, for all $n$,

$$
\omega_{n+1} \rightarrow\left(\omega_{n}+2, \omega_{0}+1, r+1, r+1, \cdots, r+1\right)_{(r-1) !}^{r} .
$$

As an application, consider the case $r=3 ; n=0$ :

$$
\omega_{1} \rightarrow\left(\omega_{0}+2, \omega_{0}+1\right)^{3} \text {. }
$$

This should be compared with:

$$
\omega_{1} \rightarrow\left(\omega_{0}+1\right)_{k}^{r} \quad\left(k<\omega_{0} ; r \geqq 0\right)
$$

which follows from Theorem 39 (ii) and Theorem 1.

Proof of Theorem 41. Let $\omega_{n} \leqq \beta<\omega_{n+1}$. We apply Lemma 4 to

$$
s=r-1 ; \quad \alpha_{0}=\omega_{n} ; \quad \alpha_{1}=\beta ; \quad \beta_{0}=\omega_{n}+1 ; \quad \beta_{1}=\omega_{0}
$$

and obtain

$$
\beta \mapsto\left(\omega_{n}+1, \omega_{0}, r, \cdots, r\right)_{(r-1) !}^{r-1} .
$$

This holds, a fortiori, if $\beta<\omega_{n}$. Now Lemma 5 proves (96).

A type $\beta$ is called indecomposable if the equation $\beta=\gamma+\delta$ implies that either $\gamma \geqq \beta$ or $\delta \geqq \beta$. It is known ${ }^{10}$ that the indecomposable ordinals are those of the form $\omega_{0}^{\lambda}$. The types $\eta$ and $\lambda$ are indecomposable. The next theorem asserts that in Lemma 4 the $s !-2$ classes corresponding to the entries $s+1$ in the partition relation may be suppressed in the special case when both $\beta_{0}$ and $\beta_{1}$ are indecomposable, at the cost, however, of raising the remaining entries slightly.

Theorem 42. Let $s \geqq 3 ;\left|\alpha_{0}\right|=\left|\alpha_{1}\right| ; \beta_{0}, \beta_{1}^{*} \ddagger \alpha_{0}$, and suppose that $\beta_{0}$ and $\beta_{1}$ are indecomposable. Then

$$
(s-3)+\alpha_{1} \rightarrow\left((s-3)+\beta_{0},(s-3)+\beta_{1}\right)^{s} .
$$

Proof. Case 1. $s=3$. Consider a set $S$ with two orders such that $\bar{S}_{<}=\alpha_{1} ; \bar{S}_{<<}=\alpha_{0}$. Then $[S]^{3}=K_{0}+^{\prime} K_{1}$, where $K_{0}$ is the set of all sets

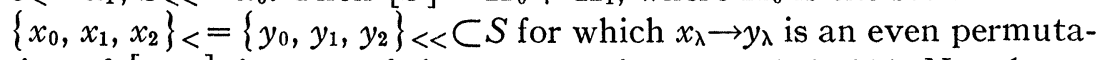
tion of $[0,3]$, i.e. one of the permutations $012,120,201$. Now let us assume that

$$
\alpha_{1} \rightarrow\left(\beta_{0}, \beta_{1}\right)^{3} .
$$

It suffices to deduce a contradiction in each of the two cases that follow.

Case 1.1. There is $A \subset S$ such that $\bar{A}_{<}=\beta_{0} ;[A]^{3} \subset K_{0}$. Let $x, y, z$ denote elements of $A$. Then $\{x, y, z\}_{<}=\left\{x_{1}, y_{1}, z_{1}\right\}_{<<\text {implies that }}$ $x_{1}, y_{1}, z_{1}$ is a cyclic permutation of $x, y, z$. Put $B=\{x: y \ll x$, whenever

$10[13,8875-78]$. 
$y<x\} ; C=A-B$. We shall prove three propositions about the two orders of $A$ showing their effects on the partition $A=B+C$.

1. Let $x<y \in B ; x \in C$. Then there is $z$ such that $z<x \ll z ;\{z, x, y\}<$ $=\{x, y, z\}<<; z<y \ll z ; y \in C$ which is false. Hence $x<y \in B$ implies $x \in B$, as well as $x \ll y$. Therefore $x>y \in C$ implies $x \in C$.

2. Let $x \in B ; y \in C ; x \ll y$. Then $x<y$. There is $z$ such that $z<y \ll z$. Then $\{x, y, z\}_{<<}=\{z, x, y\}<; z<x \ll z ; x \in C$ which is false. Hence $x \in B ; y \in C$ implies $y \ll x$.

3. Let $x, y \in C ; x<y \ll x$. Then there is $z$ such that $z<x \ll z$; $\{z, x, y\}_{<}=\{y, x, z\}_{<<}$which contradicts the definition of $K_{0}$. Hence $x, y \in C ; x<y$ implies $x \ll y$.

The results of 1,2 and 3 show that, if we put $\bar{B}_{<}=\gamma_{0} ; \bar{C}_{<}=\gamma_{1}$, then $\beta_{0}=\bar{A}_{<}=\gamma_{0}+\gamma_{1} ; \bar{A}_{<<}=\gamma_{1}+\gamma_{0}$. Since $\beta_{0}$ is indecomposable, it follows that $\gamma_{\nu} \geqq \beta_{0}$ for some $\nu<2$. Then

$$
\beta_{0} \leqq \gamma_{\nu} \leqq \bar{A}_{<<} \leqq \bar{S}_{<<}=\alpha_{0}
$$

which is a contradiction.

Case 1.2. There is $A \subset S$ such that $\bar{A}_{<}=\beta_{1} ;[A]^{3} \subset K_{1}$. Then

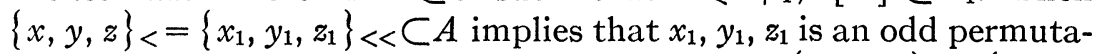
tion of $x, y, z$. This is equivalent to saying that $\{x, y, z\}<=\left\{x_{2}, y_{2}\right.$, $\left.z_{2}\right\}_{\gg>} \subset A$ implies that $x_{2}, y_{2}, z_{2}$ is an even permutation of $x, y, z$. Hence the result of Case 1.1 holds if $\beta_{0}$ is replaced by $\beta_{1}$, and "«" by " $\gg$ ". We note that $\beta_{1}^{*}$ is indecomposable. Hence, in place of $(100)$ we have

$$
\beta_{1} \leqq \bar{A}_{>>} \leqq \bar{S}_{>>}=\alpha_{0}^{*}
$$

which is a contradiction. This shows that the assumption (99) was false, i.e. that (98) holds.

Case 2. $s>3$. Then, by the result of Case 1, we have $\alpha_{1} \rightarrow\left(\beta_{0}, \beta_{1}\right)^{3}$. By Theorem 15, this implies (98). This completes the proof of Theorem 42.

REMARK. If, in particular, $\beta_{0}$ and $\beta_{1}$ are ordinals, not zero, then $(s-3)+\beta_{\nu}=\beta_{\nu}$, so that (98) can be replaced by

$$
(s-3)+\alpha_{1} \rightarrow\left(\beta_{0}, \beta_{1}\right)^{s} .
$$

We may also mention here the following corollary of two of our lemmas, in which $\lambda$ is the type of the continuum.

$$
\text { If } 2 \aleph_{0}=\aleph_{n}, \quad \text { then } \omega_{n+1} \rightarrow\left(\omega_{1}+1, \omega_{1}+1\right)^{3} .
$$

Proof. Let $\omega_{n} \leqq \alpha_{1}<\omega_{n+1}$. Then, by Lemma 4 , with $\alpha_{0}=\lambda$, we have $\alpha_{1} \rightarrow\left(\omega_{1}, \omega_{1}\right)^{2}$. By Lemma 5 this leads to (101). 
THEOREM 43. If $r<s \leqq \beta_{0} ; \alpha \rightarrow\left(\beta_{0}\right)_{k}^{r} ; \beta_{1} \rightarrow(s)_{k}^{r}$, then

$$
\alpha \rightarrow\left(\beta_{0}, \beta_{1}\right)^{s} \text {. }
$$

This proposition remains valid if the types $\alpha, \beta_{0}, \beta_{1}$ are replaced by cardinals.

PRoof. Let $r<s \leqq \beta_{0} ; \alpha \rightarrow\left(\beta_{0}, \beta_{1}\right)^{s} ; \beta_{1} \rightarrow(s)_{k}^{r}$. We have to deduce that

$$
\alpha \rightarrow\left(\beta_{0}\right)_{k}^{r} \text {. }
$$

Let $\bar{S}=\alpha ;[S]^{r}=\sum^{\prime}[\nu<k] K_{\nu}$. Then $[S]^{s}=K_{0}^{\prime}+^{\prime} K_{1}^{\prime}$, where

$$
K_{0}^{\prime}=\sum[\nu<k]\left\{A: A \in[S]^{s} ; \quad[A]^{r} \subset K_{\nu}\right\} .
$$

Then there are $B C S ; \lambda<2$ such that $[B]^{\circ} \subset K_{\lambda}{ }^{\prime} ; \bar{B}=\beta_{\lambda}$. If $\lambda=1$, then $\bar{B} \rightarrow(s)_{k}^{r}$, and therefore there are $A \in[B]^{s} ; \nu<k$ such that $[A]^{r} \subset K_{\nu}$. Then $A \in K_{0}^{\prime} ; A \notin K_{1}^{\prime}$, which is false. Hence $\lambda=0$. Let $\{X, Y\}_{\neq}$ $C[B]$. Then we can write $X=\left\{x_{0}, \cdots, x_{r-1}\right\} ; Y=\left\{x_{m}, \cdots\right.$, $\left.x_{m+r-1}\right\}$, where $1 \leqq m \leqq r ;\left\{x_{0}, \cdots, x_{m+r-1}\right\}_{\neq}$. Put

$$
X_{\mu}=\left\{x_{\mu}, \cdots, x_{\mu+r-1}\right\} \quad(\mu \leqq m) .
$$

Now let $\mu<m$. Then, since $|B|=\left|\beta_{0}\right| \geqq s>r$, there is $Y_{\mu} \in[B]^{s}$ such that $X_{\mu}+X_{\mu+1} \subset Y_{\mu}$. But $Y_{\mu} \in K_{0}^{\prime}$, so that $X_{\mu}, X_{\mu+1} \in\left[Y_{\mu}\right]^{r} \subset K_{\nu_{\mu}}$, for some $\nu_{\mu}<k$. Then $\nu_{0}=\nu_{1}=\cdots=\nu_{m-1} ; X=X_{0} \in K_{\nu_{0}} ; \quad Y=X_{m}$ $\in K_{\nu_{m-1}}=K_{\nu_{0}}$. Since $X$ and $Y$ are arbitrary, it follows that $\nu_{0}$ is independent of $X$ and $Y$, and that $[B]^{r} \subset K_{\nu_{0}}$. This proves (102). The analogous theorem, with cardinals in place of types, is proved by means of the obvious modifications of the above argument.

Applications of Theorem 43. (a) Let $\lambda$ be the type of the continuum and $|\lambda|=\boldsymbol{\aleph}_{n}$. Then, by Theorem $30, \boldsymbol{\aleph}_{n} \rightarrow\left(\boldsymbol{\aleph}_{1}\right)_{2}^{2}$. Also, as is easily verified,

$$
6 \rightarrow(3)_{2}^{2} \text {. }
$$

Hence, by Theorem $43, \boldsymbol{\aleph}_{n} \rightarrow\left(\boldsymbol{\aleph}_{1}, 6\right)^{3}$, and therefore $\omega_{n} \rightarrow\left(\omega_{1}, 6\right)^{3}$. Now, by Theorem $15, \omega_{n} \rightarrow\left(\omega_{1}, r+3\right)^{r}(r \geqq 3)$ follows and therefore, finally,

$$
2 \aleph_{0} \rightarrow\left(\boldsymbol{N}_{1}, r+3\right)^{r} \quad(r \geqq 3) .
$$

(b) By (97),

$$
\omega_{1} \rightarrow\left(\omega_{0}+2\right)_{2}^{3} \text {. }
$$

By (103) and Theorem 39, we have

$$
m \rightarrow(4)_{2}^{8} \text {, }
$$

where $m=1+\sum[\mu<6] 2^{\mu^{2}}<2^{26}$. It now follows from (105) and (106), 
by Theorem 43 , that $\omega_{1} \rightarrow\left(\omega_{0}+2,2^{26}\right)^{4}$ and therefore, by Theorem 15 , that

$$
\omega_{1} \rightarrow\left(\omega_{0}+2,2^{26}+r-4\right)^{r} \quad(r \geqq 4) .
$$

We now give a new proof of the theorem of Dushnik and Miller [2]. ${ }^{11}$ Our proof bears some resemblance to the original proof but can, we think, be followed more easily.

TheOREM 44. If $a \geqq \boldsymbol{\aleph}_{0}$, then $a \rightarrow\left(\boldsymbol{\aleph}_{0}, a\right)^{2}$.

Proof. We use induction with respect to $a$. By Theorem 1, the assertion is true for $a=\boldsymbol{\aleph}_{0}$. We assume that $n>0$ and that the assertion is true for $a<\mathbf{\aleph}_{n}$, and we let

$$
|S|=b=\boldsymbol{\aleph}_{n}>\boldsymbol{\aleph}_{0} ; \quad[S]^{2}=K_{0}+K_{1} .
$$

We suppose that

$$
\text { if } X \in[S]^{\aleph_{0},} \text { then }[X]^{2} \nsubseteq K_{0},
$$

and we want to find $Y \in[S]^{b}$ such that $[Y]^{2} \subset K_{1}$.

There is a maximal set $A=\left\{x_{\nu}: \nu<l\right\} \subset S$ such that $l<\omega_{0},{ }^{12}$

$$
\begin{aligned}
x_{\nu} & \in U_{0}\left(x_{0}, \cdots, x_{\nu-1}\right), \\
\left|U_{0}\left(x_{0}, \cdots, x_{\nu}\right)\right| & =b \quad(\nu<l) .
\end{aligned}
$$

For, the relations (108) imply that $[A]^{2} \subset K_{0}$. Put $B=U_{0}(A)$. Then

$$
\left|B U_{0}(x)\right|<|B|=b \quad(x \in B) .
$$

Case 1. $b^{\prime}=b$. Then we define $x_{\nu}\left(\nu<\omega_{n}\right)$ as follows. Let $\nu<\omega_{n}$, and suppose that $x_{\mu} \in B(\mu<\nu)$. Then, by (109),

$$
\left|\sum[\mu<\nu]\left(\left\{x_{\mu}\right\}+B U_{0}\left(x_{\mu}\right)\right)\right|<|B|,
$$

and therefore there is $x_{\nu} \in B-\sum[\mu<\nu]\left(\left\{x_{\mu}\right\}+U_{0}\left(x_{\mu}\right)\right)$. We may put $Y=\left\{x_{\nu}: \nu<\omega_{n}\right\}$.

Case 2. $b^{\prime}=\boldsymbol{\aleph}_{m}<b$. Then $b=\sum\left[\mu<\omega_{m}\right] b_{\mu}$, where $b_{\mu}<b$. Let $x \in B$. Then there is a first ordinal $\rho(x)<\omega_{m}$ such that $\mid B U_{0}(x)<b_{\rho(x)}$, since otherwise we would obtain the contradiction $b=\sum\left[\mu<\omega_{m}\right] b_{\mu}$ $\leqq\left|B U_{0}(x)\right| \aleph_{m}<b$. Now put

$$
B(\tau)=\{x: x \in B ; \rho(x)=\tau\} \quad\left(\tau<\omega_{m}\right) .
$$

We define, by induction, $\tau_{\mu}, X_{\mu}\left(\mu<\omega_{m}\right)$ as follows. Let, for some $\nu<\omega_{m}$,

11 Theorem 3, (i).

${ }^{12}$ The symbol $U_{0}$ was defined in $\$ 2$. 


$$
\tau_{\mu}<\omega_{m} ; \quad X_{\mu} \in\left[B\left(\tau_{\mu}\right)\right]^{b_{\mu}} \quad(\mu<\nu) .
$$

Then, by definition of $m$,

$$
\begin{gathered}
\left|\sum\left[\mu<\nu ; x \in X_{\mu}\right]\left(\{x\}+B U_{0}(x)\right)\right| \leqq \sum\left[\mu<\nu ; x \in X_{\mu}\right]\left(1+b_{\tau_{\mu}}\right) \\
=\sum[\mu<\nu]\left(1+b_{\tau_{u}}\right) b_{\mu}<b .
\end{gathered}
$$

Hence $|D|=b$, where $D=B-\sum\left[\mu<\nu ; x \in X_{\mu}\right]\left(\{x\}+U_{0}(x)\right)$. There is a first ordinal $\tau_{\nu}<\omega_{m}$ such that $\left|D B\left(\tau_{\nu}\right)\right|<b_{\nu}$, since otherwise we would have the contradiction

$$
b=|D|=\sum\left[\mu<\omega_{m}\right]|D B(\mu)| \leqq b_{w} \aleph_{m}<b .
$$

Now we can choose $X_{\nu} \in\left[D B\left(\tau_{\nu}\right)\right]^{b_{\nu}}$. Then $X_{\nu} \subset U_{1}\left(X_{\mu}\right)\left(\mu<\nu<\omega_{m}\right)$. By the induction hypothesis there is $Y_{\mu} \in\left[X_{\mu}\right]^{b_{\mu}}$ such that $\left[Y_{\mu}\right]^{2} \subset K_{1}$ $\left(\mu<\omega_{m}\right)$. Then we may put $Y=\sum\left[\mu<\omega_{m}\right] Y_{\mu}$. This proves Theorem 44 .

Our next theorem may be considered as providing, in the case $r=1$, an extension of the Corollary of Theorem 39 to the case of arbitrary types $\beta_{\nu}$, not necessarily ordinals. In view of (5), the extension to values $r \geqq 2$ is false.

Let $k>0$, and consider any types $\beta_{\nu}(\nu<k)$. Let $\bar{B}_{\nu}=\beta_{\nu}$, and denote by $P$ the cartesian product of the sets $B_{\nu}$, i.e. the set of all mappings $\nu \rightarrow x_{\nu} \in B_{\nu}$ defined for $\nu<k$. We order $P$ alphabetically and call the order type $\pi$ of $P$ the alphabetical product of the types $\beta_{\nu}$ and write

$$
\pi=\Pi \times[\nu<k] \beta_{\nu} .
$$

This multiplication has been considered by Hausdorff [10].

Theorem 45. If $k>0$, then $\Pi \times[\nu<k] \beta_{\nu} \rightarrow\left(\beta_{0}, \beta_{1}, \cdots\right)_{k}^{1}$.

PROoF. In spite of its somewhat complicated appearance the proof is, in fact, very simple, as can be seen by following it in the case $k=2$ or $k=3$. Let $P=\sum[\nu<k] K_{\nu}$. We want to find $X \subset P$ and $\nu_{0}<k$ such that $\bar{X}=\beta_{\nu_{0}} ; X \subset K_{\nu_{0}}$. We use the notation $\left(z_{0}, z_{1}, \cdots, \widehat{z}_{n}\right)$ for the system of all $z_{\nu}$ such that $\nu<n$, ordered according to increasing $\nu$. Thus

$$
P=\left\{\left\{x_{0}, x_{1}, \cdots, \widehat{x}_{k}\right\}: x_{\nu} \in B_{\nu} \text { for } \nu<k\right\} .
$$

Case 1. There is $\nu<k$ such that the following condition is satisfied. There is a system of elements $x_{\mu} \in B_{\mu}(\mu<\nu)$ such that, given any $x_{\nu} \in B_{\nu}$, there is some system $z\left(x_{\nu}\right)=\left(x_{0}, x_{1}, \cdots, \hat{x}_{k}\right)$ which belongs to $K_{\nu}$. Then we may put $X=\left\{z(x): x \in B_{\nu}\right\}$.

Case 2. There is no $\nu$ such that the condition of Case 1 holds. Then, given any $\nu<k$ and any elements $x_{\mu} \in B_{\mu}(\mu<\nu)$, there is a 
function $f_{\nu}\left(x_{0}, x_{1}, \cdots, \hat{x}_{\nu}\right) \in B_{\nu}$ such that, for any choice of $x_{\lambda} \in B_{\lambda}$ $(\nu<\lambda<k)$,

$$
\left(x_{0}, x_{1}, \cdots, \hat{x}_{\nu}, f_{\nu}\left(x_{0}, \cdots, \hat{x}_{\nu}\right), x_{\nu+1}, \cdots, \hat{x}_{k}\right) \notin K_{\nu} .
$$

In particular, the function $f_{0}\left(\hat{x}_{0}\right)$ is constant. Then we define, inductively, elements $y_{\nu}(\nu<k)$ as follows. We put, for $\nu<k, y_{\nu}$ $=f_{\nu}\left(y_{0}, \cdots, y_{\nu}\right)$. Then $y_{\mu} \in B_{\mu}(\mu<k)$, and hence there is $\nu<k$ such that $\left(y_{0}, \cdots, y_{k}\right) \in K_{\nu}$. But then

$$
\left(y_{0}, y_{1}, \cdots, \hat{y}_{v}, f_{v}\left(y_{0}, \cdots, \hat{y}_{v}\right), y_{v+1}, \cdots, \hat{y}_{k}\right) \in K_{v}
$$

which contradicts the definition of $f_{v}$. The theorem is proved.

8. Canonical partition relations. Let $S$ be an ordered set, and consider a partition

$$
[S] r=\sum^{\prime}[\nu<k] K_{\nu} .
$$

To every such disjoint partition there belongs an equivalence relation $\Delta$ on $[S]^{r}$ defined by the rule that elements $X, Y$ of $[S]^{r}$ are equivalent for $\Delta$, in symbols:

$$
X \equiv Y(\cdot \Delta)
$$

if, and only if, there is $\nu<k$ such that $X, Y \in K_{\nu}$. This equivalence relation $\Delta$ is unaltered if the classes $K_{\nu}$ are renumbered in any way. The partition (110) and the corresponding equivalence relation $\Delta$ is called canonical ${ }^{13}$ if there is a system $\left(\epsilon_{0}, \epsilon_{1}, \cdots, \epsilon_{r-1}\right)$ of numbers $\epsilon_{\rho}<2$ such that, for $X=\left\{x_{0}, \cdots, x_{r-1}\right\}<C S ; Y=\left\{y_{0}, \cdots, y_{r-1}\right\}_{<}$ $C S$, we have $X \equiv Y(\cdot \Delta)$ if, and only if, $x_{\rho}=y_{\rho}$ for every $\rho<r$, such that $\epsilon_{p}=1$.

The canonical equivalence relation defined by means of the numbers $\epsilon_{\rho}$ is denoted by $\Delta_{e_{0} \epsilon_{1}}^{r} \cdots \epsilon_{r-1}$. The canonical partition relation

$$
\alpha \rightarrow *(\beta)^{r}
$$

has, by definition, the following meaning. Whenever $\bar{S}=\alpha$, and (110) is any disjoint partition, with any arbitrary $k$, then there is $B \subset S$ such that $\bar{B}=\beta$, and such that the equivalence relation $\Delta$ belonging to $(110)$, if restricted to $[B]^{r}$, coincides with some canonical equivalence relation $\Delta_{\epsilon_{0}}^{r} \cdots \epsilon_{r-1}$. The main result of [4] is expressible in the form $\omega_{0} \rightarrow *\left(\omega_{0}\right)^{r}$. The problem arises of finding canonical partition relations between types other than $\omega_{0}$. The main difference between canonical and noncanonical relations derives from the fact that if the

${ }^{13}[4 ; 5]$. The notation used in the present note differs slightly from that used in the earlier papers. 
canonical relation (111) holds then a certain choice of a subset of $S$ can be made irrespective of the number $|k|$ of classes of (110). The relation $\omega_{0} \rightarrow *\left(\omega_{0}\right)^{1}$ is equivalent to the statement that, if $a$ denumerable set $S$ is arbitrarily split into nonoverlapping subsets $S_{\nu}$, then there are either infinitely many nonempty subsets $S_{\nu}$, or else at least one of the subsets $S_{\nu}$ is infinite.

The following theorem establishes a connection between canonical and noncanonical partition relations.

THEOREM 46. (i) Let $q_{s}$ denote the number of distinct equivalence relations which can be defined on the set $[0, s]$. Let

$$
s=\left(\begin{array}{c}
2 r \\
r
\end{array}\right) ; \quad|\beta|>2 r ; \quad \alpha \rightarrow(\beta)_{q_{a}}^{2 r} .
$$

Then $\alpha \rightarrow *(\beta)^{r}$. If $|\beta|>4 ; \alpha \rightarrow(\beta)_{203}^{4}$, then $\alpha \rightarrow *(\beta)^{2}$.

(ii) If $m, r \geqq 0$, and $2^{\aleph_{\nu}} \leqq \boldsymbol{\aleph}_{n}$ for $m \leqq n<m+2 r+1 ; \nu<n$, then $\omega_{m+2 r+1} \rightarrow *\left(\omega_{m}+2 r+1\right)^{r+1}$.

REMARK. The first few values of $q_{s}$ are:

$q_{0}=1 ; \quad q_{1}=1 ; \quad q_{2}=2 ; \quad q_{3}=5 ; \quad q_{4}=15 ; \quad q_{5}=52 ; \quad q_{6}=203$.

A rough estimate for all $s$ is

$$
q_{s} \leqq 2^{\left(\begin{array}{l}
s \\
2
\end{array}\right),}
$$

obtained by observing that an equivalence relation is fixed if for $\mu<\nu<s$ it is decided whether $\mu \equiv \nu$ or $\mu \neq \nu$. It is easy to prove that, for $s>0$,

$$
q_{s+1}=\left(\begin{array}{l}
s \\
0
\end{array}\right) q_{s}+\left(\begin{array}{l}
s \\
1
\end{array}\right) q_{s-1}+\cdots+\left(\begin{array}{l}
s \\
s
\end{array}\right) q_{0}
$$

and hence $q_{s} \leqq s !$.

Deduction of (ii) from (i). By Theorem 39 (iii), we have

$$
\omega_{m+2 r+1} \rightarrow\left(\omega_{m}+2 r+1\right)_{k}^{2 r+2} \quad\left(k<\omega_{0}\right),
$$

and the conclusion follows from Theorem 46 (i).

Proof of (i). Suppose that (112) holds. Let $\bar{S}=\alpha$, and consider any disjoint partition (110). Let $\Delta$ be the equivalence relation on $[S]^{r}$ which belongs to (110). Our first aim is to define a certain equivalence relation $\Delta^{*}$ on $[S]^{2 r}$.

Let $[[0,2 r]] r=\left\{P_{0}, P_{1}, \cdots, P_{s-1}\right\}_{\star}$. Then

$$
s=\left(\begin{array}{c}
2 r \\
r
\end{array}\right) \text {. }
$$


Let $X=\left\{x_{0}, \cdots, x_{2 r-1}\right\}<C S$. Define the equivalence relation $\Delta(X)$ on $[0, s]$ by putting, for $\mu<\nu<s$,

$$
\mu \equiv \nu(\cdot \Delta(X))
$$

if, and only if, $\left\{x_{\lambda}: \lambda \in P_{\mu}\right\} \equiv\left\{x_{\lambda}: \lambda \in P_{\nu}\right\}(\cdot \Delta)$. Put, for $X, Y \in[S]^{2 r}$,

$$
X \equiv Y\left(\cdot \Delta^{*}\right)
$$

if, and only if, $\Delta(X)=\Delta(Y)$.

Now, by definition of $q_{s}, \Delta^{*}$ has at most $q_{s}$ nonempty classes. By (112), there is $B C S$ such that $\bar{B}=\beta$, and any two elements of $[B]^{2 r}$ are equivalent for $\Delta^{*}$. This means that, in the terminology of [4], $\Delta$ is invariant in $[B]^{r}$ (cf. $[4$, p. 253]). Choose any $A \subset B$ such that $2 r<|A|<\boldsymbol{\aleph}_{0}$, which is possible since $|\beta|>2 r$. Then $\Delta$ is invariant in $[A]^{r}$ and hence, by $\left[4\right.$, Theorem 2], canonical in $[A]^{r}$. Thus there is a canonical equivalence relation $\Delta(A)$ on $[B]^{r}$ such that $\Delta=\Delta(A)$ on $[A]^{r}$. It only remains to show that $\Delta(A)$ is independent of $A$.

Let $A_{0}, A_{1} \subset B ; 2 r<\left|A_{0}\right|,\left|A_{1}\right|<\boldsymbol{N}_{0}$, and let $\Delta=\Delta\left(A_{0}\right)=\Delta_{\epsilon_{0}}^{r} \cdots \epsilon_{r-1}$ on $\left[A_{0}\right]^{r}, \Delta=\Delta\left(A_{1}\right)=\Delta_{\eta_{0}}^{r} \cdots \eta_{r-1}$ on $\left[A_{1}\right]^{r}$. Then $2 r<\left|A_{0}+A_{1}\right|<\boldsymbol{N}_{0}$, and hence, for some $\kappa_{\rho}<2, \Delta=\Delta\left(A_{0}+A_{1}\right)=\Delta_{\kappa_{0}}^{r} \cdots \kappa_{r-1}$ on $\left[A_{0}+A_{1}\right]^{r}$. Let $A_{0}=\left\{y_{0}, y_{1}, \cdots, y_{2 r-1}, \cdots\right\}<$. Consider the sets

$$
P=\left\{y_{2 \lambda}: \lambda<r\right\} ; \quad Q=\left\{y_{2 \lambda+1-\epsilon \lambda}: \lambda<r\right\} .
$$

By definition of $\Delta_{\epsilon_{0}}^{r} \cdots \epsilon_{r-1}$, we have $P \equiv Q\left(\cdot \Delta_{\epsilon_{0}}^{r} \cdots \epsilon_{r-1}\right)$. Hence

$$
P \equiv Q(\cdot \Delta) ; \quad P \equiv Q\left(\cdot \Delta_{\kappa_{0}}^{r} \cdots \kappa_{r}-1\right) ; \quad \kappa_{\rho} \leqq \epsilon_{\rho}(\rho<r) .
$$

Similarly, by considering the sets $P$ and $Q^{\prime}=\left\{y_{2 \lambda+1-n_{\lambda}}: \lambda<r\right\}$, we find that $P \equiv Q^{\prime}\left(\cdot \Delta_{\kappa_{0}}^{r} \cdots \kappa_{r-1}\right) ; P \equiv Q^{\prime}(\cdot \Delta)$;

$$
P \equiv Q^{\prime}\left(\cdot \Delta_{\epsilon_{0} \cdots \epsilon_{r-1}}^{r}\right) ; \quad \epsilon_{\rho} \leqq \kappa_{\rho}(\rho<r) .
$$

Hence $\epsilon_{\rho}=\kappa_{\rho}$ for all $\rho$. For reasons of symmetry, $\eta_{\rho}=\kappa_{\rho}$, and so, finally $\epsilon_{\rho}=\eta_{\rho}(\rho<r)$. Therefore $\Delta(A)$ is independent of $A$, which means that $\Delta$ is canonical in the whole set $[B]^{r}$. This proves Theorem 46.

9. Polarised partition relations. The relation $a \rightarrow\left(b_{0}, b_{1}\right)^{2}$ refers to a partition of the set of all pairs of elements of a set $S$ of cardinal $a$. Instead of pairs of elements of one and the same set we shall now consider pairs of elements, one from each of two sets $S_{0}, S_{1}$. The relation $a \rightarrow\left(b_{0}, b_{1}\right)^{2}$ can be thought of as referring, in the terminology of combinatorial graph theory (linear combinatorial topology), to decompositions of the complete graph of cardinal $a$ into two subgraphs. The new kind of relation to be defined now refers to decom- 
positions of the "complete" even graph of cardinal-pair $a_{0}, a_{1}$, i.e. the graph obtained by joining every "point" of a set of cardinal $a_{0}$ to every point of a disjoint set of cardinal $a_{1}$.

More generally, we introduce the notation

$$
\left[S_{0}, S_{1}, \cdots, S_{t-1}\right]^{r_{0}, r_{1}, \cdots, r_{t-1}}
$$

for the cartesian product of the $t$ sets $\left[S_{\lambda} \mid{ }_{\lambda}\right.$, i.e. we put

$$
\left[S_{0}, \cdots, S_{t-1}\right]^{r_{0}}, \cdots, r_{t-1}=\left\{\left(X_{0}, \cdots, X_{t-1}\right): X_{\lambda} \in\left[S_{\lambda}\right]^{\lambda} \text { for } \lambda<t\right\} .
$$

We shall always have $0<t<\omega_{0}$. The introduction of this set leads naturally to the following definition of a corresponding partition relation. The relation

$$
\left(\begin{array}{c}
a_{0} \\
a_{1} \\
\vdots \\
a_{t-1}
\end{array}\right) \rightarrow\left(\begin{array}{ccc}
b_{00} & b_{01} & \cdots \\
b_{10} & b_{11} & \ldots \\
\vdots & \vdots & \ldots \\
b_{t-1,0} & b_{t-1,1} & \cdots
\end{array}\right)_{k}
$$

has, by definition, the following meaning. Whenever $\left|S_{\lambda}\right|=a_{\lambda}$ for $\lambda<t$, and

$$
\left[S_{0}, \cdots, S_{t-1}\right]^{r_{0}, \ldots, r_{t-1}}=\sum[\nu<k] K_{\nu},
$$

then there are sets $B_{\lambda} \subset S_{\lambda}$ and an ordinal $\nu<k$ such that $\left|B_{\lambda}\right|=b_{\lambda \nu}$ for $\lambda<t$, and $\left[B_{0}, \cdots, B_{t-1}\right]^{r_{0}, \cdots, r_{t-1}} \subset K_{\nu}$. There is a similar kind of relation involving order types which will, however, not be considered here. If the number of classes is finite we write in the rows on the right hand side of (113) a last element. The negation of (113) is obtained by replacing $\rightarrow$ by $\rightarrow$. If, for $\lambda<t, b_{\lambda 0}=b_{\lambda 1}=\cdots$, we use the obvious abbreviation for (113). Cf. Theorem 49.

The passage from our former type of partition relation i.e. the case $t=1$, to the more general kind (113) bears a certain resemblance to the process of polarisation used in the theory of algebraic forms, which accounts for the name polarised partition relation suggested for relations of the form (113).

We shall deduce some results for polarised relations but will not develop the theory to the same extent as was done in the case of the nonpolarised relation.

In [11] one of us has considered polarised canonical parition relations between finite numbers, and also involving $\omega_{0}$.

THEOREM 47. If $a^{\prime}=b^{\prime}$, then 


$$
\left(\begin{array}{l}
a \\
b
\end{array}\right) \rightarrow\left(\begin{array}{ll}
a & 1 \\
1 & b
\end{array}\right)^{1,1} .
$$

In particular, (114) holds if $1<a, b<\boldsymbol{\aleph}_{0}$.

THEOREM 48. The relation

$$
\left(\begin{array}{c}
\boldsymbol{N}_{0} \\
b
\end{array}\right) \rightarrow\left(\begin{array}{ll}
\boldsymbol{N}_{0} & \boldsymbol{\aleph}_{0} \\
\boldsymbol{N}_{0} & b
\end{array}\right)^{1,1}
$$

holds if, and only if, either $b=0$ or $b^{\prime}>\boldsymbol{\aleph}_{0}$. In particular,

$$
\left(\begin{array}{l}
\aleph_{0} \\
2 \aleph_{0}
\end{array}\right) \rightarrow\left(\begin{array}{ll}
\aleph_{0} & \aleph_{0} \\
\aleph_{0} & 2 \aleph_{0}
\end{array}\right)^{1,1}
$$

Proof of Theorem 47. If $1<a<\boldsymbol{\aleph}_{0}$, then $b^{\prime}=a^{\prime}=2 ; 1<b<\boldsymbol{\aleph}_{0}$. Let, in this case, $A=[0, a] ; B=[0, b]$. Then $[A, B]^{1,1}=K_{0}+^{\prime} K_{1}$, where

$$
K_{0}=\{(\kappa, \lambda): \kappa<a ; \lambda<b ; \kappa+\lambda \text { even }\} .
$$

Then, for any $\kappa<a$, the pairs $(\kappa, 0)$ and $(\kappa, 1)$ lie in different classes $K_{\nu}$, and the same holds for $(0, \lambda)$ and $(1, \lambda)$. This proves (114). Now let $a \geqq \boldsymbol{\aleph}_{0}$. Then $a^{\prime}=b^{\prime}=\boldsymbol{\aleph}_{n}$, say, and we can write

$$
a=\sum\left[\nu<\omega_{n}\right] a_{\nu} ; \quad b=\sum\left[\nu<\omega_{n}\right] b_{\nu},
$$

where $a_{\nu}<a ; b_{\nu}<b$. Let $A=\sum^{\prime}\left[\nu<\omega_{n}\right] A_{\nu} ; B=\sum^{\prime}\left[\nu<\omega_{n}\right] B_{\nu}$; $\left|A_{\nu}\right|=a_{\nu} ;\left|B_{\nu}\right|=b_{\nu}$. Then $[A, B]^{1,1}=K_{0}+K_{1}$, where

$$
\begin{array}{lll}
K_{0}=\left\{(x, y): x \in A_{\mu} ;\right. & y \in B_{\nu} ; & \left.\mu<\nu<\omega_{n}\right\}, \\
K_{1}=\left\{(x, y): x \in A_{\mu} ;\right. & y \in B_{\nu} ; & \left.\nu \leqq \mu<\omega_{n}\right\} .
\end{array}
$$

Let $X \in[A]^{a} ; y_{0} \in B$. Then $y_{0} \in B_{\nu}$ for some $\nu<\omega_{n}$. We have $\left|A_{\mu} X\right|$ $\leqq\left|A_{\mu}\right|<a$ and hence, by definition of $n, \sum[\mu \leqq \nu]\left|A_{\mu} X\right|<a=|X|$. Therefore we can choose $x_{0} \in X-\sum[\mu \leqq \nu] A_{\mu}$. Then $\left(x_{0}, y_{0}\right) \notin K_{0}$, and and hence $\left[X,\left\{y_{0}\right\}\right]^{1,1} \Phi ; K_{0}$. By symmetry, it follows that, if $Y \in[B]^{b}$; $x_{1} \in A$, then $\left[\left\{x_{1}\right\}, Y\right]^{1,1} \nsubseteq K_{1}$. This proves (114) and completes the proof of Theorem 47.

Proof of Theorem 48. If $b=0$, then (115) obviously holds. If $0<b<\boldsymbol{\aleph}_{0}$, then (115) is false, as is seen by considering the partition of the set in question in which $K_{1}=0$. Next, if $b^{\prime}=\boldsymbol{\aleph}_{0}^{\prime}$, then, by Theorem 47 ,

$$
\left(\begin{array}{c}
\aleph_{0} \\
b
\end{array}\right) \rightarrow\left(\begin{array}{ll}
\aleph_{0} & 1 \\
1 & b
\end{array}\right)^{1,1}
$$


Hence, a fortiori, (115) is false. It remains to prove (115) under the assumption $b^{\prime}>\boldsymbol{\aleph}_{0}$.

Let $|A|=\boldsymbol{\aleph}_{0} ;|B|=b ;[A, B]^{1,1}=K_{0}+K_{1}$. We may suppose that

$$
(X, Y) \in[A, B]^{\aleph_{0}, b} \text { implies }[X, Y]^{1,1} \nsubseteq K_{1} \text {. }
$$

Let $(X, Y) \in[A, B]^{\aleph_{0}, b}$.

1. Put $Y_{0}=\sum[x \in X]\left\{y:(x, y) \in K_{0}\right\}$. Then $\left[X, Y=Y_{0}\right]^{1,1} \subset K_{1}$, and hence, by (117), $\left|Y-Y_{0}\right|<b$,

$$
\sum[x \in X]\left|\left\{y: y \in Y ;(x, y) \in K_{0}\right\}\right| \geqq\left|Y Y_{0}\right|=b .
$$

Since $b^{\prime}>\boldsymbol{\aleph}_{0}=|X|$, this implies the existence of $x_{0} \in X$ such that

$$
\left|\left\{y: y \in Y ;\left(x_{0}, y\right) \in K_{0}\right\}\right|=b .
$$

Put

$$
\phi(X, Y)=x_{0} ; \quad \psi(X, Y)=\left\{y: y \in Y ;\left(x_{0}, y\right) \in K_{0}\right\} .
$$

Then

$$
\begin{gathered}
\phi(X, Y) \in X ; \quad \psi(X, Y) \in[Y]^{b}, \\
{[\{\phi(X, Y)\}, \psi(X, Y)]^{1,1} \subset K_{0} .}
\end{gathered}
$$

2. Put $f(y)=\left\{x: x \in X ;(x, y) \in K_{0}\right\} \quad(y \in Y)$. If

$$
y \in Y \text { implies }|f(y)|<\boldsymbol{\aleph}_{0},
$$

then $b=|Y|=\sum\left[P \subset X ;|P|<\boldsymbol{\aleph}_{0}\right]|\{y: y \in Y ; f(y)=P\}|$. But, since there are only $\boldsymbol{\aleph}_{0}$ distinct sets $P$, and $b^{\prime}>\boldsymbol{\aleph}_{0}$, there is $P_{1} \subset X$ such that $\left|P_{1}\right|<\boldsymbol{\aleph}_{0} ;\left|Y_{1}\right|=b$, where $Y_{1}=\left\{y: y \in Y ; f(y)=P_{1}\right\}$. Then $\left[X-P_{1}\right.$, $\left.Y_{1}\right]^{1,1} \subset K_{1}$, which contradicts (117). Therefore (118) is false, and there is $y_{1} \in Y$ such that $\left|f\left(y_{1}\right)\right|=\boldsymbol{\aleph}_{0}$. Put $\phi_{1}(X, Y)=y_{1} ; \psi_{1}(X, Y)$ $=f\left(y_{1}\right)$. Then $\phi_{1}(X, Y) \in Y ; \psi_{1}(X, Y) \in[X]^{\aleph_{0}}$;

$$
\left[\psi_{1}(X, Y),\left\{\phi_{1}(X, Y)\right\}\right]^{1,1} \subset K_{0} .
$$

3. We define sequences $x_{\nu}, y_{\nu}, X_{\nu}, Y_{\nu}\left(\nu<\omega_{0}\right)$ as follows.

$x_{0}=\phi(A, B) ; Y_{0}=\psi(A, B) ; y_{0}=\phi_{1}\left(A-\left\{x_{0}\right\}, Y_{0}\right) ; X_{0}=\psi_{1}\left(A-\left\{x_{0}\right\}, Y_{0}\right)$.

For $0<\nu<\omega_{0}$, we put

$$
\begin{aligned}
& x_{\nu}=\phi\left(X_{\nu-1}, Y_{\nu-1}-\left\{y_{\nu-1}\right\}\right) ; \quad Y_{\nu}=\psi\left(X_{\nu-1}, Y_{\nu-1}-\left\{y_{\nu-1}\right\}\right) ; \\
& y_{\nu}=\phi_{1}\left(X_{\nu-1}-\left\{x_{\nu}\right\}, Y_{\nu}\right) ; \quad X_{\nu}=\psi_{1}\left(X_{\nu-1}-\left\{x_{\nu}\right\}, Y_{\nu}\right) .
\end{aligned}
$$

Then

$$
\begin{aligned}
& x_{\nu} \in X_{\nu-1} \subset X_{\nu-2}-\left\{x_{\nu-1}\right\} \subset X_{\nu-3}-\left\{x_{\nu-2}, x_{\nu-1}\right\} \subset \cdots \\
& \subset X_{0}-\left\{x_{1}, \cdots, x_{\nu-1}\right\} \subset A-\left\{x_{0}, \cdots, x_{\nu-1}\right\}
\end{aligned}
$$




$$
\begin{aligned}
y_{\nu} \in Y_{\nu} \subset Y_{\nu-1}-\left\{y_{\nu-1}\right\} \subset \cdots \subset Y_{0}-\left\{y_{0}, \cdots, y_{\nu-1}\right\} \\
\subset B-\left\{y_{0}, \cdots, y_{\nu-1}\right\} ; \\
\quad\left[\left\{x_{\nu}\right\}, Y_{\nu}\right]^{1,1} \subset K_{0} ;\left[\left\{x_{\nu}\right\},\left\{y_{\nu}, y_{\nu+1}, \cdots\right\}\right]^{1,1} \subset K_{0} ; \\
\quad\left[X_{\nu},\left\{y_{\nu}\right\}\right]^{1,1} \subset K_{0} ;\left[\left\{x_{\nu+1}, x_{\nu+2}, \cdots\right\},\left\{y_{\nu}\right\}\right]^{1,1} \subset K_{0} ; \\
\quad\left(x_{\mu}, y_{\nu}\right) \in K_{0}\left(\mu, \nu<\omega_{0}\right) .
\end{aligned}
$$

This proves (115). Finally, as is well known [13, p. 135], $\left(2^{\boldsymbol{\aleph}_{0}}\right)^{\prime}>\boldsymbol{\aleph}_{0}$, so that (116) is a special case of (115). This proves Theorem 48.

We introduce the notation

$$
\left\{\begin{array}{l}
a \\
b
\end{array}\right\}=\left|[A]^{b}\right|
$$

where $A$ is a set such that $|A|=a$. If $a, b<\boldsymbol{N}_{0}$, then

$$
\left\{\begin{array}{l}
a \\
b
\end{array}\right\}
$$

is the ordinary binomial coefficient

$$
\left(\begin{array}{l}
a \\
b
\end{array}\right)
$$

The following lemma is probably well known.

Lemma 6. If $a \geqq \boldsymbol{\aleph}_{0}$, then

$$
\left\{\begin{array}{l}
a \\
b
\end{array}\right\}=a^{b} \text { for } b \leqq a \text { and }\left\{\begin{array}{l}
a \\
b
\end{array}\right\}=0 \text { for } b>a .
$$

Proof. The result is obvious for $b=0$ and for $b>a$. Now let $0<b<a$. Choose $n$ such that $|n|=b$, and $A_{\nu}, A$ such that $\left|A_{\nu}\right|=a$ for $\nu<n$, and $A=\sum^{\prime}[\nu<n] A_{\nu}$. Then $|A|=a b=a$. If $X \in[A]^{b}$, then $X=\left\{x_{\nu}: \nu<n\right\}$. Every $x_{\nu}$ has $a$ possible values. Hence

$$
\left\{\begin{array}{l}
a \\
b
\end{array}\right\} \leqq a^{b}
$$

On the other hand, if $y_{\nu} \in A_{\nu}$ for $\nu<n$, then $Y=\left\{y_{\nu}: \nu<n\right\} \in[A]^{b}$, and the set of all such $Y$ has a cardinal $a^{b}$. Hence

$$
\left\{\begin{array}{l}
a \\
b
\end{array}\right\} \geqq a^{b},
$$

and the lemma follows. 
Theorem 49. Suppose that $0<s<t<\boldsymbol{N}_{0} ; k>0$;

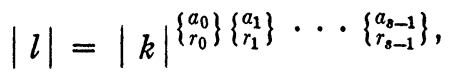$$
\left(\begin{array}{c}
a_{0} \\
\vdots \\
a_{s-1}
\end{array}\right) \rightarrow\left(\begin{array}{c}
b_{0} \\
\vdots \\
b_{s-1}
\end{array}\right)_{k}^{r_{0}, \cdots, r_{s-1}},
$$$$
\left(\begin{array}{c}
a_{s} \\
\dot{a_{t-1}} \\
\cdot \\
a_{t}
\end{array}\right) \rightarrow\left(\begin{array}{c}
b_{s} \\
\dot{b_{t-1}} \\
\dot{b_{t}}
\end{array}\right)_{l}^{r_{s}, \cdots, r_{t-1}}
$$

Then

$$
\left(\begin{array}{c}
a_{0} \\
\vdots \\
a_{t-1}
\end{array}\right) \rightarrow\left(\begin{array}{c}
b_{0} \\
\vdots \\
b_{t-1}
\end{array}\right)_{k}^{r_{0}, \cdots, r_{t-1}}
$$

Proof. We use the notation of the partition calculus explained in the proof of Theorem 25. In addition, if $\Delta$ is a partition of $M$, and $M^{\prime} \subset M$, then the relation

$$
|\Delta| \leqq a \text { in } M^{\prime}
$$

expresses the fact that the number of classes of $\Delta$ containing at least one element of $M^{\prime}$ is at most $a$. (119) and (120) imply that $b_{\lambda} \leqq a_{\lambda}$ for $\lambda<t$. If $b_{\lambda}<r_{\lambda}$ for some $\lambda<t$, then (121) holds trivially. Hence we may assume that

$$
r_{\lambda} \leqq b_{\lambda} \leqq a_{\lambda}
$$

for $\lambda<t$.

Let $\left|A_{\lambda}\right|=a_{\lambda}$ for $\lambda<t$, and consider any equivalence relation $\Delta$ on $\left[A_{0}, \cdots, A_{t-1}\right]^{r_{0}}, \ldots, r_{t-1}$ such that $|\Delta| \leqq|k|$. Our aim is to find $B_{\lambda} \in\left[A_{\lambda}\right]^{b_{\lambda}}(\lambda<t)$ such that

$$
|\Delta| \leqq 1 \text { in }\left[B_{0}, \cdots, B_{t-1}\right]^{r_{0}, \cdots, r_{t-1}} \text {. }
$$

Put, for $X_{\lambda} \in\left[A_{\lambda}\right]^{r \lambda}(s \leqq \lambda<t)$,

$$
\Delta_{1}\left(X_{s}, \cdots, X_{t-1}\right)=\prod \Delta\left(X_{0}, \cdots, X_{t-1}\right),
$$

where the last product is extended over all systems $\left(X_{0}, \cdots, X_{s-1}\right)$ $\in\left[A_{0}, \cdots, A_{s-1}\right]^{r_{0}}, \ldots, r_{s-1}$. By (122), this product has at least one factor. It follows that $\left|\Delta_{1}\right| \leqq|l|$. Hence, by (120), there is $B_{\lambda} \in\left[A_{\lambda}\right]^{b_{\lambda}}$ $(s \leqq \lambda<t)$ such that

$$
\left|\Delta_{1}\right| \leqq 1 \text { in }\left[B_{s}, \cdots, B_{t-1}\right]^{r_{s}}, \cdots, r_{t-1} .
$$


By (122), we can choose

$$
Y_{\lambda} \in\left[B_{\lambda}\right]^{\lambda} \quad(s \leqq \lambda<t) .
$$

Put, for $X_{\lambda} \in\left[A_{\lambda}\right]^{r \lambda}(\lambda<s)$,

$$
\Delta_{2}\left(X_{0}, \cdots, X_{s-1}\right)=\Delta\left(X_{0}, \cdots, X_{s-1}, Y_{s}, \cdots, Y_{t-1}\right) .
$$

Then $\left|\Delta_{2}\right| \leqq|k|$, and therefore, by (119), there is $B_{\lambda} \in\left[A_{\lambda}\right]_{\lambda}^{b}(\lambda<s)$ such that $\left|\Delta_{2}\right| \leqq 1$ in $\left[B_{0}, \cdots, B_{s-1}\right]^{r_{0}}, \ldots, r_{s-1}$. By $(122)$, we can choose $Y_{\lambda} \in\left[B_{\lambda}\right]^{r_{\lambda}}(\lambda<s)$. Then, for any $X_{\lambda} \in\left[B_{\lambda}\right]^{r_{\lambda}}(\lambda<t)$,

$$
\begin{aligned}
\left(X_{0}, \cdots, X_{t-1}\right) & \equiv\left(X_{0}, \cdots, X_{s-1}, Y_{s}, \cdots, Y_{t-1}\right) \\
& \equiv\left(Y_{0}, \cdots, Y_{s-1}, Y_{s}, \cdots, Y_{t-1}\right)(\cdot \Delta) .
\end{aligned}
$$

This proves (123) and so establishes Theorem 49.

We note the following special case of Theorem 49.

Corollary. If

then

$$
a_{0} \rightarrow\left(b_{0}\right)_{k}^{1} ; \quad a_{1} \rightarrow\left(b_{1}\right)_{|k|}^{1} a_{0}
$$

$$
\left(\begin{array}{l}
a_{0} \\
a_{1}
\end{array}\right) \rightarrow\left(\begin{array}{c}
b_{0} \\
b_{1}
\end{array}\right)_{k}^{1,1}
$$

We give some applications of this last result.

(a) If $0<d<\boldsymbol{N}_{0} \leqq a_{1}$, then

$$
\left(\begin{array}{c}
2 d-1 \\
a_{1}
\end{array}\right) \rightarrow\left(\begin{array}{l}
d \\
a_{1}
\end{array}\right)_{2}^{1,1}
$$

This is best possible in the sense that, if $2 d-1$ is replaced by $2 d-2$, the last relation becomes false. We even have, as is easily seen,

$$
\left(\begin{array}{c}
2 d-2 \\
a_{1}
\end{array}\right)+\left(\begin{array}{l}
d \\
1
\end{array}\right)_{2}^{1,1} \quad\left(0<d<\aleph_{0} \leqq a_{1}\right) .
$$

(b) If $a_{0}^{\prime}>|k|>0 ; a_{1}^{\prime}>|k|^{a_{0}}$, then

$$
\left(\begin{array}{l}
a_{0} \\
a_{1}
\end{array}\right) \rightarrow\left(\begin{array}{l}
a_{0} \\
a_{1}
\end{array}\right)_{k}^{1,1}
$$

In particular, if we assume that $2 \boldsymbol{\aleph}_{0}=\boldsymbol{\aleph}_{1}$, then

$$
\left(\begin{array}{l}
N_{0} \\
N_{2}
\end{array}\right) \rightarrow\left(\begin{array}{l}
N_{0} \\
N_{2}
\end{array}\right)_{2}^{1,1}
$$


More generally, if $2 \boldsymbol{\aleph}_{n}=\aleph_{n+1}$, then

$$
\left(\begin{array}{c}
\boldsymbol{N}_{n} \\
\boldsymbol{N}_{n+2}
\end{array}\right) \rightarrow\left(\begin{array}{c}
\boldsymbol{N}_{n} \\
\boldsymbol{N}_{n+2}
\end{array}\right)_{2}^{1,1}
$$

This is best possible in the following strong sense.

(c) If $a^{\prime} \leqq|k| ; b>0$, then

$$
\left(\begin{array}{l}
a \\
b
\end{array}\right)+\left(\begin{array}{l}
a \\
1
\end{array}\right)_{k}^{1,1} .
$$

To prove (c), choose $n \leqq k$ such that $|n|=a^{\prime}$. Then $a=\sum[\nu<n] a_{\nu}$, where $a_{\nu}<a$. Choose sets $A_{\nu}, A, B$ such that $\left|A_{\nu}\right|=a_{\nu}(\nu<n)$; $A=\sum^{\prime}[\nu<n] A_{\nu} ;|B|=b$, and put $[A, B]^{1,1}=\sum^{\prime}[\nu<k] K_{\nu}$, where $K_{\nu}=\left[A_{\nu}, B\right]^{1,1}(\nu<n)$. If, now, $0 \neq X \subset A ; y \in B ;[X,\{y\}]^{1,1} \subset K_{\nu}$ for some $\nu<k$, then $\nu<n ; X \subset A_{\nu} ;|X|<a$. This proves (c).

The following theorem is a corollary of a result due to Sierpiński.

THEOREM 50. If $2 \boldsymbol{\aleph}_{0}=\aleph_{1}$, then

$$
\left(\begin{array}{l}
N_{0} \\
N_{1}
\end{array}\right) \rightarrow\left(\begin{array}{l}
N_{0} \\
N_{1}
\end{array}\right)_{2}^{1,1}
$$

Proof. Let $A=\left[0, \omega_{0}\right] ; B=\left[0, \omega_{1}\right]$. According to Sierpinski ${ }^{14}$ the assumption $2 \boldsymbol{\aleph}_{0}=\boldsymbol{\aleph}_{1}$ implies, and is, in fact, equivalent to, the existence of a sequence of functions $f_{\lambda}(y) \in B(\lambda \in A)$, defined for $y \in B$, such that, given any $Y \in[B]^{\aleph_{1}}$, there is $\lambda_{0} \in A$ such that $\left\{f_{\lambda}(y): y \in Y\right\}=B$ $\left(\lambda_{0} \leqq \lambda<\omega_{0}\right)$. Then $[A, B]^{1,1}=K_{0}+^{\prime} K_{1}$, where $K_{0}=\{(\lambda, y): \lambda \in A$;

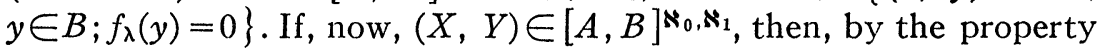
of the functions $f_{\lambda}$, there is $\lambda \in X ; y_{0}, y_{1} \in Y$ such that $f_{\lambda}\left(y_{\nu}\right)=\nu(\nu<2)$. Then $\left(\lambda, y_{\nu}\right) \in[X, Y]^{1,1} K_{\nu}(\nu<2)$. This proves the assertion.

THEOREM 51. If $a, b>1$;

$$
\left(\begin{array}{l}
a \\
b
\end{array}\right) \rightarrow\left(\begin{array}{l}
a \\
b
\end{array}\right)_{k}^{1,1} .
$$

Then

$$
\left(\begin{array}{l}
a^{\prime} \\
b^{\prime}
\end{array}\right) \rightarrow\left(\begin{array}{l}
a^{\prime} \\
b^{\prime}
\end{array}\right)_{k}^{1,1} .
$$

Proof. Let $l$ and $m$ be such that $|l|=a^{\prime} ;|m|=b^{\prime}$. Then $a=\sum[\lambda<l] a_{\lambda} ; b=\sum[\mu<m] b_{\mu}$, where $a_{\lambda}<a ; b_{\mu}<b$. Put $A^{\prime}=[0, l]$; $B^{\prime}=[0, m]$, and suppose that $\left[A^{\prime}, B^{\prime}\right]^{1,1}=\sum[\nu<k] K_{\nu}^{\prime}$. Then we choose sets $A_{\lambda}, A, B_{\mu}, B$ such that $\left|A_{\lambda}\right|=a_{\lambda}(\lambda<l) ;\left|B_{\mu}\right|=b_{\mu}(\mu<m)$;

14 [14], French translation in [16]. See also [1]. 
$A=\sum^{\prime}[\lambda<l] A_{\lambda} ; B=\sum^{\prime} \quad[\mu<m] B_{\mu}$. Then $|A|=a ; \quad|B|=b ;$ $[A, B]^{1,1}=\sum[\nu<k] K_{\nu}$, where $K_{\nu}=\left\{(x, y): x \in A_{\lambda} ; y \in B_{\mu} ;(\lambda, \mu)\right.$ $\left.\in K_{\nu}^{\prime}\right\}(\nu<k)$. By hypothesis, there is $(X, Y) \in[A, B]^{a, b}$ and $\nu<k$ such that $[X, Y]^{1,1} \subset K_{\nu}$. Put

$$
A^{\prime \prime}=\left\{\lambda: \lambda<l ; A_{\lambda} X \neq 0\right\} ; \quad B^{\prime \prime}=\left\{\mu: \mu<m ; B_{\mu} Y \neq 0\right\} .
$$

Then $a=|X|=\sum\left[\lambda \in A^{\prime \prime}\right]\left|A_{\lambda} X\right| ;\left|A_{\lambda} X\right| \leqq\left|A_{\lambda}\right|<a$. Hence, by definition of $a^{\prime},\left|A^{\prime \prime}\right| \geqq a^{\prime}$. But, $\left|A^{\prime \prime}\right| \leqq|l|=a^{\prime}$. Hence $\left|A^{\prime \prime}\right|=a^{\prime}$ and, by symmetry, $\left|B^{\prime \prime}\right|=b^{\prime}$.

Let $\lambda \in A^{\prime \prime} ; \mu \in B^{\prime \prime}$. Then we can choose $x \in A_{\lambda} X ; y \in B_{\mu} Y$, and we then have $(x, y) \in[X, Y]^{1,1} \subset K_{\nu} ;(\lambda, \mu) \in K_{\nu}^{\prime}$. Hence $\left[A^{\prime \prime}, B^{\prime \prime}\right]^{1,1}$ $\subset K_{\nu}^{\prime} ;\left(A^{\prime \prime}, B^{\prime \prime}\right) \in\left[A^{\prime}, B^{\prime}\right]^{a^{\prime}, b^{\prime}}$, and Theorem 51 follows.

Corollary. If $a>1$, then

For, if

$$
\left(\begin{array}{l}
a^{\prime} \\
a
\end{array}\right) \rightarrow\left(\begin{array}{l}
a^{\prime} \\
a
\end{array}\right)_{2}^{1,1}
$$

$$
\left(\begin{array}{l}
a^{\prime} \\
a
\end{array}\right) \rightarrow\left(\begin{array}{l}
a^{\prime} \\
a
\end{array}\right)_{2}^{1,1}
$$

then, by Theorem 51 and the known equation $a^{\prime \prime}=a^{\prime}$, we conclude that

$$
\left(\begin{array}{c}
a^{\prime} \\
a^{\prime}
\end{array}\right) \rightarrow\left(\begin{array}{c}
a^{\prime} \\
a^{\prime}
\end{array}\right)_{2}^{1,1}
$$

which contradicts Theorem 47 .

We may mention that there is an obvious extension of Theorem 51 to relations

$$
\left(\begin{array}{l}
a_{0} \\
\vdots \\
a_{t-1}
\end{array}\right) \rightarrow\left(\begin{array}{l}
a_{0} \\
\vdots \\
a_{t-1}
\end{array}\right)
$$

for any $t$.

In conclusion, we collect some polarised partition relations involving the first three infinite cardinals. They follow from Theorems 47-50. We put $\boldsymbol{\aleph}_{0}=a ; \boldsymbol{\aleph}_{1}=b ; \boldsymbol{\aleph}_{2}=d$.

$$
\begin{gathered}
\left(\begin{array}{l}
a \\
a
\end{array}\right) \rightarrow\left(\begin{array}{ll}
a & 1 \\
1 & a
\end{array}\right)^{1,1} ;\left(\begin{array}{l}
b \\
b
\end{array}\right) \rightarrow\left(\begin{array}{ll}
b & 1 \\
1 & b
\end{array}\right)^{1,1} ; \\
\left(\begin{array}{l}
d \\
d
\end{array}\right)+\left(\begin{array}{ll}
d & 1 \\
1 & d
\end{array}\right)^{1,1} \\
\left(\begin{array}{l}
a \\
b
\end{array}\right) \rightarrow\left(\begin{array}{ll}
a & a \\
a & b
\end{array}\right)^{1,1} ;\left(\begin{array}{l}
a \\
d
\end{array}\right) \rightarrow\left(\begin{array}{ll}
a & a \\
a & d
\end{array}\right)^{1,1}
\end{gathered}
$$

(Theorem 47);

(Theorem 48). 
If $2^{a}=b$, then

$$
\left(\begin{array}{l}
a \\
d
\end{array}\right) \rightarrow\left(\begin{array}{ll}
a & a \\
d & d
\end{array}\right)^{1,1}
$$

and

$$
\left(\begin{array}{l}
a \\
b
\end{array}\right) \rightarrow\left(\begin{array}{ll}
a & a \\
b & b
\end{array}\right)^{1,1}
$$

It seems curious that the continuum hypothesis should enable us both to strengthen

$$
\left(\begin{array}{l}
a \\
d
\end{array}\right) \rightarrow\left(\begin{array}{ll}
a & a \\
a & d
\end{array}\right)^{1,1}
$$

to

$$
\left(\begin{array}{l}
a \\
d
\end{array}\right) \rightarrow\left(\begin{array}{ll}
a & a \\
d & d
\end{array}\right)^{1,1}
$$

and to show that

$$
\left(\begin{array}{l}
a \\
b
\end{array}\right) \rightarrow\left(\begin{array}{ll}
a & a \\
a & b
\end{array}\right)^{1,1}
$$

cannot be strengthened to

$$
\left(\begin{array}{l}
a \\
b
\end{array}\right) \rightarrow\left(\begin{array}{ll}
a & a \\
b & b
\end{array}\right)^{1,1}
$$

\section{REFERENCES}

1. F. Bagemihl and H. D. Sprinkle, On a proposition of Sierpinski, Proc. Amer. Math. Soc. vol. 5 (1954) pp. 726-728.

2. B. Dushnik and E. W. Miller, Partially ordered sets, Amer. J. Math. vol. 63 (1941) p. 605.

3. P. Erdös, Some set-theoretical properties of graphs, Revista Universidad Nacional de Tucuman, Serie A vol. 3 (1942) pp. 363-367.

4. P. Erdös and R. Rado, A combinatorial theorem, J. London Math. Soc. vol. 25 (1950) pp. 249-255.

5. Combinatorial theorems on classifications of subsets of a given set, Proc. London Math. Soc. (3) vol. 2 (1952) pp. 417-439.

6. - A problem on ordered sets, J. London Math. Soc. vol 28 (1953) pp. 426-438.

7. P. Erdös and G. Szekeres, A combinatorial problem in geometry, Compositio Math. vol. 2 (1935) pp. 463-470.

8. P. Hall, On representations of sub-sets, J. London Math. Soc. vol. 10 (1934) pp. $26-30$. 
9. F. Hausdorff, Grundzilge einer theorie der geordneten Mengen, Math. Ann. vol. 65 (1908) pp. 435-506.

10. —, Mengenlehre, 3d ed., 1944, \$16.

11. R. Rado, Direct decompositions of partitions, J. London Math. Soc. vol. 29 (1954), pp. 71-83.

12. F. P. Ramsey, On a problem of formal logic, Proc. London Math. Soc. (2) vol. 30 (1930) pp. 264-286.

13. W. Sierpinski, Lȩ̧ons sur les nombres transfinis, Paris, 1928.

14. - $O$ jednom problemu $G$ Ruzjevica koji se odnosi na hipotezu kontinuuma, Glas Srpske Kraljevske Akademije vol. 152 (1932) pp. 163-169.

15. - Sur un problème de la théorie des relations, Annali R. Scuola Normale Superiore de Pisa Ser. 2 vol. 2 (1933) pp. 285-287.

16. - Concernant l'hypothèse du continu, Académie Royale Serbe. Bulletin de l'Académie des Sciences Mathématiques et Naturelles. A. Sciences Mathématiques et Physiques vol. 1 (1933) pp. 67-73.

17. A. Tarski, Quelques theorèmes sur les alephs, Fund. Math. vol. 7 (1925) p. 2.

HeBrew UNIVERSity OF JeRUSAlEM AND

UNIVERSITY OF READING 